
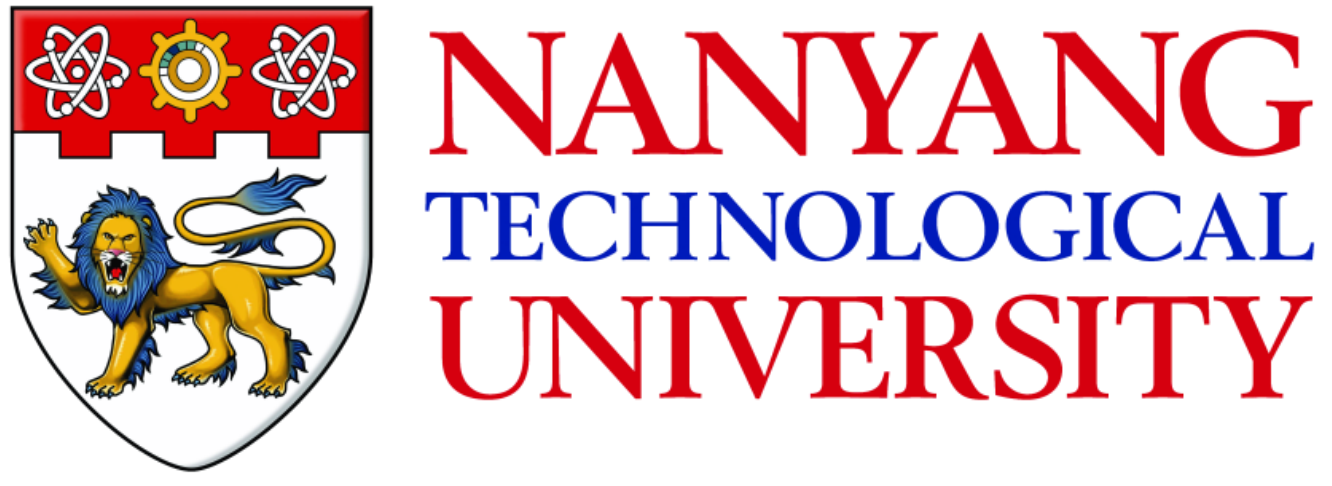

THE MOLECULAR DYNAMICS SIMULATION STUDIES OF NANOSCALE MECHANICAL DEFORMATION BEHAVIORS AND MECHANISMS IN METALLIC GLASSES

TANG CHAO

SCHOOL OF MECHANICAL AND AEROSPACE ENGINEERING 2016 



\section{THE MOLECULAR DYNAMICS SIMULATION STUDIES OF NANOSCALE MECHANICAL DEFORMATION BEHAVIORS AND MECHANISMS IN METALLIC GLASSES}

TANG CHAO

School of Mechanical and Aerospace Engineering A thesis submitted to the Nanyang Technological University in partial fulfilment of the requirement for the degree of Doctor of Philosophy 



\section{ACKNOWLEDGEMENT}

I would like to express my sincere gratitude to my supervisor, Associate Professor Wong Chee How, for his continuous support and helpful guidance throughout my Ph.D. studies. He has exposed me to a broad range of interesting research topics as well as the Singaporean culture. His enthusiasms and knowledge in academic research have provided me invaluable input for my research project. His friendliness and personality also helped me to overcome the difficulties in both my research work and personal life.

I wish to thank our group members for their personal help and suggestions during my studies at Nanyang Technological University.

I like to thank all my friends for making my life enjoyable and memorable in Singapore.

Finally, I want to give my special thanks to my parents, Mrs. Wu Junrong and Mr. Tang Jiaxin, for their unconditional love, understanding, encouragement, and support over the years. They are always around whenever I need them and offered me precious advice for my life. 


\section{TABLE OF CONTENTS}

ACKNOWLEDGEMENT ...............................................................................................

TABLE OF CONTENTS .................................................................................................. iii

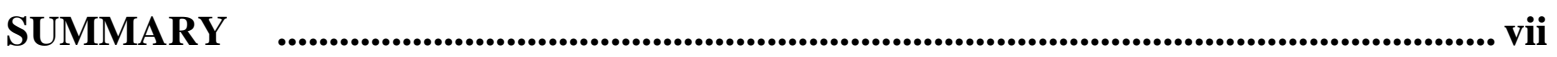

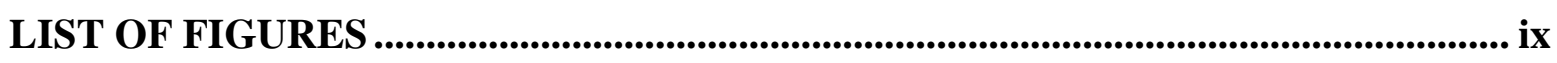

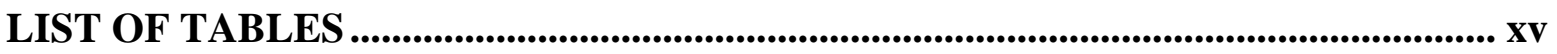

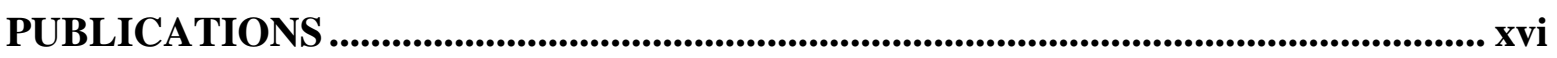

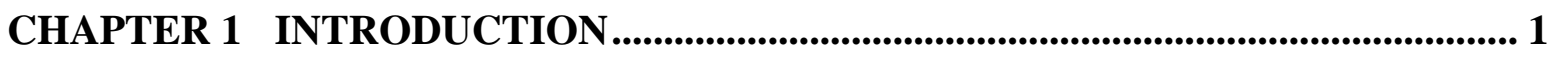

1.1 Formation and history of metallic glasses.............................................

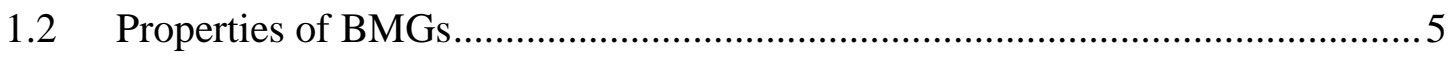

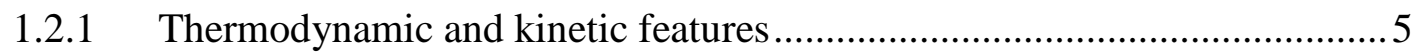

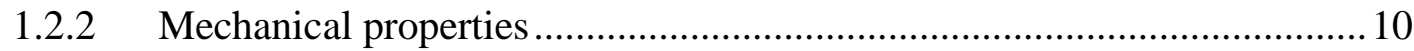

$1.3 \quad$ Microscopic structure of metallic glasses ..................................................... 14

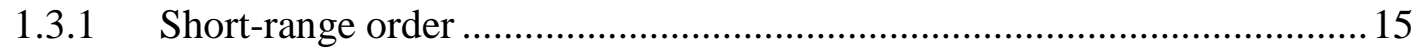

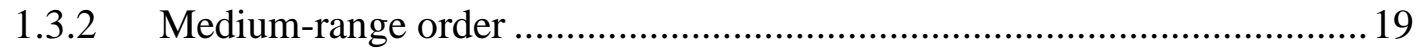

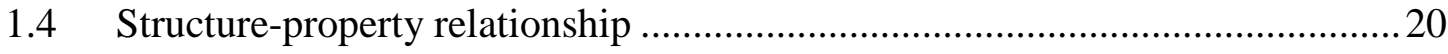

1.4.1 Structural-thermodynamic relationship ...........................................20

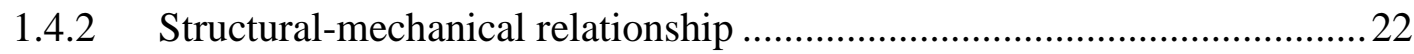

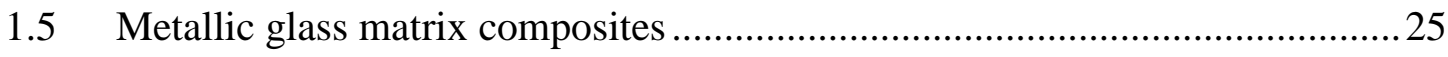

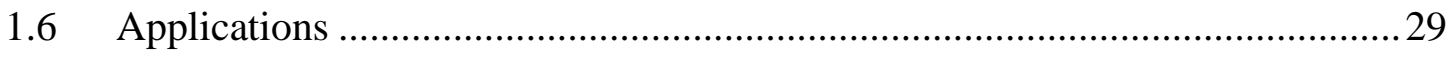




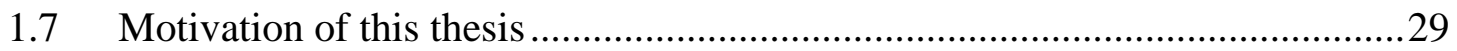

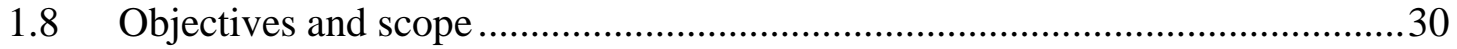

CHAPTER 2 CLASSICAL MOLECULAR DYNAMICS........................................... 33

$2.1 \quad$ Applications of molecular dynamics .............................................................33

2.2 Main features of molecular dynamics simulation ...........................................34

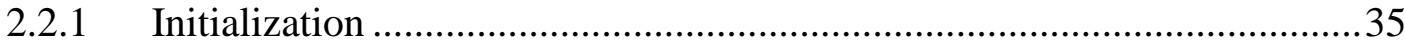

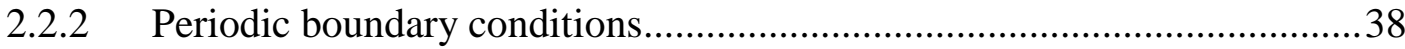

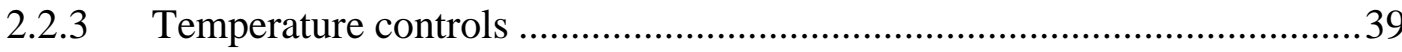

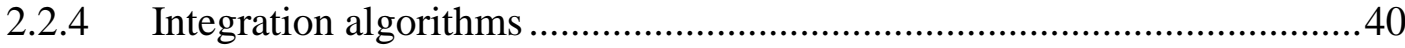

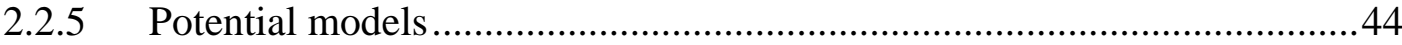

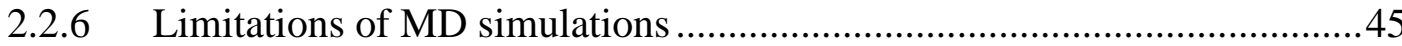

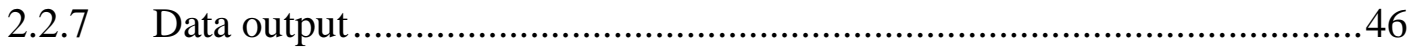

CHAPTER 3 SHORT-RANGE ORDER AND DEFECTS IN METALLIC GLASSES STUDIED BY ATOMIC LEVEL STRESS THEORY .............................. 47

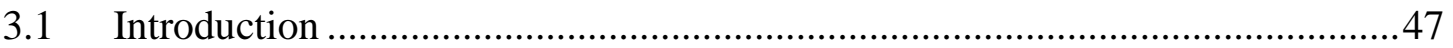

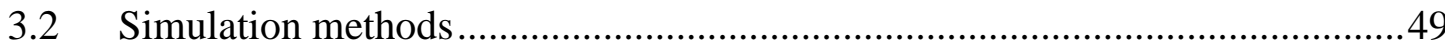

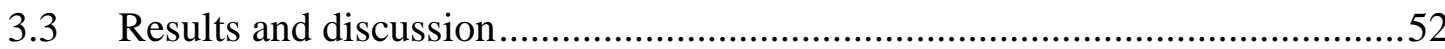

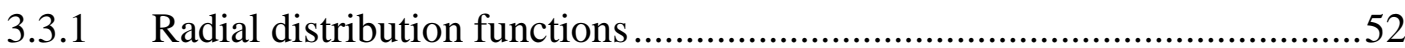

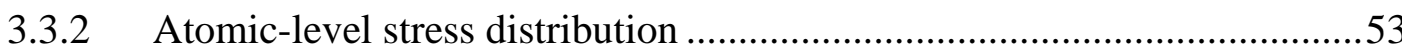

3.3.3 Influences of atomic shear stress on local dynamics ..............................55

3.3.4 Influence of atomic shear stress on local mechanical responses ...............58

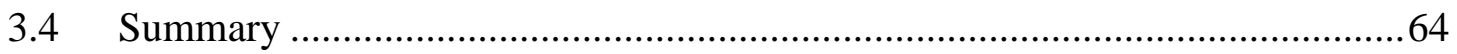


CHAPTER 4 SOLID-LIKE AND LIQUID-LIKE NETWORKS IN ZR-CU-AL METALLIC GLASS.

4.1 Introduction .65

4.2 Simulation methods 68

4.2.1 Computational models .68

4.2.2 Calculation of nearest-neighbor correlation index .68

4.3 Results and discussion .71

4.3.1 Solid-like and liquid-like networks on the medium-range scale .71

4.3.2 Influences of atomic level stress on local topology .77

4.3.3 Mechanical discrepancies between solid-like and liquid-like regions.......81

4.4 Summary .85

\section{CHAPTER 5 CHEMICAL SHORT RANGE ORDER AND ITS} CORRELATIONS WITH DYNAMIC/MECHANICAL HETEROGENEITIES IN ZR-CU-AG METALLIC GLASS.

5.1 Introduction .86

5.2 Simulation methods. .88

5.2.1 Computational models 88

5.2.2 Chemical short range order parameter .89

5.3 Results and discussion .89

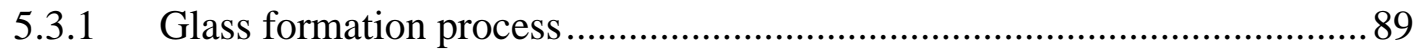

5.3.2 Formation of chemical short range order............................................. 92

5.3.3 Relationships between chemical and dynamic heterogeneities .97 
5.4 Summary

CHAPTER 6 CONCLUSIONS AND FUTURE WORK

6.1 Conclusions 103

6.2 Main contributions arising from this thesis 105

6.3 Future work 105

6.3.1 Crystal-like order in multicomponent glasses. 106

6.3.2 Various factors affecting the mechanical behaviors of metallic glass matrix nanocomposites 107 


\section{SUMMARY}

As a new class of materials, metallic glasses (MGs) appear to have great potential for engineering applications, due to their superior properties including high strength, good corrosion resistance, thermoformability and etc. Despite several attractive traits of MGs, the main drawback lies in their little tensile and limited compressive plasticity at low temperature. Typically, the topological and chemical short-to-medium range structure is believed to be intimately linked with the physical and mechanical behaviors of MGs, and hence becomes one of the fundamental interests for many researchers. With this regard, the main objective of this $\mathrm{PhD}$ project is to examine the structure-property relationships in MGs by using molecular dynamics (MD) simulations.

During the past decades, many simulation studies have been conducted to investigate the intrinsic structural defects in MGs, such as free volume, atomic-level stress, Voronoi polyhedron and etc. For instance, full icosahedra with a Voronoi index of $\langle 0,0,12,0\rangle$ are found to exhibit ultrahigh thermodynamic and mechanical stability in $\mathrm{Cu}_{64} \mathrm{Zr}_{36} \mathrm{MG}$. By modeling annealing and shear deformation processes, the effects of atomic-level stress on local dynamic and mechanical properties of MGs were investigated. Other than Voronoi index, atomic-level stress is proven to be a generic parameter to identify the structural defects in different glasses. By modeling annealing processes around the glass transition temperature of MGs, atoms with high stress are found to exhibit faster dynamics than solid-like ones. In addition, atoms with excessive atomic shear stress serve as fertile sites for shear distortions, which may explain the shear localizations in MGs.

Despite numerous studies devoted to the short-range order in amorphous alloys, their medium-range structure remains a longstanding mystery. On the basis of Voronoi 
tessellation method, the correlations between local fivefold symmetry and mediumrange structures were investigated via MD simulations. It is shown that atoms with high and low degree of local fivefold symmetry (HLFFS and LLFFS) exhibit distinctly different characteristics in $\mathrm{Zr}_{46} \mathrm{Cu}_{46} \mathrm{Al}_{8}$ glasses. Both HLFFS and LLFFS atoms appear to connect with similar polyhedra, forming medium-range scale superclusters or networks. In addition, strong spatial avoidance between these two types of atoms is observed in the simulated models. Our quantitative analysis reveals the intimate correlations between atomic-level stress and short and medium-range structures in MGs. Moreover, the HLFFS and LLFFS networks respectively exhibit solid-like and liquidlike behaviors under shear loadings. The characteristic length of HLFFS and LLFFS networks appears to be consistent with the mechanical heterogeneity found in experiments.

The topological structures and their correlations with $\mathrm{MG}$ properties have been extensively studied via experiments and simulations. Nevertheless, further investigations concerning the effects of chemical short range order (CSRO) on the MG properties are still lacking. This thesis also discusses the CSRO development and its effects on the dynamic and mechanical heterogeneities in $\mathrm{Zr}_{45} \mathrm{Cu}_{45} \mathrm{Ag}_{10}$ glasses. Similar to the TSRO reported in previous studies, the CSRO becomes prominent in the supercooled region. The development of CSRO is directly attributed to the local energetic stability. Due to the positive enthalpy of mixing of $\mathrm{Cu}-\mathrm{Ag}$ pairs, nanoscale phase separation is formed during the quenching process, which is characterized by the $\mathrm{Cu}$-rich regions and $\mathrm{Ag}$-rich regions. In contrast to Ag-rich regions, the $\mathrm{Cu}$-rich regions exhibit higher dynamic and mechanical stability under external stimuli. The simulation results reveal that the dynamic and mechanical heterogeneities of MGs are also related with the phase separation phenomenon. 


\section{LIST OF FIGURES}

Figure 1.1 Correlations among critical cooling rates $\left(\mathrm{R}_{\mathrm{c}}\right)$, reduced glass temperature $\left(\mathrm{T}_{\mathrm{rg}}\right)$, and critical sample thickness $\left(\mathrm{t}_{\max }\right)$ for recently developed and conventional BMGs [17].

Figure 1.2 BMG systems with different maximum diameters and their discovered years 5

Figure 1.3 Increase of free energy difference $\Delta \mathrm{G}$ during the supercooling of BMGs with different GFA [37]. 7

Figure 1.4 Temperature influences on the viscosities of various glass-forming liquids

Figure 1.5 Schematics of the deformation mechanisms in amorphous alloys, including (a) a shear transformation zone [45], and (b) a local atomic rearrangement based on freevolume model [48].

Figure 1.6 Compressive and tensile stress-strain curves of $\mathrm{Zr}_{57} \mathrm{Nb}_{5} \mathrm{Cu}_{15.4} \mathrm{Ni}_{12.6} \mathrm{Al}_{10}$ amorphous alloy [58].

Figure 1.7 The fracture behaviors of $\mathrm{Pd}_{40} \mathrm{Ni}_{40} \mathrm{P}_{20}$ glasses under (a) tension and (b) compression, illustrating the difference in fracture angles [63].

Figure 1.8 Solute-centered clusters discovered in the simulated $\mathrm{Ni}_{81} \mathrm{P}_{19}$ amorphous alloy [64]. B and Ni atoms are colored purple and green, respectively. The MRO is formed by the interconnection of those quasiequivalent clusters

Figure 1.9 Theoretical coordination number of an element $i$ in liquid of element $j$ as a function of the radius ratio $\mathrm{R}^{*}=\mathrm{r}_{\mathrm{i}} / \mathrm{r}_{\mathrm{j}}[66]$

Figure $1.10 \mathrm{CN}$ distributions of the solute atoms in various MGs, based on ab initio calculations [64]. The average $\mathrm{CN}$ varies with the effective atomic radius ratio $\mathrm{R}^{*}$. 17 
Figure 1.11 Several Voronoi polyehedra with different CNs [12]. Clusters are formed by the centeral and neighboring atoms, which are colored cyan and blue, respectively. The corresponding Voronoi polyhedra are depicted by the yellow lines. .18

Figure 1.12 Distributions of atomic mobility (propensity for motion) of $\mathrm{Cu}$ atoms in $\mathrm{Cu}_{64} \mathrm{Zr}_{36}$ supercooled liquid (at $800 \mathrm{~K}$ ) [73]. Right and left sides denote the $5 \% \mathrm{Cu}$ atoms having the fastest and slowest mobility, respectively. In the inset, $\mathrm{Cu}$-centered polyhedra are sorted according to the atomic mobility (low to high) and then separated into 20 groups, each involving $5 \%$ of the total $\mathrm{Cu}$ atoms.

Figure 1.13 Relationship between icosahedral clusters and local inelastic deformations (STZ) [80]. Such figure displays the cross-section view of a sheared $\mathrm{Cu}_{64} \mathrm{Zr}_{36}$ sample. The configuration only depicts $\mathrm{Cu}$ atoms for clarity, indicating the $\mathrm{Cu}$-centered polyhedra. The coloring schemes are assigned according to the different values of von Mises shear strains. The elastically and plastically deformed and regions are colored dark purple and yellow respectively. The circles represent two elastic entities and one STZ, and their local structures are illustrated in the insets. The $\mathrm{Cu}$ atoms in the elastic entities are mostly in $\langle 0,0,12,0\rangle$ FI (such $\mathrm{Cu}$-centered polyhedra are mapped blue), while the $\mathrm{Cu}$ atoms in the STZ are mostly embedded in unfavorable (liquid-like) clusters (mapped red). The $\mathrm{Cu}$ atoms of other regular clusters are displayed by small green dots.

Figure 1.14 Comparison of the deformation behaviors in terms of shear localization in (a) $\mathrm{Cu}_{64} \mathrm{Zr}_{36}$ and (b) $\mathrm{Cu}_{40} \mathrm{Zr}_{60}$ glasses [89]. Only atoms with atomic shear strain higher than 0.3 are shown for clarity. .24

Figure 1.15 Scanning electron microscopy (SEM) image of the microstructure of the $\left(\mathrm{Zr}_{75} \mathrm{Ti}_{18.34} \mathrm{Nb}_{6.66}\right)_{75} \mathrm{X}_{25}$ in-situ composites [94]. .27

Figure 2.1 Flow chart representing the basic steps in MD simulations. 37 
Figure 2.2 Schematic of the periodic boundary conditions adopted in a two dimensional system. The central cube represents the primary cell, and the surrounding ones are replicated images. Particles can move across the boundaries of each cube 38

Figure 3.1 (a) 3D Configuration of the simulated $\mathrm{Cu}_{50} \mathrm{Zr}_{50}$ MG. (b) Schematic of shear loading on a $\mathrm{Cu}_{50} \mathrm{Zr}_{50} \mathrm{MG}$ by using supercell tilting. .50

Figure 3.2 Comparison between RDFs obtained by experiment [131] and MD simulation in three different compositions of $\mathrm{Cu}_{\mathrm{x}} \mathrm{Zr}_{100-\mathrm{x}}$ MGs. Individual curves are shifted vertically for clarity.

Figure 3.3 Distribution of von Mises shear stress multiplied by atomic volume in $\mathrm{Cu}_{50} \mathrm{Zr}_{50} \mathrm{MG}$ .54

Figure 3.4 Correlations between $\gamma$-defects and atomic mobility in $\mathrm{Cu}_{\mathrm{x}} \mathrm{Zr}_{100-\mathrm{x}}$ glass forming liquids. $\mathrm{Cu}$ and $\mathrm{Zr}$ atoms are sorted by their displacement from low to high. Atoms are then divided into 10 groups, each containing $10 \%$ of the total $\mathrm{Cu}$ or $\mathrm{Zr}$ atoms. The left and right end represent the $10 \%$ atoms with the lowest and highest mobility, respectively. The histogram shows the fraction of $\gamma$-defects in each group. 57

Figure 3.5 The average atomic shear strain evolutions of $\gamma$-defects and solid-like atoms for (a) $\mathrm{Cu}$ and (b) $\mathrm{Zr}$ atoms during shear deformation in three different compositions. Individual curves are shifted horizontally for clarity 60

Figure 3.6 The PRSTZ evolutions of $\gamma$-defects and solid-like atoms for (a) $\mathrm{Cu}$ and (b) $\mathrm{Zr}$ atoms during shear deformation in $\mathrm{Cu}_{50} \mathrm{Zr}_{50} \mathrm{MG}$. 62 Figure 4.1 Fractions of the most populated Voronoi polyhedra in $\mathrm{Zr}_{46} \mathrm{Cu}_{46} \mathrm{Al}_{8} \mathrm{MG}_{\text {....... }} 70$ Figure 4.2 Correlation matrix of different coordination polyhedra in $\mathrm{Zr}_{46} \mathrm{Cu}_{46} \mathrm{Al}_{8} \mathrm{MG}$. The colors represent the correlation strength (see text for details). .72 
Figure 4.3 Correlation matrix of atoms with difference degree of local fivefold symmetry in (a) $\mathrm{Cu}_{46} \mathrm{Zr}_{46} \mathrm{Al}_{8}$ and (b) $\mathrm{Mg}_{65} \mathrm{Cu}_{25} \mathrm{Y}_{10}$ glass-forming liquids at $\mathrm{T}=1.2 \mathrm{~T}_{\mathrm{g}}$ [159]. (The correlation maps exhibit similar features at different temperatures). .74 Figure 4.4 (a) Spatial distributions and connections of HLFFS and LLFFS atoms in the $\mathrm{Zr}_{46} \mathrm{Cu}_{46} \mathrm{Al}_{8} \mathrm{MG}$ within a thickness of $6 \AA$. Only HLFFS and LLFFS atoms are displayed for clarity. For the spatial heterogeneity in glass, HLFFS atoms (colored green) are percolated and form networks, and LLFFS atoms (colored red) have the similar tendency. The connectivity between HLFFS and LLFFS atoms is weak. (b) 3D configurations of two networks respectively formed by HLFFS and LLFFS atoms. The $\mathrm{Cu}, \mathrm{Zr}$ and $\mathrm{Al}$ atoms are colored purple, red and yellow, respectively. .76 Figure 4.5 Distribution of atomic shear stress multiplied by atomic volume in $\mathrm{Zr}_{46} \mathrm{Cu}_{46} \mathrm{Al}_{8} \mathrm{MG} . \sigma_{i} \cdot v_{i}$ could be directly extracted from the MD simulation; to further calculate the atomic level shear stress, the atomic volume for each atom should be defined (or use the average atomic volume). .77

Figure 4.6 Spatial distribution of $\mathrm{Cu}$ atoms with excessive positive/negative atomic stress in terms of (a) $\sigma_{i}^{x x}$ and (b) $\tau_{i}^{x y}$ within a thickness of $6 \AA$, to illustrate the dependence of atomic level stress on spatial heterogeneity in MGs. Only atoms with excessive stress are displayed for clarity. Atoms with excessive positive stress (colored red) are heterogeneously distributed and tend to form percolated networks, and atoms with excessive negative stress (colored blue) have the similar trend, indicating atoms with similar stress are likely to be percolated. .80

Figure 4.7 Overall stress-strain curve of $\mathrm{Zr}_{46} \mathrm{Cu}_{46} \mathrm{Al}_{8} \mathrm{MG}$ under pure shear loading. ....81 Figure 4.8 The average atomic shear strain evolution of HLFFS and LLFFS atoms for (a) $\mathrm{Cu}$, (b) $\mathrm{Zr}$ and (c) Al versus overall shear strain during the pure shear deformation of $\mathrm{Zr}_{46} \mathrm{Cu}_{46} \mathrm{Al}_{8} \mathrm{MG}$. .83 
Figure 4.9 Correlations between local plastic deformations and HLFFS and LLFFS atoms. This figure displays the cross-section view of a deformed $\mathrm{Zr}_{46} \mathrm{Cu}_{46} \mathrm{Al}_{8}$ sample, at shear strain of $10 \%$. The colors are mapped to the various levels of von Mises shear strains. The dark blue regions are elastically deformed while the green ones have participated in plastic deformations. Two elastic and two plastic regions are circled out and their local structures are illustrated in the insets. For clarity, only HLFFS and LLFFS atoms are shown in the insets. For the elastic regions, HLFFS atoms (colored green) are dominant and few LLFFS atoms (colored red) are observed. While in the plastically deformed regions, LLFFS atoms are abundant and HLFFS atoms are rarely found. .84

Figure 5.1 Temperature dependence of potential energy in $\mathrm{Zr}_{45} \mathrm{Cu}_{45} \mathrm{Ag}_{10}$ alloy during cooling from $2000 \mathrm{~K}$ to $50 \mathrm{~K}$. 3D configuration of the $\mathrm{Zr}_{45} \mathrm{Cu}_{45} \mathrm{Ag}_{10} \mathrm{MG}$ sample is shown in the inset. $\mathrm{Zr}, \mathrm{Cu}$ and $\mathrm{Ag}$ atoms are colored red, purple and yellow respectively.

Figure 5.2 Total and partial RDFs of $\mathrm{Zr}_{45} \mathrm{Cu}_{45} \mathrm{Ag}_{10} \mathrm{MG}$.

Figure 5.3 Temperature dependence of Warren-Cowley parameters for different pairs during the cooling process of $\mathrm{Zr}_{45} \mathrm{Cu}_{45} \mathrm{Ag}_{10}$ alloy. $\mathrm{Zr}-(\mathrm{Cu} / \mathrm{Zr} / \mathrm{Ag})$ pairs are omitted for clarity. .95

Figure 5.4 The dependence of atomic mobility ( $\mathrm{Zr}, \mathrm{Cu}$ and $\mathrm{Ag}$ atoms) on neighboring chemical compositions, showing the relationship between chemical and dynamic heterogeneities. Atoms in Ag-rich or $\mathrm{Cu}$-poor regions are mostly observed to exhibit faster dynamics. .98

Figure 5.5 The dependence of local shear resistance $(\mathrm{Zr}, \mathrm{Cu}$ and $\mathrm{Ag}$ atoms) on neighboring chemical compositions, showing the relationship between chemical and 
mechanical heterogeneities. Similarly, atoms in Ag-rich or Cu-poor regions exhibit lower resistance to shear deformations. 


\section{LIST OF TABLES}

Table 1.1 Correlations between selected properties and the fragility of the supercooled liquids in glass forming systems [39]. 9

Table 4.1 The average atomic shear stress (multiplied by atomic volume) for different Voronoi polyhedra in $\mathrm{Zr}_{46} \mathrm{Cu}_{46} \mathrm{Al}_{8} \mathrm{MG}$. 78

Table 4.2 Spatial correlation index $C_{i j}$ of atoms with highest/lowest atomic shear stress.

Table 5.1 The interatomic distances $(\AA)$ for different pairs in $\mathrm{Zr}_{45} \mathrm{Cu}_{45} \mathrm{Ag}_{10} \mathrm{MG}$ 92

Table 5.2 Partial and total coordination number for different elements in $\mathrm{Zr}_{45} \mathrm{Cu}_{45} \mathrm{Ag}_{10}$ MG. .92

Table 5.3 Comparison of Warren-Cowley parameters at $2000 \mathrm{~K}$ and $50 \mathrm{~K}$ in $\mathrm{Zr}_{45} \mathrm{Cu}_{45} \mathrm{Ag}_{10} \mathrm{MGs}$ obtained at different cooling rates

Table 5.4 Average potential energies of central $\mathrm{Cu}$ and $\mathrm{Ag}$ atoms with different neighboring compositions at $50 \mathrm{~K}$ 95 


\section{PUBLICATIONS}

The following SCI journal publications are related to the present research:

1. C. Tang and C. H. Wong, "Effect of atomic-level stresses on local dynamic and mechanical properties in $C u_{x} Z r_{100-x}$ metallic glasses: A molecular dynamics study," Intermetallics 58, pp. 50-55, 2015.

2. C. Tang and C. H. Wong, "A molecular dynamics simulation study of solid-like and liquid-like networks in Zr46Cu46Al8 metallic glass," Journal of Non-Crystalline Solids 422, pp. 39-45, 2015.

3. C. Tang and C. H. Wong, "Formation of chemical short range order and its influences on the dynamic/mechanical heterogeneity in amorphous $\mathrm{Zr}-\mathrm{Cu}$-Ag alloys: A molecular dynamics study," Intermetallics 70, pp. 61-67, 2016. 


\section{CHAPTER 1 INTRODUCTION}

Metals are conventionally made of metallic bondings and considered to possess translational ordering on the macroscopic scale. Glasses, on the other hand, often consist of covalent and ionic bonds, and are characterized by their amorphous structure lacking geometrical symmetry. Due to the different atomic and electronic structure, these two classes of materials have been realized to exhibit distinctly different attributes. In the 1960s Klement et al. [1] successfully synthesized Au-Si metallic glass (MG), characterized by the non-crystalline structure without long-range ordering. The combination of metallic nature and amorphous structure in MGs leads to the unique properties and extensive potential for commercial applications [2, 3]. The discovery of first MG has aroused remarkable attention in exploring and understanding this young class of materials [4-9]. The so-called "bulk" metallic glasses (BMGs) represent the impressive progress in this endeavor, with sample size or thickness in excess of $1 \mathrm{~mm}$. These bulk glass formers, generally complex alloys with multicomponent elements, can be readily synthesized into fully amorphous samples with slow cooling rates.

One of the enduring fascinations for scientists and engineers is the intriguing physical, chemical and mechanical properties of MGs. Due to the absence of dislocation defects, most MGs possess higher strengths and elastic limits compared to conventional metals, along with good wear and corrosion resistance [8, 10]. However, BMGs typically undergo highly localized deformation by the initialization and propagation of shear bands at ambient temperature, leading to their catastrophic failure with limited compressive ductility and negligible tensile ductility [11]. Additionally, one of the most important characteristics of BMGs is the glass transition behavior, which represents the transformation from undercooled melts into glassy state when cooled from high to low 
temperature [12]. The interesting properties of BMGs have inspired fundamental research interest on the glass-forming ability (GFA), atomic-level structure, structureproperty relationship and deformation mechanisms. The discovery of BMG samples and their unique attributes have made them attractive and promising in many commercial endeavors, including aerospace, naval, sports equipment, micro electromechanical systems (MEMs), nanotechnology and biomedical devices.

In addition to experimental techniques, computer simulations have been extensively adopted as an alternative to explore the structural and mechanical behaviors of BMGs. Therefore in this work, molecular dynamics (MD) simulations are implemented to investigate the microscopic properties of MGs.

\subsection{Formation and history of metallic glasses}

Metals have been known to possess a crystalline structure for a long time. In the 1960s, the discovery of first $\mathrm{MG}, \mathrm{Au}_{75} \mathrm{Si}_{25}$, was announced by Klement et al. in the California Institute of Technology [1]. The so-called MG is an alloy that maintains the amorphous structure of high-temperature melts. By using rapid quenching technique at sufficiently high cooling rates of $10^{5}-10^{6} \mathrm{~K} / \mathrm{s}$, the crystal nucleation and growth can be kinetically suppressed in some alloy melts, forming frozen liquids with amorphous structure [13]. The fabrication, structure and properties of MGs have attracted intense attention due to their scientific significance and potential for engineering applications. Over the next decade, commercial manufacture of low dimensional metallic glass, such as ribbons, lines and sheets, has been extensively studied for the use of soft magnetic materials [14]. Most samples were synthesized with limited thickness to ensure that the heat in the metallic liquid could be drawn off quickly enough, such that the amorphous structures can be retained during the solidification. However, the extended applications of MGs were restricted because of the requirement of extremely high critical cooling rates. 
Meanwhile, Turbull and coworkers proposed a crucial criterion to discover alloys with excellent GFA $[15,16]$. The reduced glass transition temperature, $T_{r g}=T_{g} / T_{m}\left(T_{m}\right.$ and $T_{g}$ respectively denote the melting and glass transition temperature) was proposed to predict the GFA of MGs. According to such standard, a liquid with $T_{r g}=0.67$ becomes very sluggish in crystallization and hence can be readily undercooled into bulk glassy sample at a slow quenching rate. The Turbull criterion has play an essential role in finding various MGs with excellent GFA. The correlations between maximum samples thickness $\left(t_{\max }\right)$, critical cooling rates $\left(R_{c}\right)$ and reduced glass transition temperature $\left(T_{r g}\right)$ for recently developed and traditional MGs was illustrated in Figure 1.1. Clearly, the GFA tends to increase with increasing reduced glass transition temperature, indicating the suitability of the Turbull criterion.

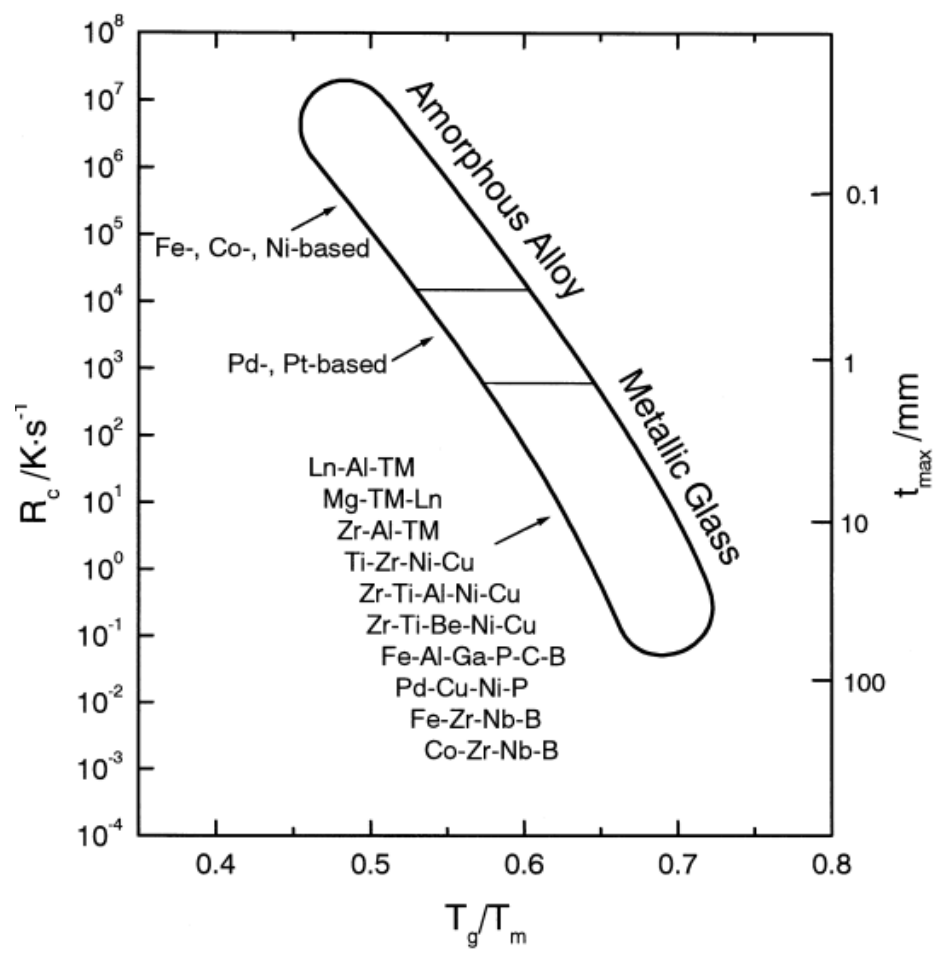

Figure 1.1 Correlations among critical cooling rates $\left(R_{c}\right)$, reduced glass temperature $\left(T_{r g}\right)$, and critical sample thickness $\left(t_{\max }\right)$ for recently developed and conventional BMGs [17]. 
One can arbitrarily define the millimeter size as bulk. Chen discovered the first BMG (Pd-Cu-Si glass) in the 1970s [18]. With simple suction-casting techniques, the Pd-CuSi alloy can be cast into a cylindrical sample with millimeter-diameter and at a critical quenching rate of $10^{3} \mathrm{~K} / \mathrm{s}$. In 1982, a well-known BMG was successfully prepared with a composition of $\mathrm{Pd}_{40} \mathrm{Ni}_{40} \mathrm{Si}_{20}$, and the sample was slowly quenched $(1.4 \mathrm{~K} / \mathrm{s})$ by adopting boron oxide fluxing method [19]. Unfortunately, due to the expensive cost of Pd metal, the interests for Pd-based BMGs were only limited to research laboratories. In the late 1980s, Inoue et al. [20] has made a breakthrough in finding many complex BMG systems with common metallic elements. For instance, exceptional GFA has been observed in the rare-earth-based systems, such as La-Al-Cu and La-Al-Ni alloys. Over the next decade, similar quaternary and quinary BMGs has been developed at quenching speed below $100 \mathrm{~K} / \mathrm{s}$ and the critical size is up to several centimeters [21, 22]. In the early 1990s, Zr-based alloys were fabricated into BMG samples with a diameter up to 16 mm [23]. In 1997, the Pd-Cu-Ni-P system with extraordinary GFA and critical diameter of $72 \mathrm{~mm}$ was developed by Inoue et al. [24]. Another excellent BMG with a composition of $\mathrm{Zr}_{41.2} \mathrm{Ti}_{13.8} \mathrm{Cu}_{12.5} \mathrm{Ni}_{10.0} \mathrm{Be}_{22.5}$, typically referred to as Vitreloy 1 (Vit 1), and critical quenching rate below $10 \mathrm{~K} / \mathrm{s}$ was reported by Johnson and coworkers [25]. Other excellent glass formers were soon discovered in the nickel-, copper-, iron- and titanium-based alloy systems [26-29]. Until now, the maximum achievable thickness of MG is around $80 \mathrm{~mm}$ in diameter [30]. Some BMGs with critical diameters in excess of $10 \mathrm{~mm}$ are exhibited in Figure 1.2. 


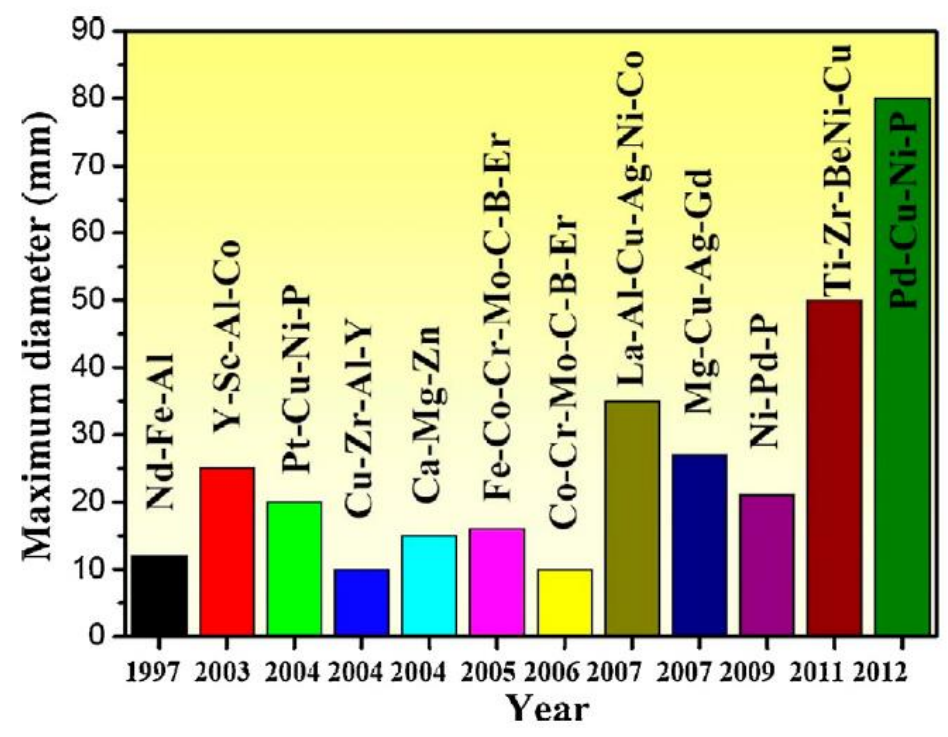

Figure 1.2 BMG systems with different maximum diameters and their discovered years [31].

Since the discovery of MGs, various empirical rules for BMG formation have been suggested to include: (1) multicomponent alloys consisting of at least three elements with complexity, and hence the energetic stability of a regular structure is reduced; (2) atomic size mismatch between constituent elements resulting in higher packing density; (3) alloy composition near deep eutectic, indicating a stable liquid at low temperature that can be easily quenched into glass; and (4) negative enthalpy of mixing between the constituents, which elevates the energy barrier at the liquid-solid interface and thus reducing the crystal nucleation rate [3].

\subsection{Properties of BMGs}

\subsubsection{Thermodynamic and kinetic features}

The good GFA of BMG forming liquids is mainly attributed to their slow kinetics [3234]. Recent studies found that the suitable choices of constituent elements would lead to MGs with excellent GFA. As well known, the GFA of alloys tend to increase with an increasing number of component elements. This behavior is called "confusion principle", 
indicating that the large number of differently atom sized elements can destabilize the competing crystalline phases during cooling [35].

In metallic systems, crystals are thermodynamic equilibrium states when the temperature is below $T_{m}$. The metallic liquids could be supercooled without crystallization by using high cooling rates and removing the heterogeneous nucleation sites. The supercooled liquid is considered "quasi-equilibrium" because it is not really in thermodynamically equilibrium state, even though the supercooled state can exist for a long time scale as it is difficult to crystallize at low temperature [12]. Generally speaking, the GFA of an alloy is equivalent to the ability to suppressing crystallization with the supercooled liquid. When the temperature is decreased to below $T_{m}$, the difference between the free energy of the supercooled liquid and the competing crystal, $\Delta G$, represents the driving force for crystallization. Interestingly, it has been found that the GFA scales inversely with $\Delta G$, which can be calculated as [13]

$$
\Delta G_{1-s}(T)=\Delta H_{f}-\Delta S_{f} T_{0}-\int_{T}^{T_{0}} \Delta C_{p}^{1-s}(T) d T+\int_{T}^{T_{0}} \frac{\Delta C_{p}^{1-s}(T)}{T} d T
$$

where $\Delta H_{f}$ and $\Delta S_{f}$ refer to the enthalpy and entropy of fusion respectively, $T_{0}$ is the temperature at which the crystal and the liquid are in equilibrium, and $\Delta C_{p}(T)$ is the specific heat capacity difference. Therefore, a low $\Delta G$ represents a small enthalpy of fusion $\Delta H_{f}$ and large entropy of fusion $\Delta S_{f}$. The large entropy is typically associated with multicomponent systems, since $\Delta S_{f}$ scales with the number of microscopic states [36]. Also at a constant temperature, the free energy difference decreases with lower enthalpy and higher value of $T_{r g}$, which agrees with "confusion principle" and empirical rules mentioned above. Figure 1.3 illustrates the driving force in several BMG systems with different GFA (critical cooling rates). As shown in the figure, the GFA is strongly 
coupled to the free energy difference. In addition, the driving force increases during supercooling, as the supercooled liquid gradually deviates from the equilibrium state (crystal).

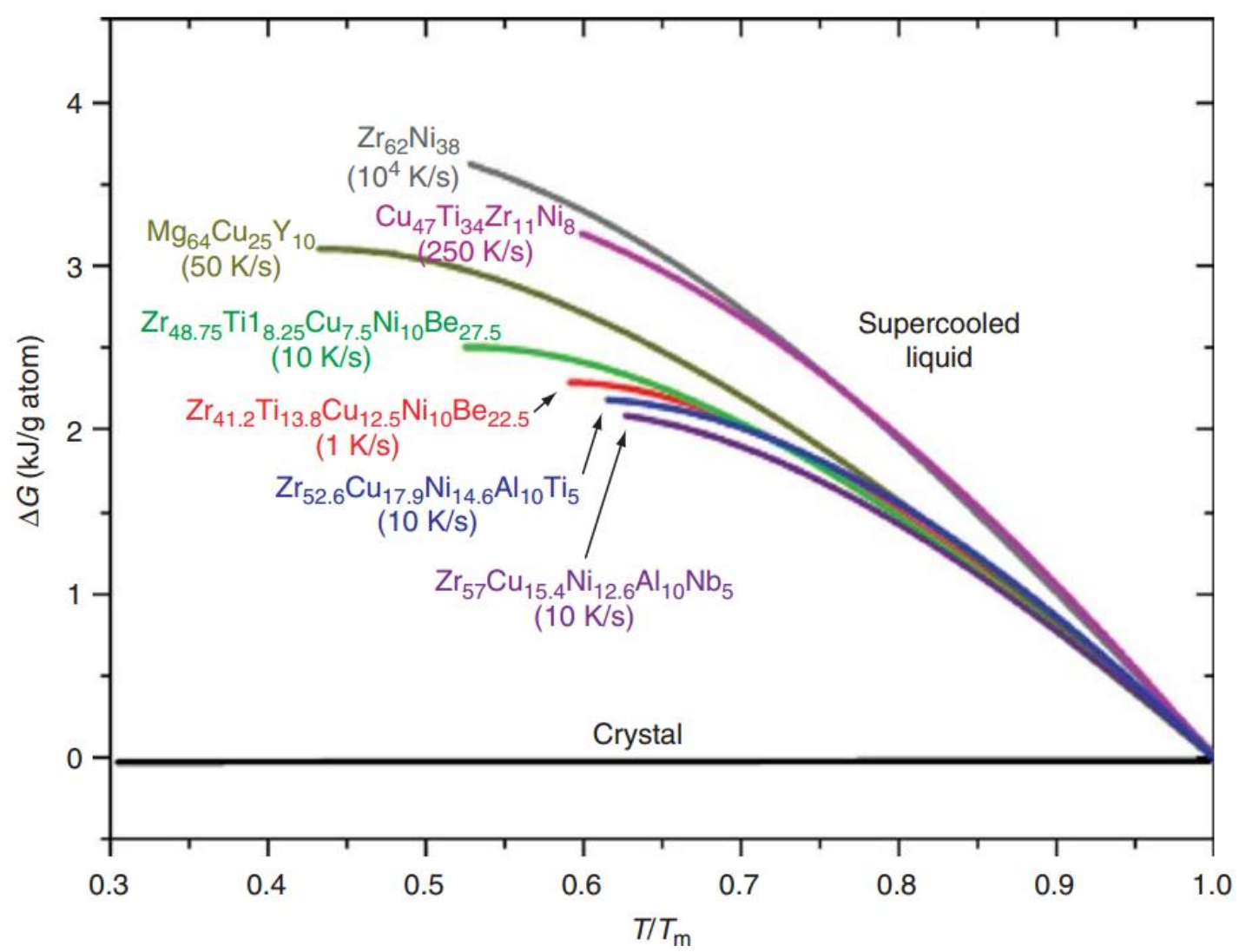

Figure 1.3 Increase of free energy difference $\Delta G$ during the supercooling of BMGs with different GFA [37]. 


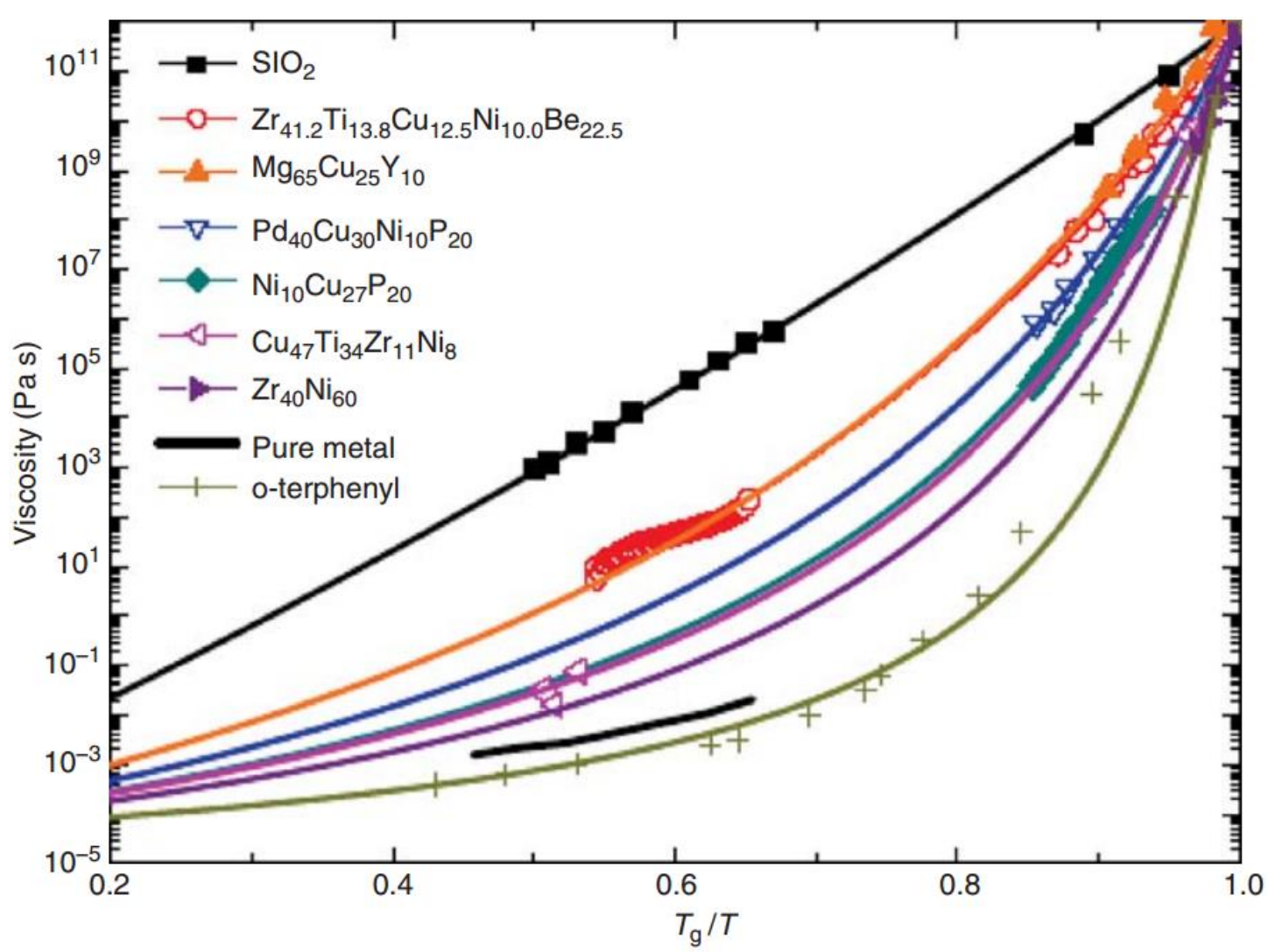

Figure 1.4 Temperature influences on the viscosities of various glass-forming liquids [37].

To get a better understanding of the GFA in BMGs, one needs to study the kinetic properties in glass forming systems. The viscosity $\eta$ plays a vital role on the GFA of a glass-forming system, and also reflects the mobility of atoms during supercooling. The viscosity can be directly measured in BMGs and supercooled liquids in a wide time and temperature scales, as many BMGs are relatively stable towards crystallization on laboratory time scales. Figure 1.4 shows the correlations between viscosity and temperature in various glasses, and the viscosity data shows good agreement with the Vogel-Fulcher-Tammann (VFT) equation [38] such that

$$
\eta=\eta_{0} \exp \left[\frac{D T_{v}}{T-T_{v}}\right]
$$


where $T_{v}$ is the Vogel-Fulcher temperature, at which the flow resistance goes to infinity. $D$ is recognized as the fragility parameter, which is associated with the GFA of MGs. The glass transition temperature is commonly defined as the temperature at which the viscosity exceeds a critical value (e.g., $\left.10^{12} \mathrm{~Pa} \cdot \mathrm{s}\right)$, and the slope at $T_{g}$ denotes the fragility [39]. Liquids with larger fragility are termed "fragile", as they exhibit an abrupt change of viscosity around glass transition. On the other hand, "strong" liquids (small fragility) exhibit sluggish kinetics and high viscosity in the supercooled regime, which delays the formation of crystal nuclei in the melts. Thus, the crystal growth is suppressed by the poor mobility of constituent atoms, leading to high thermal staibility and GFA of the supercooled liquid. The fragility is often associated with other characteristics of glasses, which is illustrated in Table 1.1. During supercooling, the crystallization speed is determined by the competition between the increasing driving force $(\Delta \mathrm{G})$ and the increasing resistance to structural rearrangements or molecular transportation (viscosity $\eta$ ). The competition between these two parameters determines whether alloys are good glass formers.

Table 1.1 Correlations between selected properties and the fragility of the supercooled liquids in glass forming systems [39].

\begin{tabular}{cccccc}
\hline \hline $\begin{array}{c}\text { Glass } \\
\text { characteristic }\end{array}$ & Viscosity & $\begin{array}{c}\text { Glass- } \\
\text { forming } \\
\text { ability }\end{array}$ & $\begin{array}{c}\text { Fracture } \\
\text { toughness }\end{array}$ & $\begin{array}{c}\text { Possion's } \\
\text { ratio }\end{array}$ & $\begin{array}{c}\text { Gibbs free } \\
\text { energy }\end{array}$ \\
\hline $\begin{array}{c}\text { Kinetically } \\
\text { strong } \\
\text { Kinetically } \\
\text { fragile }\end{array}$ & High & High & Low & Low & Small \\
\hline \hline
\end{tabular}




\subsubsection{Mechanical properties}

One of the enduring attractions of MGs is their promising mechanical properties, such as superior specific strength, high hardness, good ductility in bending, high wear and corrosion resistance, low friction coefficient etc. [40-42]. However, these superior properties are accompanied by their low tensile/compressive ductility at room temperature, owing to the lacking of dislocation-mediated crystallographic slip. The deformation mechanism of MGs is mostly attributed to shear banding, a particular deformation mode leading to their brittleness and catastrophic failure due to the free propagation of the shear bands [11]. In considering the mechanical performances of MGs, the key is to comprehensively understand shear bands, including the initiation, propagation, evolution, consequences and control [43].

During the deformation of MGs, strain can be easily accommodated by the changes in atomic neighborhood, due to the prevalence of metallic nature in MGs [44]. With crystal dislocations, the rearrangement of neighboring atoms could occur at low stresses or energies in crystals. However, the motion of neighborhood in MGs is a relatively highstress or high-energy process.

At the atomic level, the local rearrangements of atoms around free volume regions are believed to accommodate the shear strain in MGs under an applied stress. Such rearrangements are schematically depicted in Figure 1.5(a), which was originally proposed by Argon et al. [45]. The shear event in the figure has been named as a "flow defect" [46] or "shear transformation zone" (STZ) [47]. The STZ involves a group of atoms that experience a shear distortion from one low energy (equilibrium) state to a second such state, traversing an activated configuration with high energy. After a localized distortion is created, this shear strain continuously propagates, leading to the 
formation of so-called "shear bands". It is essential to stress that STZ is not a structural defect in MGs, but rather a transient state affected by local atomic rearrangements [2].
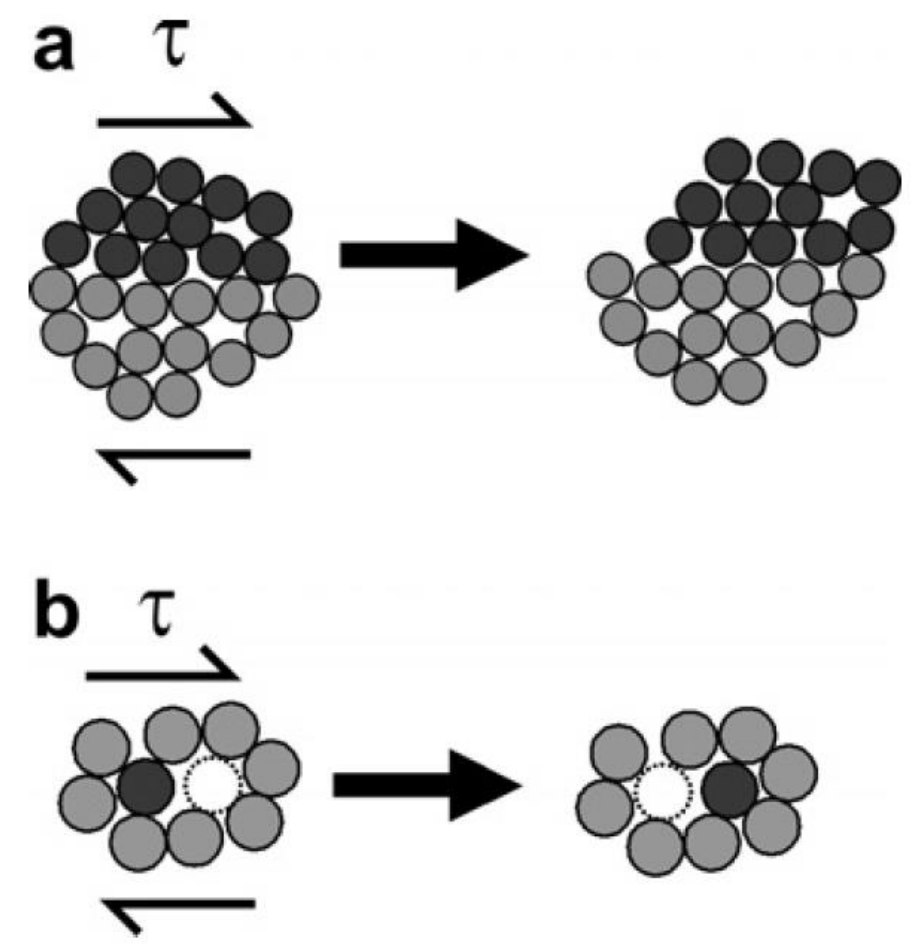

Figure 1.5 Schematics of the deformation mechanisms in amorphous alloys, including (a) a shear transformation zone [45], and (b) a local atomic rearrangement based on freevolume model [48].

An alternative explanation of the microscopic mechanisms of plastic flow in MGs is the "free volume" model proposed by Spaepen et al. [48, 49]. This theory views deformation as many diffusion-like atomic jumps into vacancies in MGs, as schematically illustrated in Figure 1.5(b). Obviously, these atomic jumps occur mostly near to regions with high free volume. In addition, the competition between free volume annihilation and creation has been proven to be a key factor determining the deformation features of glasses. If the applied stress exceeds a critical value, the annihilation rate will not keep up with the free volume creation, leading to strain softening and shear 
localization in MGs. These two models qualitatively outline the atomic level mechanisms underlying the deformation of MGs.

Due to the lack of long-range translational geometry, MGs are typically considered to be elastically isotropic. Isotropic materials possess two independent elastic constants, typically regarded as Possion's ratio $(v)$ and Young's modulus $(E)$. The bulk modulus (B) and shear modulus $(\mu)$ are more useful for understanding the elastic properties of MGs, since they respectively represent the responses of materials to hydrostatic and shear stresses. These two parameters involve bond stretching and distortion, respectively. The bulk modulus of glassy metals is typically $6 \%$ lower than that of corresponding crystalline compounds [50, 51]. For crystalline alloys, the curvature of interatomic potential and the equilibrium interatomic distance together determines the bulk modulus [52]. The density of MGs has been proven to be slightly smaller than that of crystals, and thus the average interatomic spacing is slightly higher in glasses. Such difference can explain the lower bulk modulus of MGs. In contrast, the shear modulus in amorphous alloys is about $30 \%$ lower than that of crystals [53-55]. The difference in atomic spacing is inadequate to explain such a large difference in shear modulus. Under a shear strain, every atom in the crystals undergo an affine displacement defined by the macroscopic strain, while the local atomic displacement in glasses deviates from the affine projection due to the amorphous structure $[2,12]$. These local rearrangements of atoms reduce the shear modulus of MGs by about $30 \%$, as proved by many simulations $[56,57]$. 


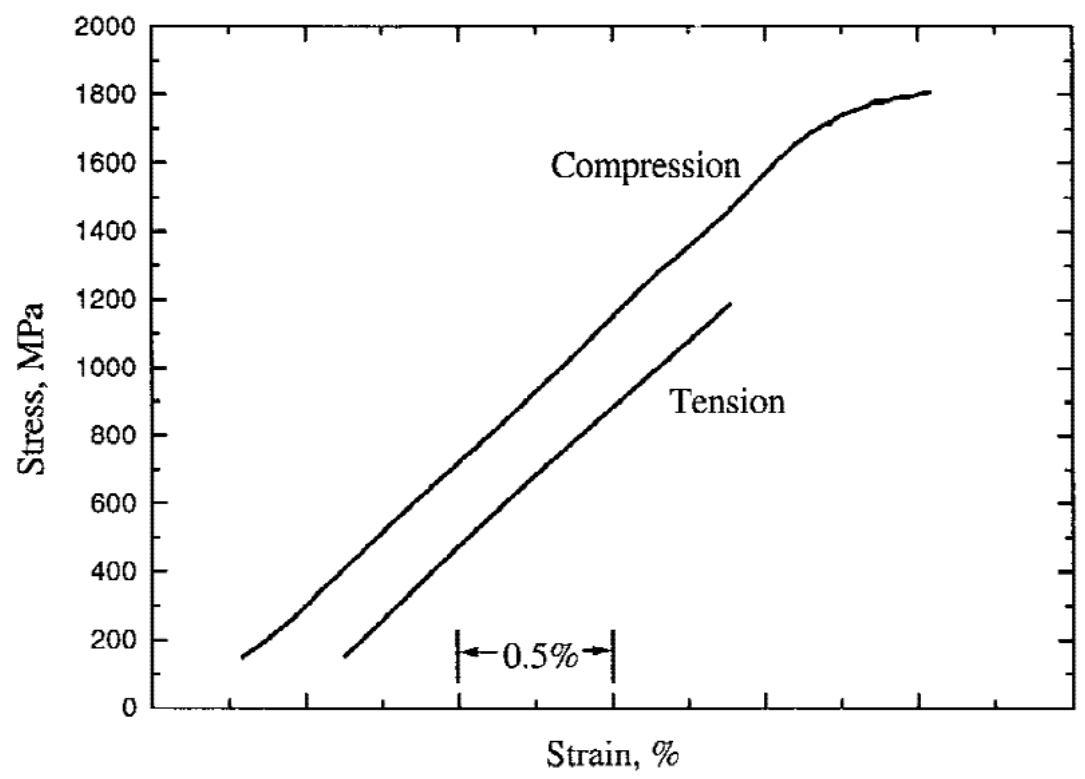

Figure 1.6 Compressive and tensile stress-strain curves of $\mathrm{Zr}_{57} \mathrm{Nb}_{5} \mathrm{Cu}_{15.4} \mathrm{Ni}_{12.6} \mathrm{Al}_{10}$ amorphous alloy [58].

A unique characteristics of MGs is their ultrahigh strength, as their elastic limit can reach as high as 1 to $2 \%$ [59]. In addition, flow serrations have been found in the loaddisplacement response of MGs, which represents a relaxation event related with the shear band formation [60]. The serration behavior is also associated with the temperature [61]. As the strain raises to a critical value, a shear band is activated and quickly propagates in MG samples, leading to the catastrophic failure [62]. Another characteristic of MGs is their different responses to tension and compression, as described in Figure 1.6 [58]. No plasticity under tensile loading is observed in the stress-strain curves, whereas small plastic strain under compression is found in the specimen. Under compression, large shear strains can be accommodated by multiple shear bands, giving MGs limited ductility. Under tension, however, glasses fail by the shear rupture in a primary shear band with little plastic strain. The compressive plasticity in MGs is attributed to the slipping, branching and intersecting of multiple shear bands. 
The fracture under compression mainly occurs at an angle of 42 to $43^{\circ}$ from the loading axis, whereas the tensile fracture angle is about $56^{\circ}$, as seen in Figure 1.7 [63].

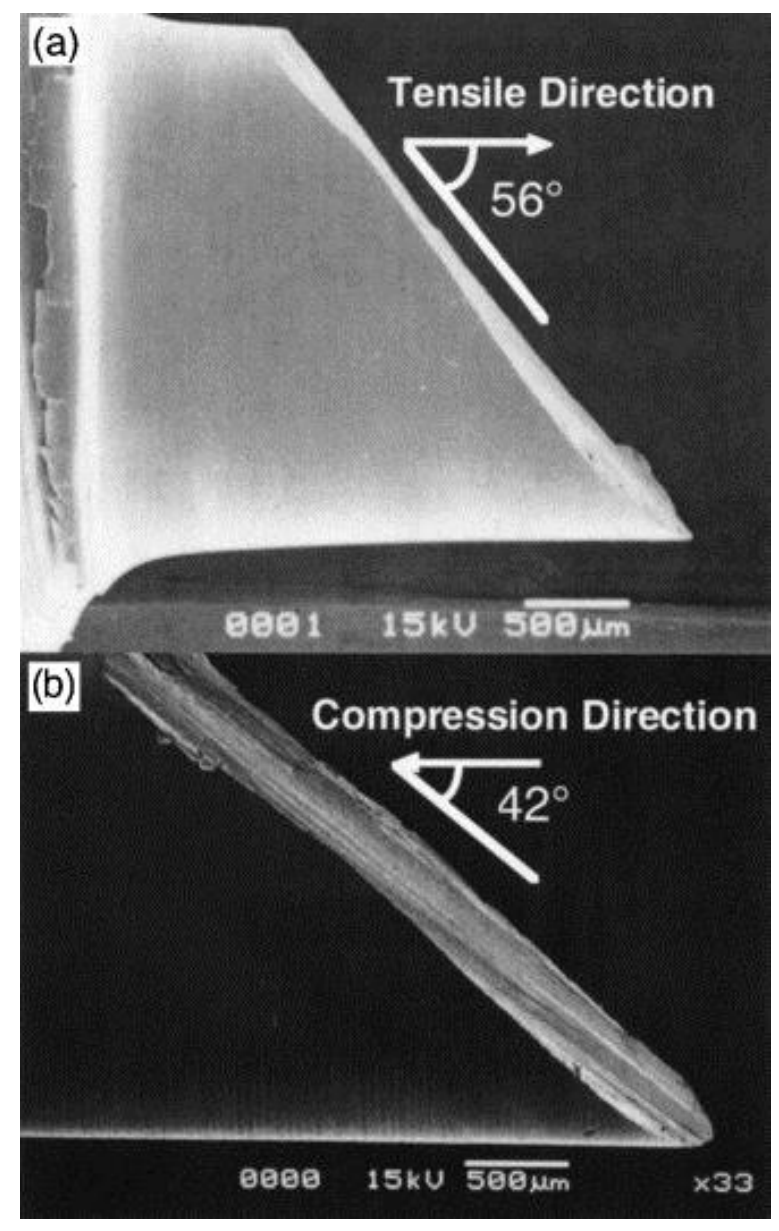

Figure 1.7 The fracture behaviors of $\mathrm{Pd}_{40} \mathrm{Ni}_{40} \mathrm{P}_{20}$ glasses under (a) tension and (b) compression, illustrating the difference in fracture angles [63].

\subsection{Microscopic structure of metallic glasses}

Macroscopically, MGs have amorphous structure with no long-range translational order. At the atomic level, topological and chemical short-to-medium range order are believed to be prominent in glassy alloys, owing to their high packing efficiency and different chemical affinity between constituents [12]. The fundamental knowledge on the MG structure has been a long-standing subject of interest for understanding the thermodynamic, kinetic and mechanical properties. 


\subsubsection{Short-range order}

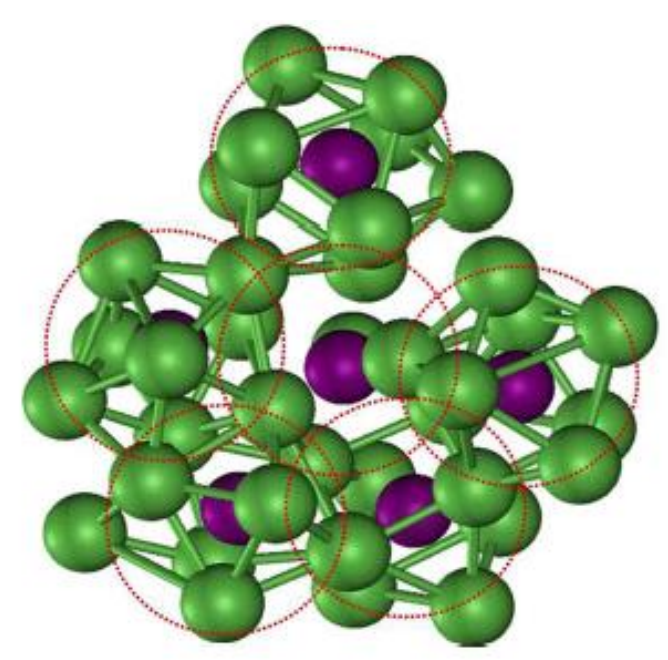

Figure 1.8 Solute-centered clusters discovered in the simulated $\mathrm{Ni}_{81} \mathrm{P}_{19}$ amorphous alloy [64]. B and Ni atoms are colored purple and green, respectively. The MRO is formed by the interconnection of those quasiequivalent clusters.

Many experimental and simulation studies support the significance of solute-centered atomic clusters in the structure and stability of amorphous alloys. These atomic clusters exhibiting similar structural features are commonly referred to as the short-range order (SRO) in MGs. In binary or multicomponent MGs, constituent elements are sometimes classified into solute and solvent atoms according to their fractions. The major and minor elements are respectively considered as solvent and solute atoms. In particular, clusters formed by a center solute atom and several neighboring solvent atoms have been proven to be the representative structural elements in many solute-lean systems, such as $\mathrm{Ni}_{81} \mathrm{~B}_{19}, \mathrm{Ni}_{80} \mathrm{P}_{20}$ and $\mathrm{Zr}_{84} \mathrm{Pt}_{16}$ [64]. In these systems, solute-solute connections diminish due to the low fraction of solute element and the varying chemical affinity between constituent elements [65]. Such phenomenon is typically named as "solute-solute avoidance", which is an intrinsic characteristic of many MGs [12]. If the proportion of solute atoms exceeds a critical value, the neighboring atoms would become a mixture of 
solute and solvent atoms. A typical example of solute-centered clusters at atomic level is depicted in Figure 1.8, which shows the $\mathrm{SRO}$ in $\mathrm{Ni}_{81} \mathrm{~B}_{19}$ glass (red circles). As seen in the figure, solute B atoms are completely surrounded by solvent $\mathrm{Ni}$ atoms, making up the solute-centered clusters in the system.

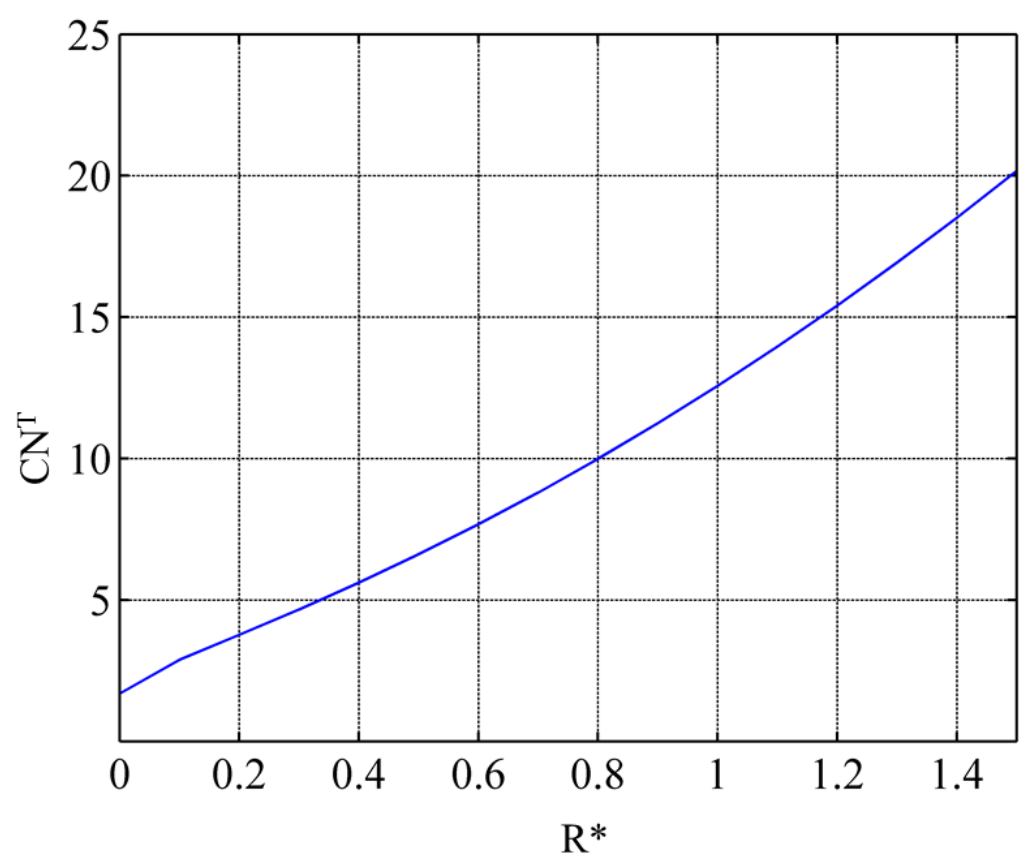

Figure 1.9 Theoretical coordination number of an element $i$ in liquid of element $j$ as a function of the radius ratio $\mathrm{R}^{*}=r_{i} / r_{j}[66]$.

Owing to the high packing efficiency of MGs, the average coordination number $(\mathrm{CN})$ in MGs is controlled by the effective radius ratio between solute atoms and their neighbors, $R^{*}[66,67]$. The $\mathrm{CN}$ of a given atom describes the number of atoms that are in its first coordination shell. Unlike crystalline solids, the determination of nearest neighbors in amorphous system is somewhat arbitrary. Several different ways are typically used to define the nearest neighbors in MGs, such as distance cutoff and Voronoi tessellation techniques [12]. Take distance cutoff method for example, atoms contributing to the first peak of the corresponding radial distribution function (RDF) are considered as the 
nearest neighbors [68]. In amorphous alloys, the typical relationship between the predicted $\mathrm{CN}$ and $R^{*}$ is described in Figure 1.9. Computer simulations were employed to study how $R^{*}$ affects the local packing scheme in different MGs [64]. As seen in Figure 1.10, the preferential $\mathrm{CN}$ of the solute atom in $\mathrm{Zr}_{84} \mathrm{Pt}_{16}$ and $\mathrm{Ni}_{81} \mathrm{~B}_{19} \mathrm{MG}$ is 12 and 9 respectively. Such finding is directly attributed to the different $R^{*}$ in these two alloys, as shown in Figure 1.9. The $R^{*}$ of $\mathrm{Zr}-\mathrm{Pt}$ and $\mathrm{Ni}-\mathrm{B}$ systems are 0.9 (corresponds to a theoretical $\mathrm{CN}$ of 12) and 0.69 (theoretical $\mathrm{CN}$ of 9) respectively, and hence the dominant $\mathrm{CN}$ becomes different in these systems.

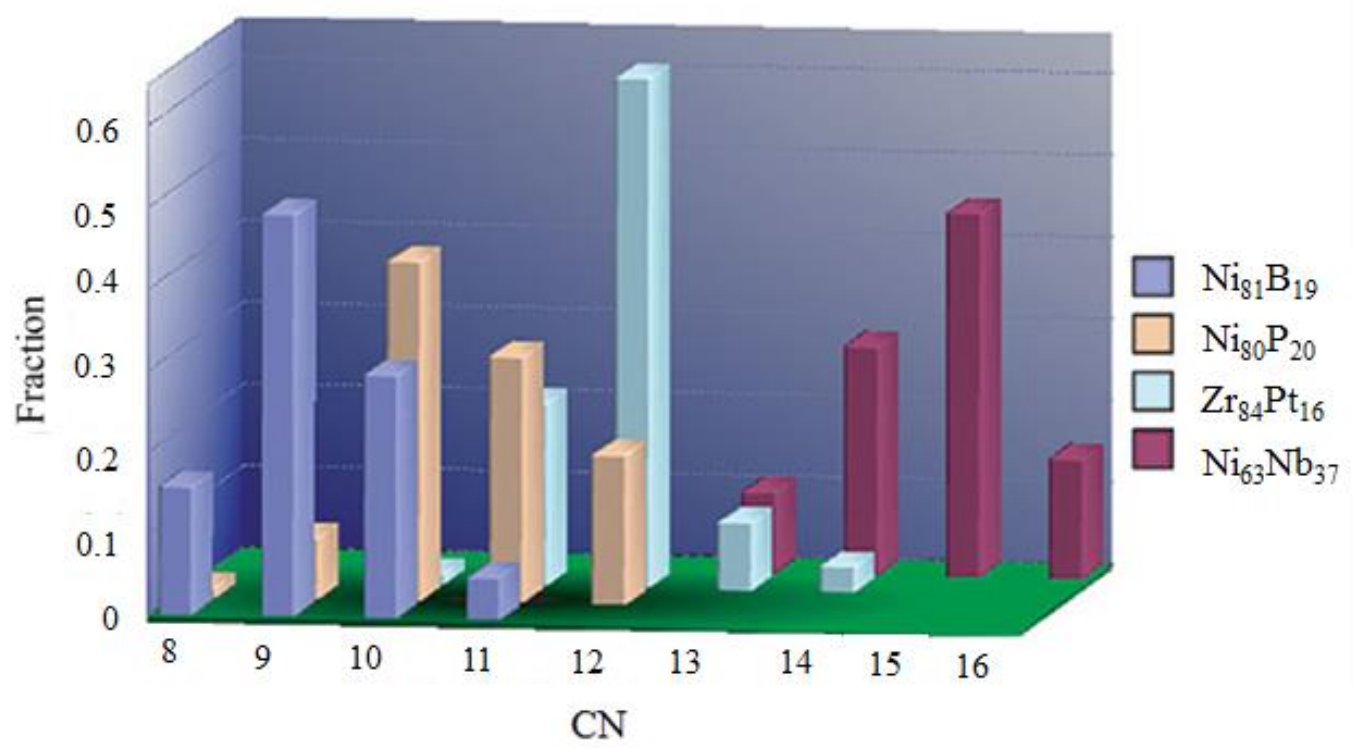

Figure $1.10 \mathrm{CN}$ distributions of the solute atoms in various MGs, based on ab initio calculations [64]. The average $\mathrm{CN}$ varies with the effective atomic radius ratio $R^{*}$. 


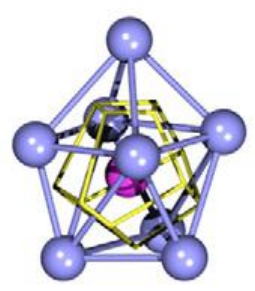

$<0,4,4,0>$

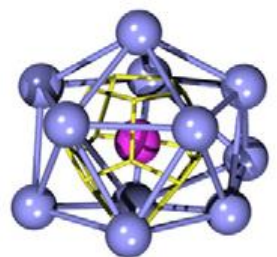

$<0,2,8,1>$

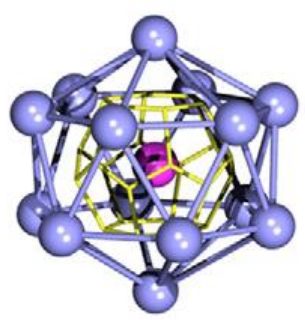

$<0,0,12,2>$

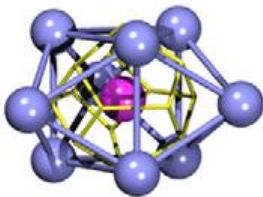

$<0,3,6,0>$

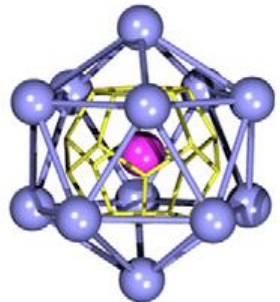

$<0,0,12,0>$

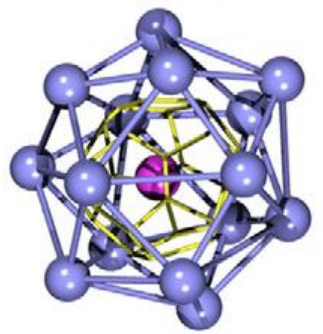

$<0,0,12,3>$

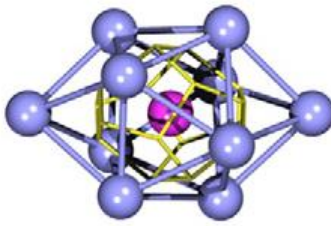

$<0,2,8,0>$

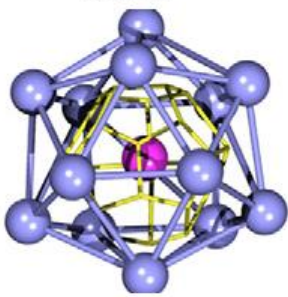

$<0,1,10,2>$

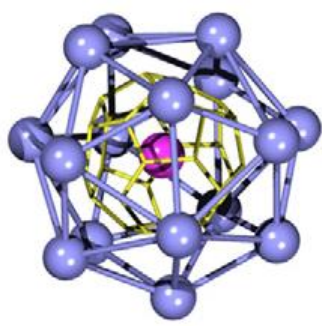

$<0,0,12,4>$

Figure 1.11 Several Voronoi polyehedra with different CNs [12]. Clusters are formed by the centeral and neighboring atoms, which are colored cyan and blue, respectively. The corresponding Voronoi polyhedra are depicted by the yellow lines.

In many simulation studies, researches have used the so-called "Voronoi index" to classify the solute-centered clusters $[69,70]$. The Voronoi tessellation method divides the 3D space into many isolated polyhedra (or cell) centered by each atom in glasses. Such technique can be employed to definitely define the $\mathrm{CN}$ in amorphous system: those atoms sharing the same cell surface are referred to as nearest neighbors. Additionally, the Vonoroi index, $\left\langle n_{3}, n_{4}, n_{5}, n_{6}\right\rangle$, can be used to describe the local arrangements and geometry of the nearest neighbors around the center atom, as illustrated in Figure 1.11. Specifically, $n_{3}$ demonstrates to the number of triangles on the Voronoi cell, $n_{4}$ the number of quadrangles, $n_{5}$ the number of pentagons, etc. For example, $\langle 0,0,12,0\rangle$ corresponds to a dodecahedron with twelve pentagonal faces, which is depicted in the 
figure. A typical example of Voronoi analysis is the simulation studies of $\mathrm{Cu}-\mathrm{Zr}$ MGs, which were extensively investigated due to their excellent GFA with a wide composition range $[71,72]$. Based on the Voronoi indices, the MD and reverse Monte Carlo (RMC) simulation results suggest the dominance of five-edged faces in $\mathrm{Cu}-\mathrm{Zr}$ MGs. Among the numerous clusters around $\mathrm{Cu}$ atoms, the $\mathrm{Cu}$-centered full icosahedra (FI) with Voronoi index $\langle 0,0,12,0\rangle$ have attracted particular interest, since their proportion changes dramatically in response to varying $\mathrm{Cu}$ content [73]. Moreover, their fraction is dramatically sensitive to the cooling rate [71]. The essential role of $\mathrm{Cu}$-centered FI in determining MG attributes will be further outlined in Section 1.4.

\subsubsection{Medium-range order}

The orderliness of MG structure can still be found at a larger scale, which is generally referred to as the MRO. Despite solute-centered clusters in MGs possess different topologies and CNs, they can be considered quasi-equivalent, due to their similar sizes and CNs [64]. Computer simulations show that quasi-equivalent clusters are likely to be stacked with icosahedral order, giving rise to the MRO. Figure 1.8 also shows an icosahedral supercluster in $\mathrm{Ni}_{81} \mathrm{~B}_{19}$ MG. The B-centered clusters are percolated with one another by sharing the solvent atoms, forming the MRO. Additionally, the icosahedraltype MRO is not found to be affected by the SRO types. For instance, $\mathrm{Zr}_{84} \mathrm{Pt}_{16}$ (the dominant $\mathrm{SRO}$ is $\langle 0,0,12,0\rangle$ ) and $\mathrm{Ni}_{81} \mathrm{~B}_{19}$ (the dominant $\mathrm{SRO}$ is $\langle 0,3,6,0\rangle$ ) MGs exhibit similar types of MRO. Very recently, the distribution of $\langle 0,0,12,0\rangle$ clusters in $\mathrm{Cu}-\mathrm{Zr} \mathrm{MGs}$ are found to be heterogeneous [71]. These clusters preferentially connect with one another, forming chains and networks, which may provide another example of the medium-range structure in MGs. 


\subsection{Structure-property relationship}

Due to the amorphous nature of MGs, their structure-property correlation remains a long-standing difficulty to scientists. Nevertheless, researchers have made considerable efforts devoted to this topic, and encouraging progress has been achieved over the years. Numerous studies suggest that the properties of MGs are predominantly dependent on their internal structures, such as the changes of alloy composition, or variations of processing histories. The microscopic structure associated with the glass properties has been derived from atomistic simulations. This section provides a brief summary of recent progress in this important area.

\subsubsection{Structural-thermodynamic relationship}

The microstructure is a key factor controlling the diffusion and structural relaxation of MGs and supercooled liquids, and therefore affects their thermodynamics and kinetics behaviors. The relaxation dynamics of liquids slows down under supercooling, and hence the atomic motion becomes different and heterogeneously distributed in space. Some atoms are faster and can transport in the supercooled liquids, whereas some are locally encaged and immobile. The dynamical heterogeneity is intimately connected with the microstructure distribution, and has been extensively studied by atomistic simulations [74-76]. Doye and Meyer [77] investigated the energetically favored clusters in a binary glass. By adjusting the relative size of atoms, it is found that different polytetrahedral clusters can be favored. In addition, the energetic stability is associated with both topological and chemical order. The representative topological order is denoted by extracting the Voronoi index, while the chemical order can be characterized by chemical short range order (CSRO) parameters. In binary and multicomponent glasses, the most favored SRO is typically characterized by clusters with high symmetry and chemical order [12]. 
A recent simulation study stressed the significance of icosahedral ordering in $\mathrm{Cu}-\mathrm{Zr}$ MGs. As temperature decreases, one can observe that the proportion of $\mathrm{Cu}$-centered FI $(<0,0,12,0\rangle)$ dramatically increases towards glass transition [73]. In addition, the $\mathrm{Cu}-$ centered FI is directly associated with the local atomic mobility, as shown in Figure 1.12 [73]. The majority of immobile atoms are found to be associated with the $\langle 0,0,12,0\rangle$ clusters. Similar results regarding the dynamics-structure correlations have also been carried out by many researchers $[78,79]$. Accordingly, the icosahedral order with high degree of fivefold symmetry may explain the structural aspects of the thermodynamics and kinetics of MGs.

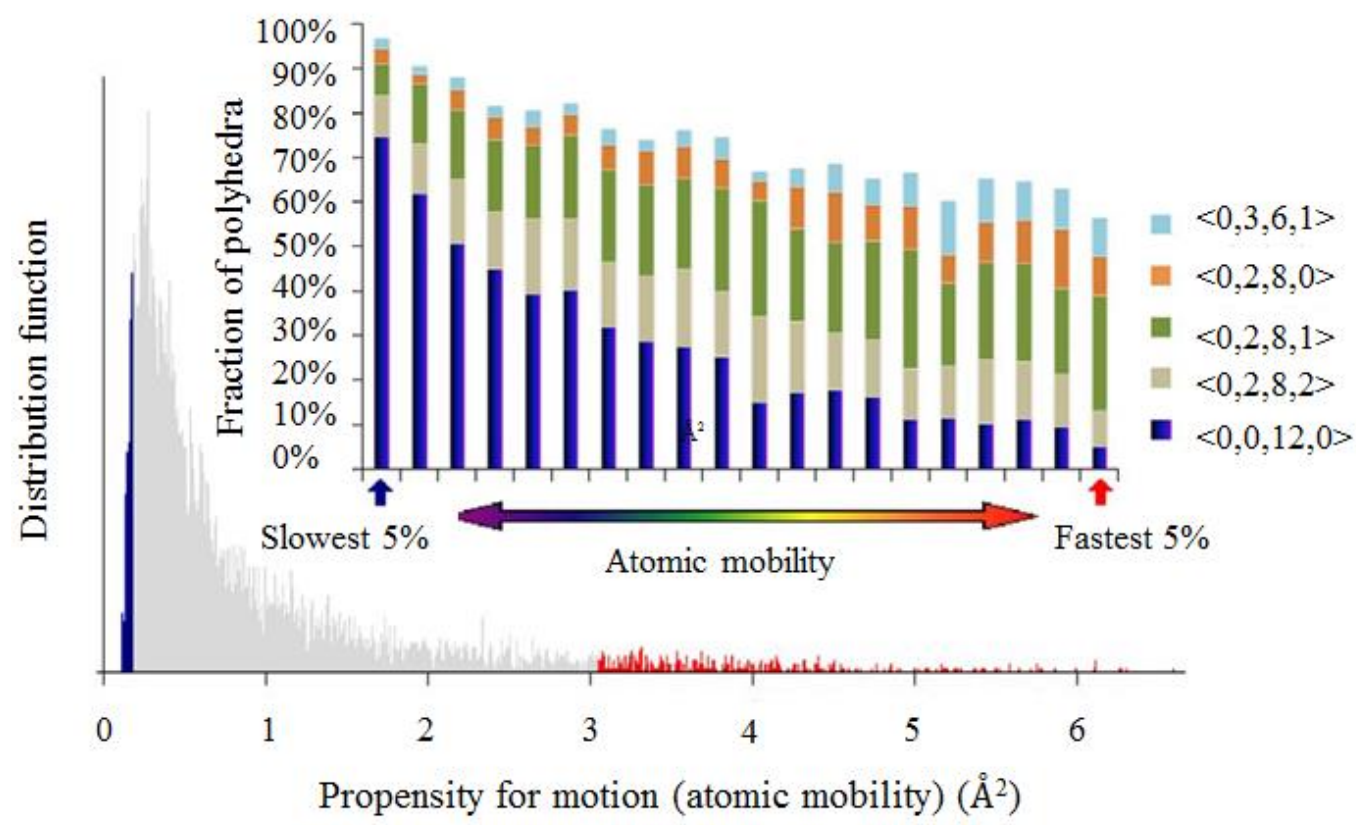

Figure 1.12 Distributions of atomic mobility (propensity for motion) of $\mathrm{Cu}$ atoms in $\mathrm{Cu}_{64} \mathrm{Zr}_{36}$ supercooled liquid (at $800 \mathrm{~K}$ ) [73]. Right and left sides denote the $5 \% \mathrm{Cu}$ atoms having the fastest and slowest mobility, respectively. In the inset, $\mathrm{Cu}$-centered polyhedra are sorted according to the atomic mobility (low to high) and then separated into 20 groups, each involving $5 \%$ of the total $\mathrm{Cu}$ atoms. 


\subsubsection{Structural-mechanical relationship}

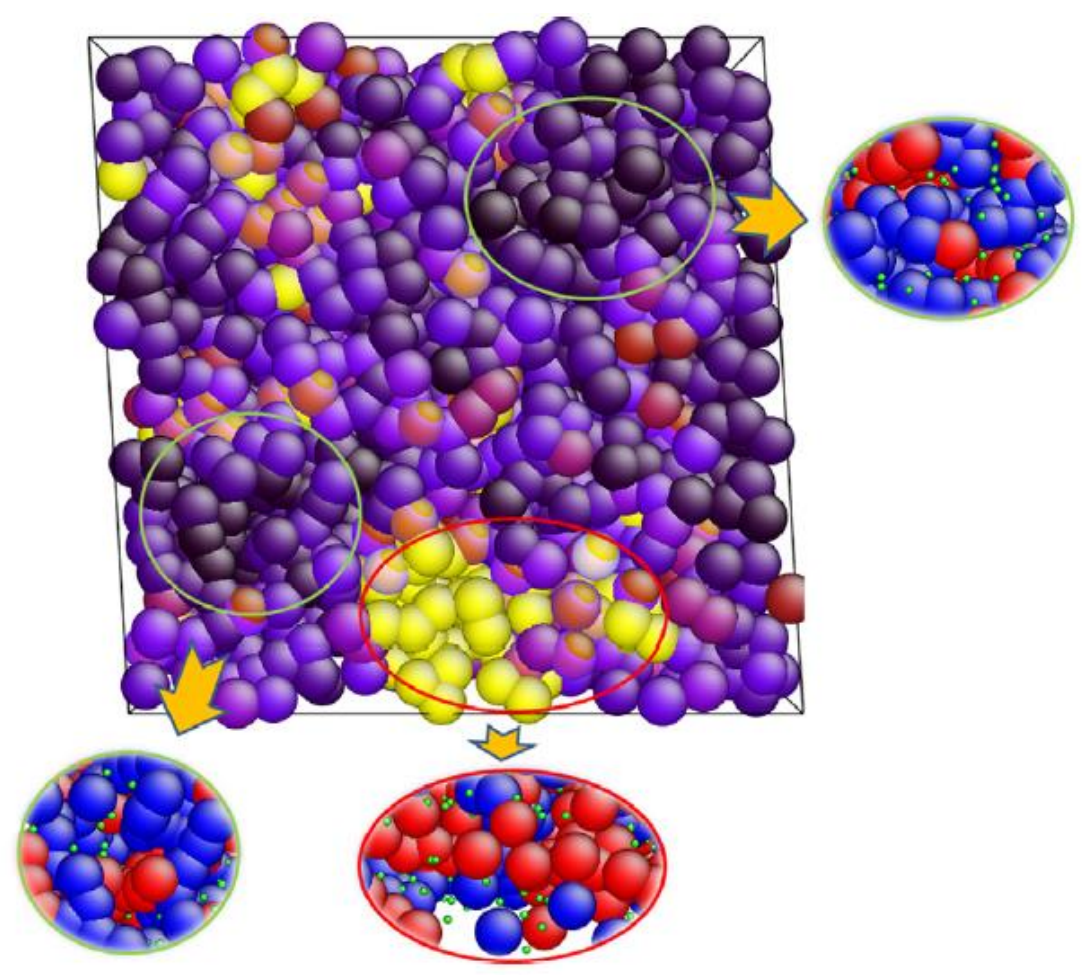

Figure 1.13 Relationship between icosahedral clusters and local inelastic deformations (STZ) [80]. Such figure displays the cross-section view of a sheared $\mathrm{Cu}_{64} \mathrm{Zr}_{36}$ sample. The configuration only depicts $\mathrm{Cu}$ atoms for clarity, indicating the $\mathrm{Cu}$-centered polyhedra. The coloring schemes are assigned according to the different values of von Mises shear strains. The elastically and plastically deformed and regions are colored dark purple and yellow respectively. The circles represent two elastic entities and one STZ, and their local structures are illustrated in the insets. The $\mathrm{Cu}$ atoms in the elastic entities are mostly in $\langle 0,0,12,0\rangle$ FI (such $\mathrm{Cu}$-centered polyhedra are mapped blue), while the $\mathrm{Cu}$ atoms in the STZ are mostly embedded in unfavorable (liquid-like) clusters (mapped red). The $\mathrm{Cu}$ atoms of other regular clusters are displayed by small green dots.

The unique mechanical properties of MGs are characterized by the high strength and limited plasticity, which are intimately connected with the atomic-level structure. Due to the intrinsic structural fluctuations in MGs, the microstructure changes from site to site. 
Similar to the structure-kinetics correlations, the structural heterogeneity gives rise to the mechanical heterogeneity in MGs. The mechanical heterogeneity is typically measured by local stiffness in glasses, which can be characterized by computer simulations and experiments. Specifically, regions with low stiffness are unstable (or liquid-like) and preferential for localized shear transformation, which may lead to the shear band formation in realistic MGs $[81,82]$. With external loadings, STZs with low stiffness are locally activated to undergo inelastic deformations [2]. The subsequent percolation of STZs results in the shear band formation, accompany with the catastrophic failure of MGs. The correlation between microstructure and local stiffness has been revealed by atomistic simulations [83, 84]. The deformation behavior of CuZr-based MGs is found to be affected by the icosahedral order, as shown in Figure 1.13 [80]. Various simulation studies suggested that icosahedral clusters correspond to regions with higher shear resistance and stiffness, while the disordered atoms exhibit low stiffness and are fertile sites for local shear deformation $[85,86]$.

If a MG sample is deformed elastically, the local plastic deformation of the defects (liquid-like sites) is confined. These STZs are hence isolated and embedded in the specimen, and can be recovered upon unloading [2]. Then if the loading stress is so large that many STZs are activated to percolate with one another, the unstable region will reach a critical scale and give rise to irreversible macroscopic strain. Once irreversible shear occurs, the shear front will propagate quickly in the matrix, leading to instability and eventually catastrophic failure. A recent experiment by Schall et al. [87] confirmed the observation of STZ percolation in colloidal glasses. In addition, many simulation studies have been conducted to unravel the mechanisms and process of shear band formation in MGs. For instance, Cao et al. [88] successfully examined the yielding of MGs by using MD simulations. The results suggested the STZ activation around the free 
surface, the STZ percolation, the collapse of icosahedral backbone, and their correlations. Once the shear band propagates, icosahedra in the shear band are found to be broken and transformed into unstable and fragmented clusters.

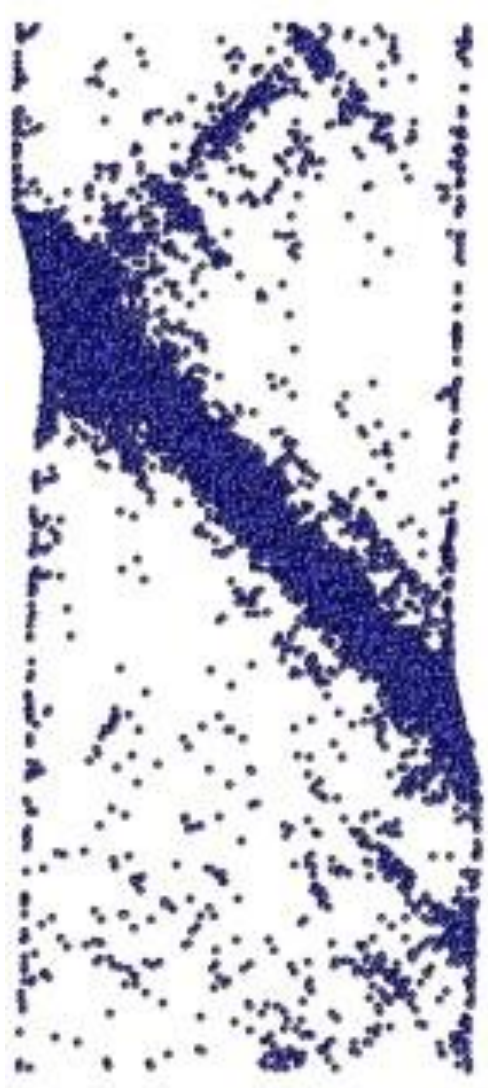

(a)

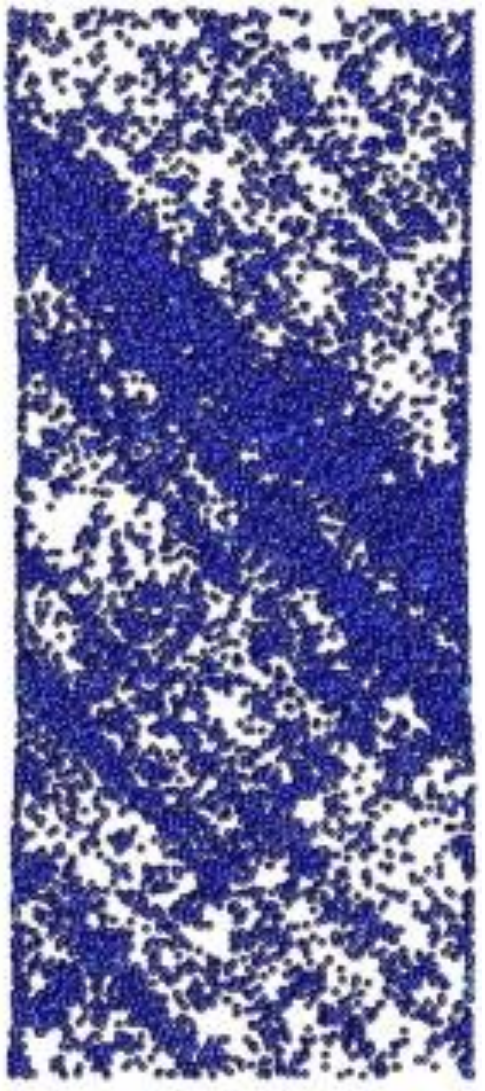

(b)

Figure 1.14 Comparison of the deformation behaviors in terms of shear localization in (a) $\mathrm{Cu}_{64} \mathrm{Zr}_{36}$ and (b) $\mathrm{Cu}_{40} \mathrm{Zr}_{60}$ glasses [89]. Only atoms with atomic shear strain higher than 0.3 are shown for clarity.

The plastic deformation of MGs is originally triggered by the STZ activation. The STZs are energetically unfavored and exhibit low stiffness and stability. Thus these STZs are liquid-like and their effect on plasticity in MGs is of fundamental importance. It was reported that MGs with more liquid-like sites preferentially undergo homogeneous flow, while strain localization is favored in glasses with more solid-like structure. It is 
therefore proposed that the deformation mode (homogeneous flow or localized shear band) is mainly determined by the process and percolation of STZs. Therefore, the intrinsic plasticity of $\mathrm{MGs}$ is directly related to the relative proportion, spatial distribution and property of STZs, as proven by recent simulations [88, 89]. For example, the different degrees of strain localization in $\mathrm{Cu}_{64} \mathrm{Zr}_{36}$ and $\mathrm{Cu}_{40} \mathrm{Zr}_{60}$ MGs are proposed to be related with the different population and connectivity of those STZ fertile sites [89]. Compared to the $\mathrm{Cu}_{64} \mathrm{Zr}_{36}$ sample, the $\mathrm{Cu}_{40} \mathrm{Zr}_{60}$ alloy exhibits a more homogenous plastic deformation and better intrinsic plasticity, as shown in Figure 1.14. As mentioned before, the icosahedral clusters in $\mathrm{CuZr}$ MGs are stable and highly resistant to local plastic deformation, while the disordered clusters exhibit low stiffness and become fertile sites for STZs. Due to the high population of icosahedral clusters founded in $\mathrm{Cu}_{64} \mathrm{Zr}_{36}$, the plastic strain in this sample tends to concentrate in the initiated shear band rather than diffusing into nearby space. Similarly, the quenching rate dependences of mechanical features in MGs are attributed to the degrees of structural ordering and defects. For instance, the proportion of icosahedra in $\mathrm{Cu}-\mathrm{Zr}$ MGs increases significantly with decreasing quenching rates [71]. Hence, samples with more icosahedral order are found to exhibit high strength, high shear modulus, and low intrinsic plasticity.

\subsection{Metallic glass matrix composites}

The main drawback of monolithic BMGs is their intrinsic room-temperature brittleness, which is attributed to the rapid propagation of activated shear band. Due to the lack of crystal defects, plastic deformation of MGs is typically concentrated in shear bands with a thickness of only $10 \mathrm{~nm}[2,90]$. To eliminate the brittleness of monolithic BMGs, metallic glass matrix composites (MGMCs) have been synthesized during the past decades [3]. The presence of secondary crystalline phases can directly prompt the multiplication of shear bands and suppress the shear band propagation. In general, two 
kinds of composites have been extensively studied: in-situ composites, where crystalline phases are precipitated during solidification, and ex-situ composites, where crystalline particles or fibers are added into a melt before casting. The characteristics and mechanical properties of in-situ and ex-situ MGMCs will be the discussed in this section.

MGMCs could be classified into two groups: extrinsically and intrinsically formed composites. The secondary phases of these composites could exhibit different microstructural features and length scales. Typically, the ex-situ composites are synthesized by directly adding crystalline reinforcements during the processing of glassforming melts. The reinforcements consist of either particles or fibers [91, 92]. For example, W, Ta, Nb, Mo, and $\mathrm{SiC}$ crystalline particles have been successfully introduced to the Vit-106 melt to synthesize particulate-reinforced composites $[58,91$, 93]. The achievable microstructures of in-situ composites possess distinct shape and morphology, such as nanoscale quasicrystals, nanocrystalline particles, dendritic precipitates, or two amorphous phases through the phase separation [3]. Figure 1.15 shows the microstructure of an in-situ MGMC containing $\beta$-phase. The X-ray diffraction (XRD) peaks shown in the inset is attributed to the body-centered cubic (bcc) phase. 


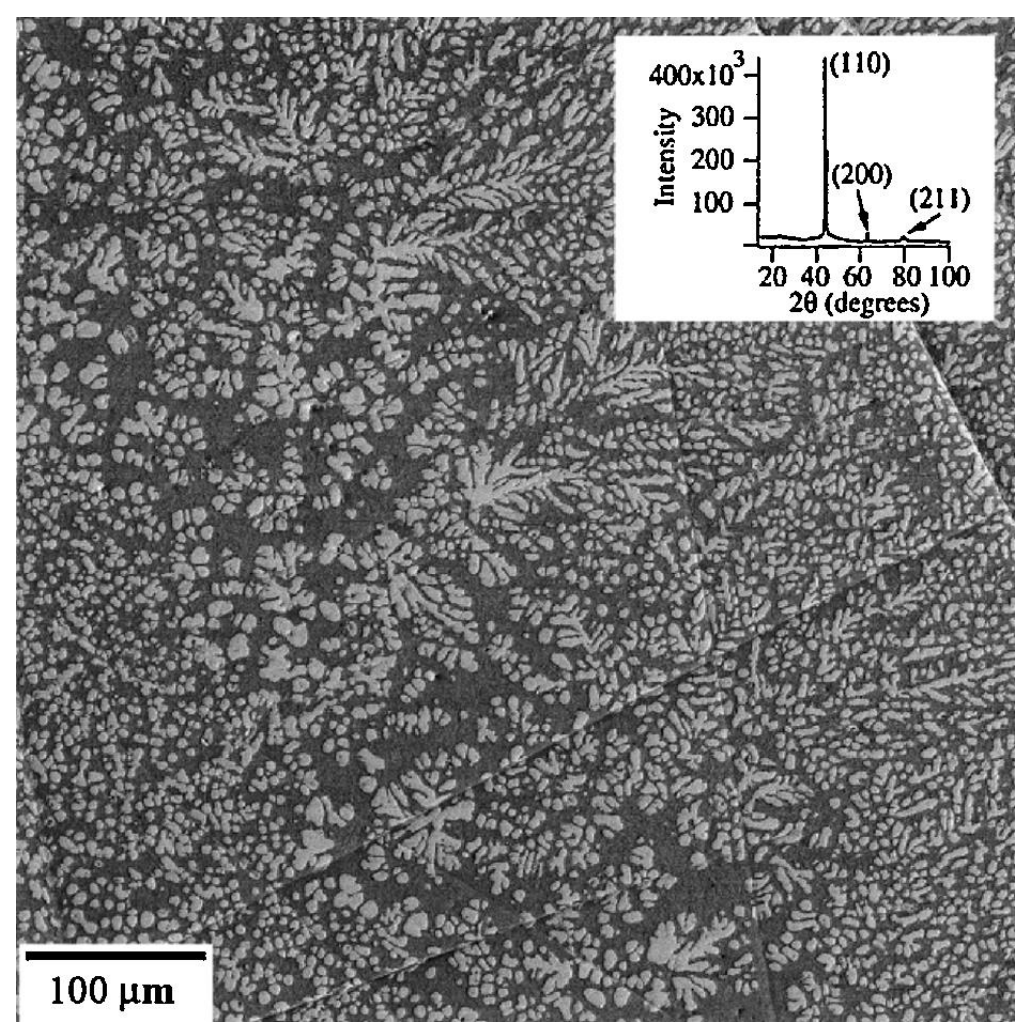

Figure 1.15 Scanning electron microscopy (SEM) image of the microstructure of the $\left(\mathrm{Zr}_{75} \mathrm{Ti}_{18.34} \mathrm{Nb}_{6.66}\right)_{75} \mathrm{X}_{25}$ in-situ composites [94].

The key factors governing the strength and plasticity of MGMCs include the reinforcement properties, the properties of the interface between glass matrix and secondary phases, the volume fraction, morphology and size of the secondary phases. Usually, the fracture strength of ex-situ composites could be improved with increasing amount of reinforcement $[95,96]$. However, the reinforcing effect of the secondary phases tends to diminish as the volume fraction exceeds a critical value [97]. Despite the high fracture strength achieved in most ex-situ composites, their plastic strain is yet limited to 1-5\%. An exception is the Zr-based BMGs mixed with $\mathrm{W}$ filaments, for which a strain limit up to $18.5 \%$ has been achieved [98]. The extraordinary plasticity of such material is primarily attributed to the ductile property of the $\mathrm{W}$ particles. The mechanical performance of in-situ composites mainly depends on several parameters: the morphology, the size and the volume fraction of secondary phases. For instance, 
partially crystallized $\mathrm{Zr}_{57} \mathrm{Cu}_{20} \mathrm{Al}_{10} \mathrm{Ni}_{8} \mathrm{Ti}_{5}$ alloys were synthesized and investigated by annealing the monolithic glass [99]. The nanoprecipitates of these composites are homogeneously embedded in the matrix, with a characteristic length of 2 to $10 \mathrm{~nm}$. The results suggest that the strength of the material is strongly improved with the introduction of crystallites, while the plastic limit is slightly affected. In another case, composite microstructure was achieved by adding 1 at.\% Si to a $\mathrm{Cu}-\mathrm{Ti}-\mathrm{Zr}-\mathrm{Ti}$ glass [100]. The composite consists of nanoparticles about 8 to $12 \mathrm{~nm}$ in size, which are homogeneously distributed in the matrix. On the other hand, star-shaped precipitates with a size of 3 to $5 \mu \mathrm{m}$ were found by adding 1 at.\% Sn into the same composition [100]. The Cu-Ti-Zr-Ti BMG and $\mathrm{Cu}-\mathrm{Ti}-\mathrm{Zr}-\mathrm{Ti}-\mathrm{Si}$ composite display little ductility, whereas the $\mathrm{Cu}-\mathrm{Ti}-\mathrm{Zr}-\mathrm{Ti}-\mathrm{Sn}$ composite shows a plastic strain limit up to $2.2 \%$.

Compared to MGMCs with nano and micron-scale crystalline particles, composites containing dendritic phases typically exhibit higher strength and better ductility [101]. Such composites exhibit improved plastic behavior in tension, compression and bending. For $\mathrm{Cu}$-based BMGs, a small volume of dendritic precipitates is adequate for promoting the plasticity [101]. For La-based and Zr-based MGMCs, plasticity enhancement was detected when the volume fraction of crystalline inclusions reaches a critical value [102, 103]. An interesting finding is that the activated shear bands in these samples tend to intersect with dendritic arms before propagation, which may attribute to the inhibition of shear band propagation. Unfortunately, only a few systems have been proven to gain MGMCs with dendritic phases, and the possible composition range is still limited. Moreover, the dendrites are strongly dependent on the cooling histories, leading to the microstructural variations during casting. Furthermore, another drawback of dendritic composites is their slightly reduced yield strength compared to monolithic BMGs. 


\subsection{Applications}

MGs possess certain remarkable attributes, and hence are attractive materials for commercial applications. MGs are extensively used for making sport equipment, due to their advantages like low density, low damping and high specific strength. With such merits, MGs are exploited in high-end sporting equipment, such as golf plates, tennis racquets and bicycle frames. In addition, BMGs are promising in the electronic market, due to the applicability of fabrication into fine components. Another use of commercial applications involves the medical equipment such as surgical instruments, bone plates and dental implants. For example, Ti-based BMGs are particularly suitable for orthopedic applications due to the unique properties, including biocompatibility, excellent wear resistance, high specific strength and good thermal formability. Moreover, the outstanding thermal formability of MGs provides an opportunity for the exploitation in micro-electro-mechanical systems (MEMS) [104]. The ultrahigh strength and lack of crystal defects of BMGs imply their superior performances in MEMS compared to crystalline alloys. Traditional fabrication methods for MEMS devices include lithography and chemical or ion etching, which are either expensive or lack fabrication accuracy. With good thermal formability, the required nano-patterns of MEMS devices can be easily acquired through plastic processing techniques. One case of commercial applications is the micro gears fabricated by using die-forging approaches [105].

\subsection{Motivation of this thesis}

Although MGs exhibit superior properties and hence are promising materials for industrial applications, there are still drawbacks. Therefore, it is essential to explore the intrinsic structure in relation with their physical and mechanical characteristics, especially in terms of chemical and topological aspects. Until now, numerous works over the past decades have addressed a number of key issues, such as the prevalence of 
icosahedral ordering in some binary and multicomponent glasses. However, many unsolved puzzles remain in the field of microstructure and structure-property correlations in amorphous alloys.

The free volume concept and icosahedral ordering have been extensively used for understanding the behaviors of MGs. In some cases, the application of free volume or icosahedral ordering may still be questionable. As discussed by Egami et al. [66], the main limitation of free volume theory is ascribed to the relative compressibility of atoms. The dominant Voronoi polyhedron is also found to vary with alloy systems and compositions. Hence the explanation based on icosahedral ordering becomes inapplicable in some glass systems. For instance, $\mathrm{Cu}$-centered full icosahedra are reported to exhibit dynamic instability in $\mathrm{Cu}_{20} \mathrm{Zr}_{80}$ glass [106]. In this regard, a generic theory to define structural defects is necessary for understanding the physical and mechanical behaviors of MGs. In addition, studies focusing on the MRO of amorphous alloys are still lacking, hampering our understanding of their performances. The characterization of MRO and its behavior in MGs could be a key issue to be addressed. Since the CSRO may play a similar role as the topological short range order (TSRO) in MGs, a detailed investigation of CSRO and its influences is also of fundamental importance. However, the evolution of CSRO and its correlation with MG behaviors have not been fully understood.

\subsection{Objectives and scope}

The main objective of this research work is to reveal the structure and structure-property relationship in MGs. The MD simulation is employed to reveal the underlying mechanisms of MG behaviors. The overall research scope of the PhD project is thus organized into 6 chapters. 
An overview of metallic glass, its history, structure, properties, structure-property correlation and their composites have been presented in Chapter 1. In particular, the importance of icosahedral order on the kinetics and mechanical properties is given. The motivation and objectives of the thesis have been outlined then.

Chapter 2 provides a brief overview and introduction of MD simulations. The embedded atom model has been adopted in this work to simulate the configurations of different MGs.

Chapter 3 examines the TSRO and its influences on the local dynamics and mechanical properties of MGs. The atomic level stress theory is utilized to characterize the SRO and defects in glasses and supercooled liquids. The local stress fluctuations are found to affect the local dynamic and mechanical response of glasses, which may explain the intrinsic heterogeneity of MGs.

Chapter 4 concentrates on the medium range structure of MGs and its dependence on the Voronoi index. The formation of solid-like (stable) and liquid-like (unstable) networks is intimately associated with the degree of local fivefold symmetry in $\mathrm{Zr}-\mathrm{Cu}-\mathrm{Al} \mathrm{MG}$. The correlation between atomic level stress and Voronoi index has also been revealed. In addition, the localized inelastic deformation is coupled to the medium range structure in MGs.

Chapter 5 discusses the CSRO and its influences on dynamic and mechanical heterogeneity in MGs. Similar to TSRO, the CSRO dramatically changes during supercooling, which is also dependent on the cooling histories. The correlation between CSRO and energetic stability is also presented. 
The final chapter summarizes the overall research work and outlines the topics that require further exploration. 


\section{CHAPTER 2 CLASSICAL MOLECULAR DYNAMICS}

The deformation mechanism of crystalline materials is well established by the dislocation theory, and is directly observed by electron microscopy. In contrast, due to the intrinsic amorphous structure, there is no appropriate experimental technique to examine the microscopic deformation process of MGs. On the other hand, computer simulation provides an effective alternative to explore the nanoscale phenomena in physics and materials science, such as the visualization of three dimensional configurations and explanation of structure-property relationships. However, the computation models still have serious limitations, which may lead to the disparity between the simulation and real-world materials. Among various simulation methods, MD is a straightforward method to predict the movements and trajectories of atoms following Newton's equations. According to the predetermined conditions, the physical characteristics of materials can be monitored by using the instantaneous velocity and position of the atoms.

\subsection{Applications of molecular dynamics}

In recent decades, MD simulations have been extensively used to unveil the properties of relatively large systems in terms of their atomic-level structure and the structureproperty correlations. Compared to laboratory techniques, MD simulations provide more insightful and revealing information regarding the microstructure and properties of MGs. Sheng et al. [64] investigated the atomic-level structure of various MGs by using MD simulations. The results confirm the solute-solute avoidance and strong CSRO in glasses. Additionally, the solute-to-solvent radius ratio is found to be vital in determining the dominant SRO. Due to the good GFA over a wide composition range, $\mathrm{Cu}-\mathrm{Zr}$ binary glasses have recently aroused intense interest in scientific and commercial fields. Their 
SRO and MRO have thus been resolved by several groups employing configurations obtained by MD simulations [73, 107, 108]. By conducting Voronoi analysis, clusters with high degrees of fivefold symmetry are found to be dominant in $\mathrm{Cu}-\mathrm{Zr}$. With varying compositions, the $\mathrm{CN}$ and dominant polyhedra also change gradually. In particular, the GFA of $\mathrm{Cu}-\mathrm{Zr}$ glasses are strongly related with the $\mathrm{Cu}$-centered FI with index $\langle 0,0,12,0\rangle$. Furthermore, the formation of MRO is attributed to the aggregation of $\mathrm{Cu}$-centered FI.

In addition to the structure of MGs, their properties and mechanical behaviors have been well analyzed via MD simulations. For instance, many researchers suggested the significance of icosahedral clusters on the mechanical performances of realistic $\mathrm{Cu}-\mathrm{Zr}$ based MGs $[72,80]$. The icosahedral order is found to possess higher stiffness and shear resistance, while distorted clusters correspond to lower stiffness and resistance. Cheng et al. [80] carried out a MD study to demonstrate the cooling history dependence on the deformation behaviors of $\mathrm{Cu}-\mathrm{Zr}$ MGs. In this case, the shear modulus, strength and plasticity are sensitively dependent on the cooling rates, which is consistent with experimental observations.

\subsection{Main features of molecular dynamics simulation}

This section will briefly describe the main features of MD simulation, including the initialization of atom positions and velocities, the integration techniques of atomic movements, the boundary conditions, the temperature controls and the potential functions.

Based on the Newton's second law, MD simulation can be used to predict the trajectories of particles in a classical system. If the positions and velocities of all particles at time $t$ are given, one can calculate the force on each particle and thus predict 
the acceleration and position of each atom at $t+\Delta t$ [109]. Figure 2.1 depicts a flowchart regarding the typical steps involved in the MD simulation. The initialization of a system, consisting of the initial positions and velocities of all particles, is assigned. The empirical or semi-empirical potential functions are given to determine the forces acting on those particles. Numerical integrations are then performed to obtain the positions and velocities of the particles at the succeeding moment $t+\Delta t$. The integration process is repeated until the system satisfies the stopping criteria (e.g., equilibrium state or completed loading). Quantities of the system, including configurations, temperature, pressure and etc., will be recorded during the simulation process.

A list of software programs can be used to implement MD simulations, such as Materials Studio, LAMMPS, GROMACS, NAMD. GROMACS and NAMD are designed to perform MD simulations on bio-molecular systems. They contain multiple functionality and can be executed in parallel or single processors. Materials studio is a commercial software for modeling various materials, such as polymers, metals, ceramics and so on. It can be adopted to perform $A b$ initio MD, Monte Carlo and classical MD simulations. Among all the programs, LAMMPS is an open source MD code designed for parallel computation. It contains a variety of features include the simulation of atomic, metallic and polymeric systems. In addition, LAMMPS is a developer-friendly software, since its source code is convenient to modify. Therefore, LAMMPS is adopted in this thesis to investigate the properties of MGs.

\subsubsection{Initialization}

To conduct MD simulations, firstly one needs to assign the initial configuration of all particles in a system. In the case of modeling crystalline materials, lattice parameters are required to set the accurate configuration of a system. The lattice is then repeated and 
expanded to fill the whole system. For amorphous materials, atoms can be assigned following a random allocation with the avoidance of overlapping. The initialization of atom positions can also be extracted according to experimental data. A good choice of the initial configuration is essential to obtain a simulated system similar to realistic materials. Energy minimization process is typically carried out to relief the intrinsic stress results from the initialization, and to eliminate the undesirable overlaps.

The initialization of atom velocities is also indispensable for the construction of systems. The initial atom velocities can be assigned following a uniform or Gaussian distribution in some interval. The initially assigned velocities are scaled to obtain the designated temperature. The total momentum of the system should be zero to avoid the translational drift. The numerical form is presented as

$$
\overrightarrow{P_{s y s}}=\sum_{i=1}^{N} m_{i} \vec{v}_{i}=0
$$

where $\vec{P}_{s y s}$ is the total momentum of the system, $m_{i}$ and $\vec{v}_{i}$ represent the mass and the velocity vector of atom $i, N$ is the total number of particles in the system. 


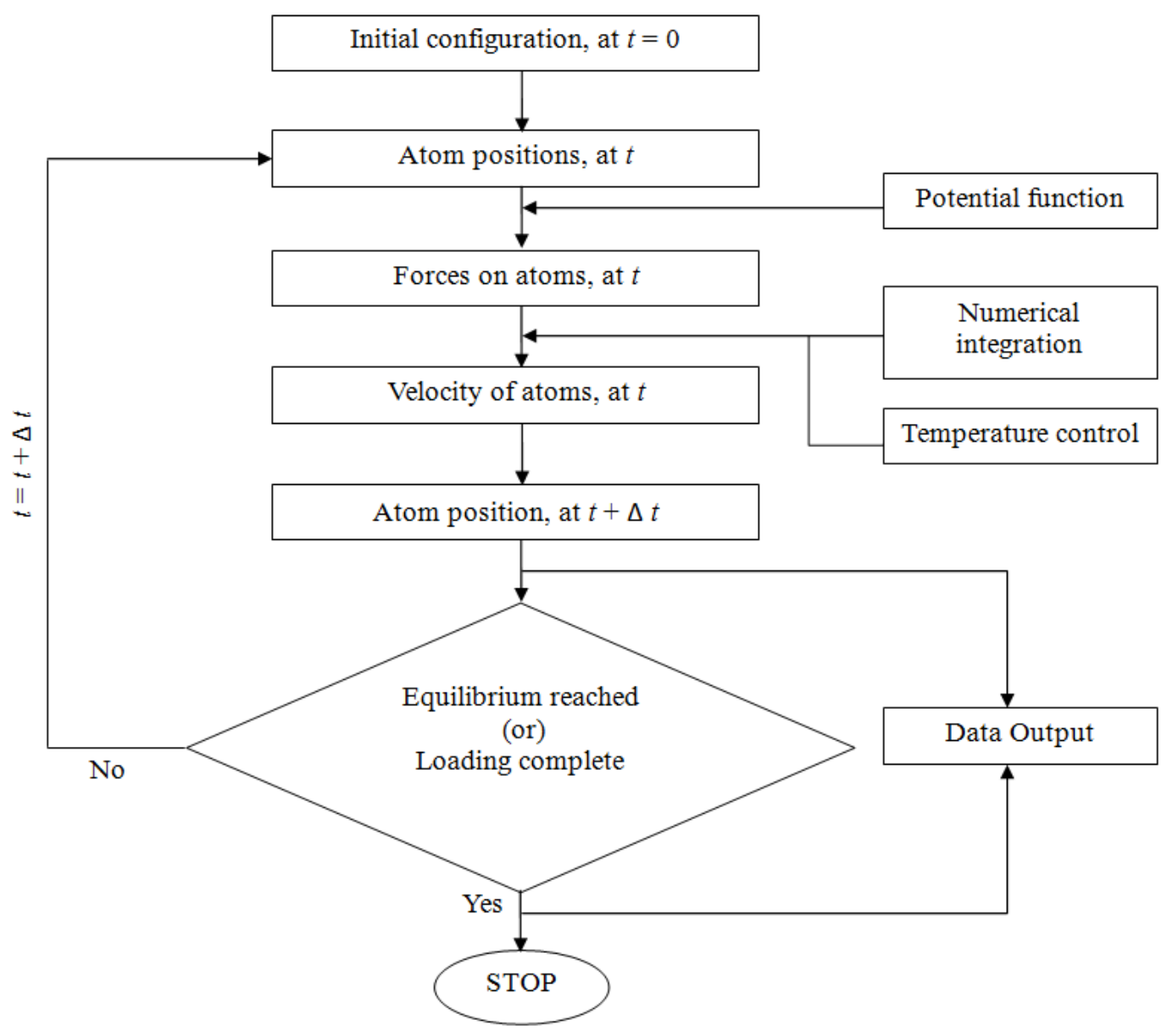

Figure 2.1 Flow chart representing the basic steps in MD simulations. 


\subsubsection{Periodic boundary conditions}

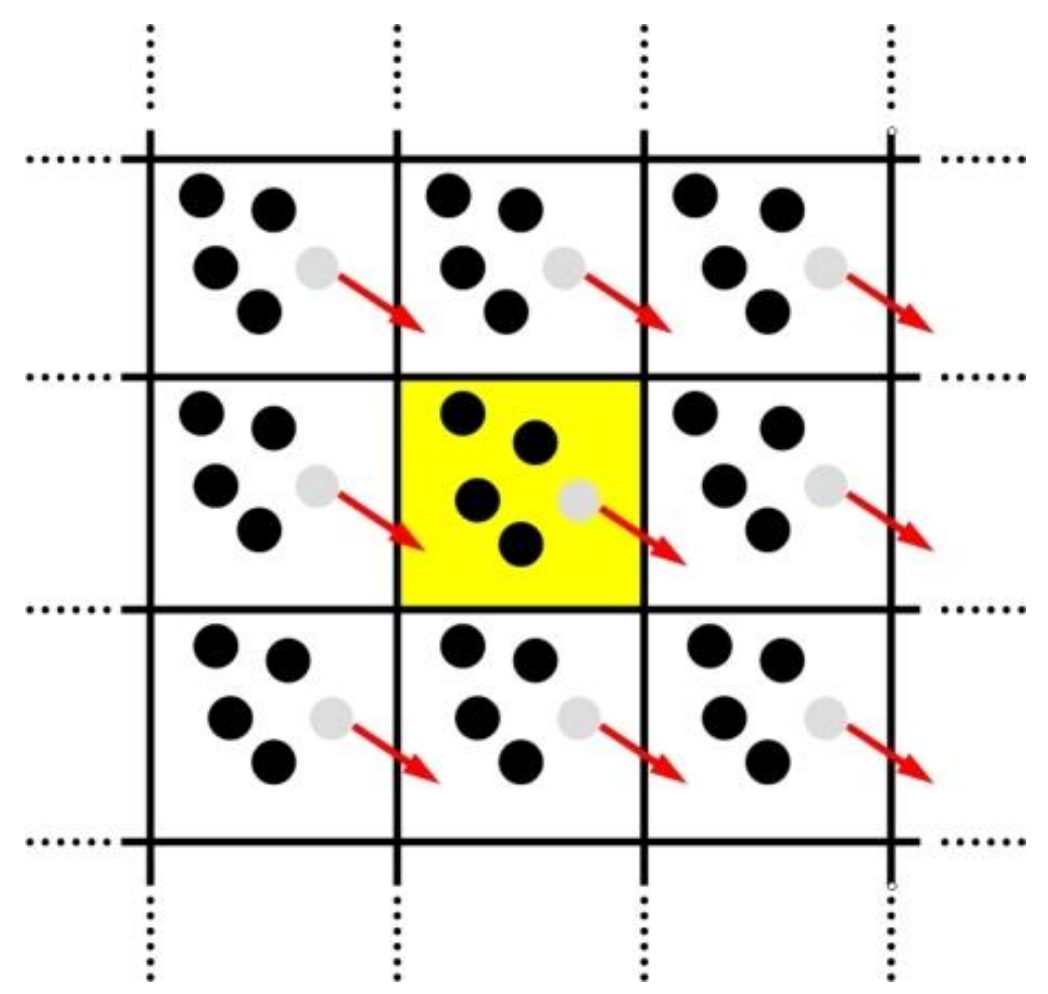

Figure 2.2 Schematic of the periodic boundary conditions adopted in a two dimensional system. The central cube represents the primary cell, and the surrounding ones are replicated images. Particles can move across the boundaries of each cube.

MD simulations are typically conducted on a small number of atoms. In general, particles near to the surface exhibit distinctly different characteristics from those inside the system. Such phenomenon is commonly named as "surface effect". The boundary conditions of a MD system could be free, fixed or periodic. The drawback of surface effect can be overcome by employing periodic boundary conditions (PBCs), which is applicable for simulating the behavior of macroscopic systems of gases, liquids and solids. Figure 2.2 shows a two dimensional system with PBCs. As shown in the figure, a primary central cell is replicated in all directions to form an infinitely periodic system. Those image cells thus share the same characteristics, such as atom number, positions and velocities. In the simulation box, as one atom in the central cell moves through a 
boundary, its images move across their corresponding boundaries. Accordingly, atoms on the surface of the primary cell share similar properties with those inside the cell. The unit cells will have to be tiled perfectly into a three dimensional crystal for the implementation of PBCs. Therefore, cubic or rectangular cells are applicable and preferred to implement PBCs in MD simulations.

\subsubsection{Temperature controls}

The instantaneous temperature of a simulated system is directly related to the kinetic energy of particles, and is given by

$$
T=\frac{M}{3 L k_{b}}\left\langle\sum_{i}^{L} \vec{v}_{i} \cdot \vec{v}_{i}\right\rangle
$$

where $T$ represents the instantaneous temperature of the system, $\vec{v}_{i}$ is the velocity of atom $i, k_{b}$ indicates the Boltzmann constant, and $L$ represents the number of the degrees of freedom.

To control the temperature of a system, the simplest method is to set the desired temperature at each timestep [110]. However, such method may cause undesired thermodynamic fluctuations in the system, which may greatly affect the behavior of the simulated system. Another straightforward method to control the system temperature is the Andersén thermostat, which is based on the reassignment of atom velocities with certain possibilities [111]. A system under Andersén thermostat is deemed coupled to a bath of stochastic impulsive forces exerted on randomly selected particles. Unfortunately, such method still presents the possibility of introducing thermodynamic perturbations. By adding damping terms into the motion equation, Berendsen, Langevin and NoséHoover thermostat can suppress the fluctuations of kinetic energy of a system [110]. The 
damping terms introduced in these schemes could be different, which determine the efficiency and accuracy of the temperature controls.

In MD simulations, an ensemble is considered as various systems with the same macroscopic properties but distinct microscopic states. Different ensemble techniques are implemented during the simulation for approximating the realistic conditions and constrains of materials. In addition, many other parameters are also assigned to suppress the energy fluctuations caused by numerical errors and etc. One commonly used ensemble in MD simulations is the micro-canonical (NVE) ensemble, which typically represents an isolated system with number of particles $N$, volume $V$ and total energy $E$ of the system being conserved. In some cases, a constant temperature is preferred for MD simulations, which requires a net input and output of energy. The constant temperature is usually fulfilled by adopting canonical (NVT) ensemble: a system with number of particles $N$, volume $V$ and temperature $T$ being conserved [112]. Another commonly used approach is the NPT ensemble, a system with fixed number of particles $N$, pressure $P$ and temperature $T$.

\subsubsection{Integration algorithms}

The trajectories of all particles during the simulation process are monitored and predicted by solving the governing equations based on Newton's second law, which is given by

$$
m_{i} \frac{d^{2} \vec{r}_{i}}{d t^{2}}=\vec{F}_{i}, \vec{F}_{i}=\frac{-\partial U}{\partial \vec{r}_{i}}
$$

where $\vec{F}_{i}$ represents the force exerted on atom $i, m_{i}$ and $\vec{r}_{i}$ indicate the mass and coordinates of atom $i, U$ is the potential energy derived from the atomic coordinates. In computer simulations, atomic movements are typically solved by numerical integrations 
with finite difference methods. The basic idea is given as follows. Given the positions and velocities of all particles at a moment $t$, their positions and velocities at a subsequent moment $t+\Delta t$ could be tracked based on the governing equations. The accuracy and efficiency of such step-by-step process are significantly dependent on the choice of integration algorithms and the time interval $\Delta t$. In general, the timestep needs to be sufficiently small to ensure the accuracy of the simulated results.

The most frequently used algorithm for integrating the motion equations is the Verlet technique, which is a direct solution of the second order equations, and given as [113]

$$
\vec{r}(t+\Delta t)=2 \vec{r}(t)-\vec{r}(t-\Delta t)+\vec{r}(t) \Delta t^{2}
$$

and

$$
\overrightarrow{\dot{r}}(t)=\frac{\vec{r}(t+\Delta t)-\vec{r}(t-\Delta t)}{2 \Delta t}
$$

As can be seen in the equations, the velocities are unnecessary for the computation of atom trajectories. The velocities are calculated so as to obtain the kinetic energy. Accordingly, the benefits of this method could be its simplicity, reversibility and memory storage. However, one of its major drawbacks is the introduction of possible numerical imperfections. The Verlet method has thus been modified and improved by a so-called "Velocity-Verlet" scheme, which gives stable results by involving the velocities in the trajectory calculations [114]. Velocities and positions at time $t+\Delta t$ are then calculated as

$$
\begin{gathered}
\overrightarrow{\dot{r}}(t+\Delta t)=\overrightarrow{\dot{r}}(t)+\frac{1}{2} \vec{r}(t+\Delta t) \Delta t+\frac{1}{2} \overrightarrow{\ddot{r}}(t) \Delta t \\
\vec{r}_{i}(t+\Delta t)=\vec{r}_{i}(t)+\overrightarrow{\dot{r}}(t) \Delta t+\frac{1}{2} \overrightarrow{\vec{r}} \Delta t^{2}
\end{gathered}
$$


The "Velocity-Verlet" algorithm is used throughout this PhD study. Hence the stability criteria of such algorithm will be discussed. The algorithmic stability depends both on the numerical scheme and the differential equations being solved. In molecular dynamics simulation, a simple harmonic oscillator (spring-mass system) is typically used to determine the stability of the numerical schemes. For this "Velocity-Verlet" method, we must have $0<\omega \Delta t<2$ to provide stable solutions to the harmonic oscillator problem. $\omega$ represents the natural frequency of the harmonic oscillator, and $\Delta t$ denotes the timestep. The typical order of atomic vibration is $10^{13}$ to $10^{14} \mathrm{~Hz}$. Therefore, a vibrational period should be split into several segments for MD to satisfy the numerical stability. Accordingly, 1 fs $\left(10^{-15} \mathrm{~s}\right)$ is used to simulate the behaviors of metallic materials.

Another alternative algorithm in MD simulations is the predictor-corrector technique, consisting of three steps: prediction, evaluation and correction. Firstly, positions and velocities of all particles at time $t+\Delta t$ are predicted based on the information at time $t$. After that, the predicted and evaluated values are compared to correct the results. For atom $i$, its position $\vec{r}_{i}$ and corresponding derivatives, $\overrightarrow{\dot{r}}_{i}, \vec{r}_{i}, \vec{r}_{i}, \vec{r}_{i}^{(i v)}$, and $\vec{r}_{i}^{(v)}$, at the moment $t+\Delta t$ are predicted based on Taylor's expansion, which are described as

$$
\begin{gathered}
\vec{r}_{i}^{P}(t+\Delta t)=\vec{r}_{i}(t)+\overrightarrow{\dot{r}}_{i}(t) \Delta t+\overrightarrow{\vec{r}}_{i}(t) \frac{(\Delta t)^{2}}{2 !}+\overrightarrow{\ddot{r}}_{i}(t) \frac{(\Delta t)^{3}}{3 !}+\vec{r}_{i}^{(i v)}(t) \frac{(\Delta t)_{4}}{4 !}+\vec{r}_{i}^{(v)}(t) \frac{(\Delta t)^{5}}{5 !} \\
\overrightarrow{\dot{r}}_{i}^{P}(t+\Delta t)=\overrightarrow{\dot{r}}_{i}(t)+\overrightarrow{\ddot{r}}_{i}(t) \Delta t+\overrightarrow{\ddot{r}}_{i}(t) \frac{(\Delta t)^{2}}{2 !}+\vec{r}_{i}^{(i v)}(t) \frac{(\Delta t)^{3}}{3 !}+\vec{r}_{i}^{(v)}(t) \frac{(\Delta t)^{4}}{4 !} \\
\overrightarrow{\vec{r}}_{i}^{P}(t+\Delta t)=\overrightarrow{\vec{r}}_{i}(t)+\overrightarrow{\ddot{r}}_{i}(t) \Delta t+\vec{r}_{i}^{(i v)}(t) \frac{(\Delta t)^{2}}{2 !}+\vec{r}_{i}^{(v)}(t) \frac{(\Delta t)^{3}}{3 !} \\
\overrightarrow{\ddot{r}}_{i}^{P}(t+\Delta t)=\vec{r}_{i}(t)+\vec{r}_{i}^{(i v)}(t) \Delta t+\vec{r}_{i}^{(v)}(t) \frac{(\Delta t)^{2}}{2 !}
\end{gathered}
$$




$$
\begin{gathered}
\vec{r}_{i}^{(i v)^{P}}(t+\Delta t)=\vec{r}_{i}^{(i v)}(t)+\vec{r}_{i}^{(v)}(t) \Delta t \\
\vec{r}_{i}^{(v)^{P}}(t+\Delta t)=\vec{r}_{i}^{(v)}(t) \Delta t
\end{gathered}
$$

Based on the predicted positions, the force $\vec{F}_{i}(t+\Delta t)$ and acceleration $\vec{a}_{i}{ }^{E}(t+\Delta t)$ could be evaluated, which is adopted as a corrector. The difference between the predicted and the evaluated accelerations is calculated as

$$
\Delta \overrightarrow{\ddot{r}}_{i}=\left[\Delta \overrightarrow{\ddot{r}}_{i}^{E}(t+\Delta t)-\overrightarrow{\vec{r}}_{i}^{P}(t+\Delta t)\right]
$$

This term is used as a corrector to modify the predicted positions and those derivatives. The modification process is described as following equations

$$
\begin{gathered}
\vec{r}_{i}=\vec{r}_{i}^{P}+\frac{3}{16}\left(\Delta R_{2}\right) \\
\overrightarrow{\dot{r}}_{i}=\overrightarrow{\dot{r}}_{i}^{P}+\frac{251}{360}\left(\Delta R_{2}\right) \\
\overrightarrow{\vec{r}}_{i} \frac{(\Delta t)^{2}}{2 !}={\overrightarrow{\vec{r}_{i}}}^{P} \frac{(\Delta t)^{2}}{2 !}+\left(\Delta R_{2}\right) \\
\overrightarrow{\ddot{r}}_{i} \frac{(\Delta t)^{3}}{3 !}=\overrightarrow{\ddot{r}}_{i}^{P} \frac{(\Delta t)^{3}}{3 !}+\frac{11}{18}\left(\Delta R_{2}\right) \\
\vec{r}_{i}^{(i v)} \frac{(\Delta t)^{4}}{4 !}=\vec{r}_{i}^{(i v) P} \frac{(\Delta t)^{4}}{4 !}+\frac{1}{6}\left(\Delta R_{2}\right) \\
\vec{r}_{i}^{(i v)} \frac{(\Delta t)^{5}}{5 !}=r_{i}^{(v) P} \frac{(\Delta t)^{5}}{5 !}+\frac{\left(\Delta R_{2}\right)}{60}
\end{gathered}
$$

where 


$$
\Delta R_{2}=\frac{\Delta \overrightarrow{\ddot{r}}_{i}(\Delta t)^{2}}{2}
$$

In general, the predictor-corrector algorithm provides more accurate and stable results than Verlet algorithm. However, its applications are sometimes limited due to the time and memory-consuming characteristics.

\subsubsection{Potential models}

The focus of MD simulation involves the calculation of potential energy of a particular model and forces acting on all particles. The accurate form of potential function could be derived from the quantum mechanics with solving the Schrödinger's equation. This approach is named as ab initio MD or first-principle MD, which could describe the electronic structure and interatomic interactions with accuracy. Unfortunately, ab initio MD is limited to the modeling of small system due to the expensive computational cost. Therefore, empirical or semi-empirical potentials have been developed to alleviate the difficulties of $a b$ initio MD. Typically, the empirical potentials could be developed by fitting the potential parameters with the experimental results and $a b$ initio data. In classical MD simulations, various potential functions are developed to implement certain particular system of interest. The most commonly used potential function is the LennardJones (LJ) potential, and the potential energy between two particles is given by [109]

$$
V(r)=4 \varepsilon\left[\left(\frac{\sigma}{r}\right)^{12}-\left(\frac{\sigma}{r}\right)^{6}\right]
$$

where $\sigma$ represents the equilibrium interatomic distance, $r$ is the distance between two atoms, and $\varepsilon$ quantifies the depth of the potential. The simple expression of LJ potential is useful for ultrafast calculation, and it is found suitable for modeling inert gases or systems with van der Waals interactions. However, the LJ potential is typically 
inaccurate for simulating metallic materials. Therefore, many-body interaction potentials, such as embedded atom model (EAM) potentials [115], were developed to suppress the drawbacks. The EAM potential is particularly appropriate for modeling the metallic materials, due to the non-directional bonds between metallic elements and the relatively simple and fast computation. Therefore the EAM formula is commonly implemented in the MD simulations of MGs. The potential energy of an atom in EAM can be expressed as [71]

$$
E_{i}=F_{\alpha}\left(\sum_{i \neq j} \rho_{\beta}\left(r_{i j}\right)\right)+\frac{1}{2} \sum_{i \neq j} \phi_{\alpha \beta}\left(r_{i j}\right)
$$

where $r_{i j}$ is the distance between atom $i$ and $j, \phi_{\alpha \beta}$ is the pair potential function between element types $\alpha$ and $\beta, \rho_{\beta}$ is the charge density of $\beta$, and $F_{\alpha}$ represents the embedding function. The total potential energy of the whole system is the sum of the potential energy of individual atom.

\subsubsection{Limitations of MD simulations}

In addition to experiments, computer simulation is used as an alternative to study the structure of metallic glasses (MGs). However, the main limitation of MD simulations is their time and spatial scales. The time scale of classical MD can reach tens of nanoseconds, and the length scale is on the order of $100 \mathrm{~nm}$. The nanosecond time scale of MD simulations is significantly shorter than the timescale for real-world $\mathrm{MG}$ formation. Therefore, one must keep in mind the large discrepancy between simulated and realistic MGs. Despite such limitations, MD is still a powerful tool to investigate the atomic-level structure and underlying mechanisms of MGs.

The embedded atom method (EAM) potential is employed to mimic the atomic force field of realistic MGs. The angular dependence is ignored in the EAM potential, 
resulting in the inaccuracy of some systems. Due to the non-directional feature of metallic bonds, such simplification is particularly suitable for modeling metallic materials. The simplicity of EAM interactions makes the computations relatively fast. Some empirical potentials including the angular dependence can also be used to simulate MGs. However, the computation speed is significantly slower than the EAM potential.

\subsubsection{Data output}

MD simulations are commonly used to simulate the realistic processes in materials in microscopic scale. By tracking and post-processing the atom trajectories and velocities during the MD simulations, various parameters could be calculated to investigate different properties and characteristics of materials, such as strength, failure, plastic behavior etc. For instance, the mechanical behaviors of materials could be monitored by applying different loadings to samples and tracking the atomic displacements. Other sophisticated parameters such as thermal conductivity could be derived by postprocessing the simulated results. Typically, the thermodynamic parameters (i.e., temperature, pressure and total energy) reflect the macroscopic properties of materials, whereas the fluctuations of microscopic states (i.e., local stiffness and atomic level structure) are studied based on the information in terms of individual atoms. 


\section{CHAPTER 3 SHORT-RANGE ORDER AND DEFECTS IN}

\section{METALLIC GLASSES STUDIED BY ATOMIC LEVEL STRESS}

\section{THEORY}

By using MD simulations, this chapter provides an insight into the structure-property relationship in $\mathrm{Cu}-\mathrm{Zr}$ MGs based on the atomic level stress theory. After various MG samples were prepared, the atomic level stress on each atom was computed and correlated with the local dynamic and mechanical behaviors in MGs. Specifically, the mean square displacement (MSD) and von Mises shear strain $\left(\eta_{i}^{\text {Mises }}\right)$ were calculated to track the inhomogeneous distribution of atomic mobility and local stiffness in MGs.

\subsection{Introduction}

One of the fundamental interests in MGs is the microscopic mechanisms underlying their physical and mechanical properties [49, 116, 117]. Conventionally, the structural defects in materials (e.g., dislocations in crystals) play a vital role in determining the dynamic and mechanical behaviors. Unfortunately, due to the blurry boundary between structural order and defects, knowledge on the structural defects in MGs has not been well established [118]. Therefore at present, there is a pressing need for the clear definition and understanding of structural defects in glasses.

In addition to experimental techniques, MD simulation provides an alternative to unambiguously study the nanoscale structure and structure-property correlations in materials [70, 119]. Specifically, the Voronoi index is extensively adopted to differentiate structural order and defects in MGs [64]. For example, numerous computer simulations confirmed the stability of $\langle 0,0,12,0\rangle$ full icosahedra (FI) in $\mathrm{Cu}-\mathrm{Zr}$ MGs 
[120-122]. Peng et al. [106, 123] analyzed the structure-dynamics relationship in $\mathrm{Cu}-\mathrm{Zr}$ glasses via classical MD simulations. They reported that some $\mathrm{Zr}$-centered clusters, e.g. $\langle 0,1,10,4\rangle$ and $\langle 0,1,10,5\rangle$, fundamentally determine the slow dynamics in all compositions. Additionally, $\mathrm{Cu}-$-centered $\langle 0,0,12,0\rangle$ FI exhibit slow kinetics at $\mathrm{Cu}$-rich alloys. Cheng et al. [80] modeled the shear transformation process in $\mathrm{Cu}_{64} \mathrm{Zr}_{36}$ MG. The results revealed that FI exhibit high stability and resistance to local shear deformation, while the low-population clusters serve as fertile sites for ST events. However, such criterion is limited to particular types of glass-forming systems, since the dominant Voronoi clusters change with alloy systems and compositions [124-126]. Accordingly, a system-independent criterion to generally define and understand the structural defects in amorphous materials is needed.

Another approach to identify structural defects in MGs is the atomic-level stress theory proposed by Egami et al. $[66,127]$. Typically, the local equilibrium of an atom can be characterized by the zero stress state, while the deviations can be reflected by the atomic-level stresses [127]. Atoms with excessive stresses are hence unstable and defined as topological defects. For example, $n$-type (negative density fluctuation) and $p$-type defects (positive density fluctuation) are characterized according to the local volume strain, which is associated with the atomic hydrostatic pressure. According to Egami's criterion, $n$-type defects are atoms with more than $11 \%$ local volume expansion, whereas $p$-type defects are atoms with more than $11 \%$ local volume compression. Additionally, $\gamma$-defects are considered as atoms with excessive von Mises shear stresses, which are assumed to be coupled to the shear localization phenomenon in MGs. Recently, the suitability of such theory has been proved by Cheng et al. [118]. The atomic-level pressure is closely correlated with some topological parameters in the 
simulated samples. It is suggested that atoms with low strain energy are preferentially to form regular clusters, such as $\langle 0,0,12,0\rangle$ in $\mathrm{Cu}_{64} \mathrm{Zr}_{36}$ and $\langle 0,3,6,0\rangle$ in $\mathrm{Pd}_{82} \mathrm{Si}_{18}$. Accordingly, atomic-level stress distributions may also affect the local dynamic and mechanical properties of MGs. So far, however, few studies have addressed related issues [127].

In this chapter, MD simulations with EAM potentials [71] were implemented to determine the properties of $\mathrm{Cu}_{\mathrm{x}} \mathrm{Zr}_{100-\mathrm{x}}$ (where $\mathrm{x}=35,50$ or 65) MGs. On the basis of these simulations, MSDs and von Mises shear strain were extracted to describe the local kinetic and mechanical features of these glassy samples. The results reveal the significance of atomic-level stresses in determining the local properties of MGs. Atoms with high von Mises shear stress ( $\gamma$-defects) exhibit faster dynamics in all compositions. In addition, it is demonstrated here that $\gamma$-defects are more prone to experience local plastic deformation than the solid-like matrix.

\subsection{Simulation methods}

Large-scale atomic/molecular massively parallel simulator (LAMMPS) [128] was adopted to simulate $\mathrm{Cu}_{x} \mathrm{Zr}_{100-x}$ (where $\mathrm{x}=35,50$ or 65) glasses containing 16,000 atoms. EAM potentials were employed to determine the interatomic forces in our models [71]. The molecular systems, with periodic boundary conditions applied in all axial directions, were firstly melted and equilibrated at $2000 \mathrm{~K}$ for $2 \mathrm{~ns}$. The samples were then quenched rapidly from the liquid states to glassy states $(50 \mathrm{~K})$ at a cooling rate of $5 \times 10^{11} \mathrm{~K} / \mathrm{s}$. After cooling, the systems were equilibrated for another $2 \mathrm{~ns}$. In the simulations, the time step was set at 1 fs and isothermal isobaric (NPT) ensemble were employed to control the temperature and pressure. The structural configurations for each composition at $T=800$ $\mathrm{K}$ and $50 \mathrm{~K}$ were collected to analyze the annealing $(800 \mathrm{~K})$ and the shear deformation 
processes $(50 \mathrm{~K})$ respectively. A typical $3 \mathrm{D}$ configuration of $\mathrm{Cu}_{50} \mathrm{Zr}_{50} \mathrm{MG}$ is shown in Figure 3.1(a).

(a)

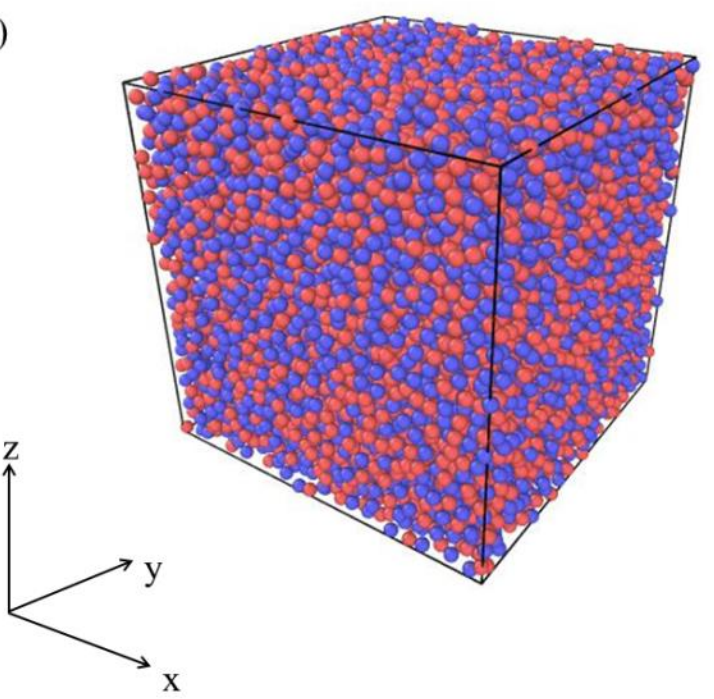

(b)

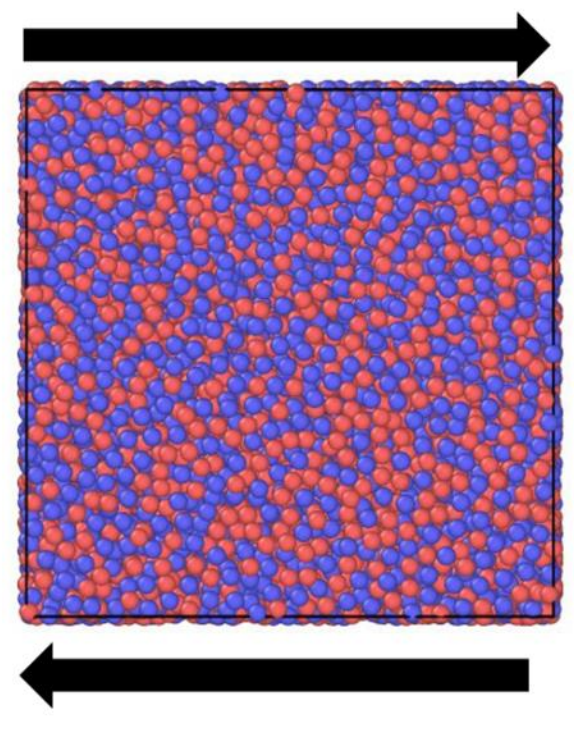

Figure 3.1 (a) 3D Configuration of the simulated $\mathrm{Cu}_{50} \mathrm{Zr}_{50}$ MG. (b) Schematic of shear loading on a $\mathrm{Cu}_{50} \mathrm{Zr}_{50} \mathrm{MG}$ by using supercell tilting.

To investigate the local dynamic properties of MGs, rapid quenching processes from $2000 \mathrm{~K}$ to $800 \mathrm{~K}$ were implemented at $5 \times 10^{11} \mathrm{~K} / \mathrm{s}$ under NPT ensemble for three 
different compositions. After cooling, these systems were kept at $800 \mathrm{~K}$ to model the annealing process at constant temperature. The configurations and atom coordinates were collected over the simulation duration for structural and kinetic analyses. In addition, shear deformation was simulated at $50 \mathrm{~K}$ to probe the local mechanical behaviors of $\mathrm{Cu}-\mathrm{Zr}$ MGs. Figure 3.1(b) shows a schematic representation of pure shear loading of the $\mathrm{Cu}_{50} \mathrm{Zr}_{50}$ sample at $50 \mathrm{~K}$ with PBCs. The samples were deformed by supercell tilting followed by MD relaxation, with a strain rate of $10^{9} \mathrm{~s}^{-1}$. The low temperature $(50 \mathrm{~K})$ was adopted here to eliminate the effects of thermal relaxation, as simulated MGs are usually more prone to thermal activations than real-world glasses [80].

After recording the 3D configurations of the amorphous samples, radial distribution functions (RDFs) were computed to prove the reliability of the simulated models. The partial RDFs are defined as [110]

$$
g_{\alpha \beta}(r)=\frac{V}{N_{\alpha} N_{\beta}}\left\langle\sum_{i}^{N} \sum_{i \neq j}^{N} \delta\left(r-r_{i j}\right)\right\rangle
$$

where $V$ represents the total volume of the system, $N_{\alpha}$ and $N_{\beta}$ are the numbers of atoms of type $\alpha$ and type $\beta$, respectively. $r_{i j}$ is the interatomic distance between atom $i$ and $j, \delta$ is the dirac delta function, and the angular brackets indicate time average.

The local environment for each atom is reflected by the atomic-level stresses. Egami and collaborators [66] proposed this concept to demonstrate the local topological instability in MGs. The six components of atomic-level stresses could be directly derived from the pair potentials. In this work von Mises shear stress was computed to study the dynamic and mechanical features of structural defects. The von Mises shear stress on each atom is computed as [127] 


$$
\gamma_{i}=\left[\frac{1}{3}\left\{\frac{\left(\sigma_{i}^{1}-\sigma_{i}^{2}\right)^{2}}{2}+\frac{\left(\sigma_{i}^{2}-\sigma_{i}^{3}\right)^{2}}{2}+\frac{\left(\sigma_{i}^{1}-\sigma_{i}^{3}\right)^{2}}{2}\right\}\right]^{\frac{1}{2}}
$$

where $\gamma_{i}$ is the von Mises shear stress on atom $i, \sigma_{i}^{1}, \sigma_{i}^{2}$ and $\sigma_{i}^{3}$ are the three principal stresses.

Mean square displacements (MSDs) can be used to investigate the local kinetics of structural defects in MGs. For instance, the MSDs are usually employed to calculate the diffusivity of materials. The MSD is defined as

$$
\operatorname{MSD}=\left\langle\left|r_{i}(\mathrm{t})-r_{i}(0)\right|^{2}\right\rangle
$$

where $r_{i}(0)$ and $r_{i}(\mathrm{t})$ respectively represent the position of atom $i$ at the moment 0 and $t$, and the angular brackets represent an average on the particles.

In order to examine the shear localization in MGs, von Mises shear strain for each atom was monitored during the shear deformation processes. Similar to the $D_{\min }^{2}$ proposed by Falk and Langer [129], this parameter is a good measurement of local inelastic deformation. The local shear invariant can be computed as [130]

$$
\eta_{i}^{\text {Mises }}=\sqrt{\eta_{y z}^{2}+\eta_{x z}^{2}+\eta_{x y}^{2}+\frac{\left(\eta_{y y}^{2}-\eta_{z z}^{2}\right)+\left(\eta_{y y}^{2}-\eta_{x x}^{2}\right)+\left(\eta_{x x}^{2}-\eta_{z z}^{2}\right)}{6}}
$$

details on this parameter could be found in ref [130].

\subsection{Results and discussion}

\subsubsection{Radial distribution functions}

RDFs are extensively used to verify the reliability of simulation results. The comparison of the simulated and experimental RDFs is shown in Figure 3.2. The experimental RDFs 
calculated by neutron diffraction (ND) approach were taken from Ref. [131]. After taking into consideration the different cooling conditions, the three RDFs of the modeled glasses exhibit remarkable agreement with the experimental values, including the shape, magnitude and peak location. This consistency validates the implementation of MD to explore the structure and properties of the real-world $\mathrm{Cu}-\mathrm{Zr}$ MGs.

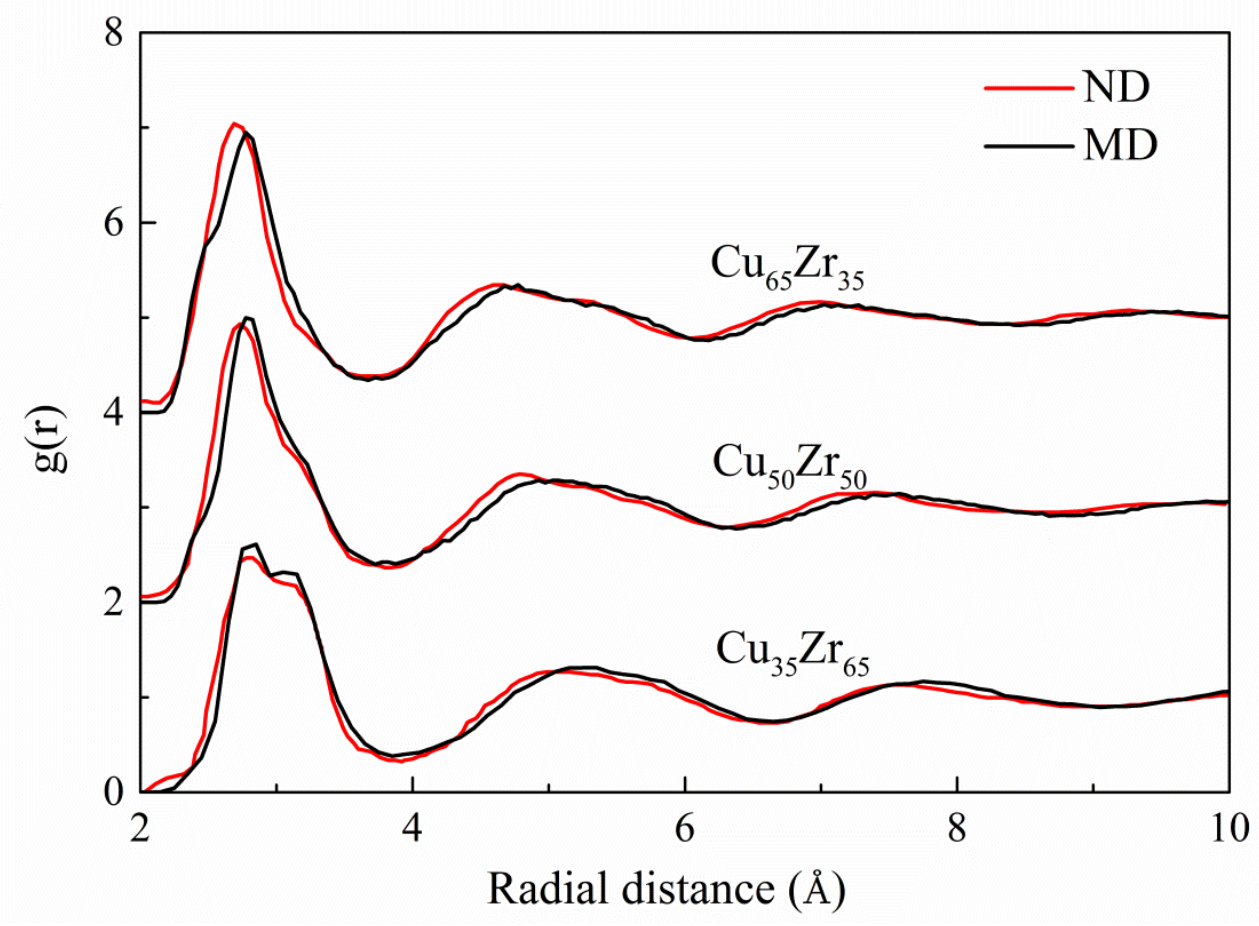

Figure 3.2 Comparison between RDFs obtained by experiment [131] and MD simulation in three different compositions of $\mathrm{Cu}_{\mathrm{x}} \mathrm{Zr}_{100-\mathrm{x}}$ MGs. Individual curves are shifted vertically for clarity.

\subsubsection{Atomic-level stress distribution}

In this chapter, the structural defects in $\mathrm{Cu}-\mathrm{Zr}$ glasses are characterized by the different level of atomic-level stresses. Naturally, the origin of atomic-level stresses is attributed to the frustrations between the equilibrium state and the local distorted environment [66]. Therefore atoms with excessive stresses are expected to exhibit instability under external 
stimuli, such as thermal perturbations and applied loads. According to Egami's theory, there exist three types of structural defects in MGs: namely $n$-type, $p$-type and $\gamma$-type. Typically, $n$-type and $p$-type defects are directly associated with the atomic-level pressure (or local density), whereas $\gamma$-defects are characterized by the values of von Mises shear stress. Here we mainly discuss the characteristics of atoms with high shear stresses ( $\gamma$-defects), since this parameter is found to be closely related to the local dynamic and mechanical properties of MGs. In this study, atoms of the same species were sorted out according to the magnitude of atomic shear stress (from low to high). $\gamma$-defects are defined as those atoms with highest atomic shear stress.

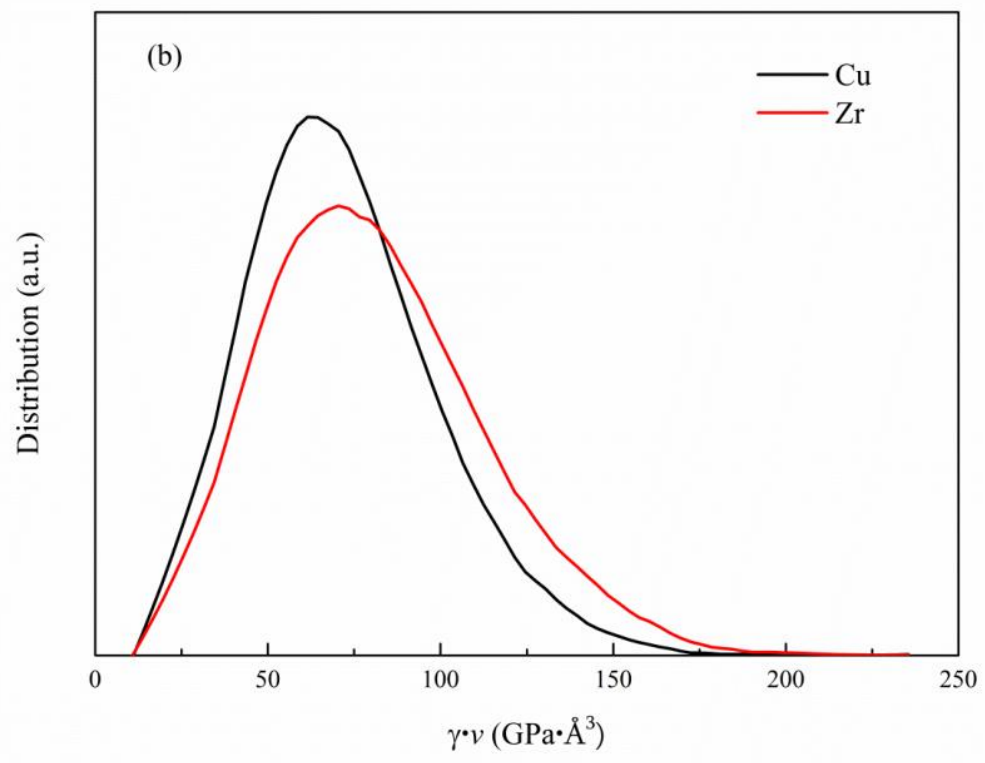

Figure 3.3 Distribution of von Mises shear stress multiplied by atomic volume in $\mathrm{Cu}_{50} \mathrm{Zr}_{50} \mathrm{MG}$.

Figure 3.3 denotes the atomic shear stress distribution in $\mathrm{Cu}_{50} \mathrm{Zr}_{50} \mathrm{MG}$. As shown in the figure, the distribution diagram is non-Gaussian like and highly heterogeneous. According to Egami and other researchers $[66,118]$, atoms with highest shear stress are liquid-like and topologically unstable, while the solid-like matrix is constructed by 
those low-stress atoms. The distributions of atomic shear stress for other two compositions are also similar (not shown here). Interestingly, with $\mathrm{Cu}$ concentration increasing (from $x=35$ to 65 ), we notice that the average shear stress on $\mathrm{Cu}$ atoms decreases. The average shear stresses on $\mathrm{Cu}$ atoms for $\mathrm{Cu}_{35} \mathrm{Zr}_{65}, \mathrm{Cu}_{50} \mathrm{Zr}_{50}, \mathrm{Cu}_{65} \mathrm{Zr}_{35}$ are 73.26, 72.35 and $70.04 \mathrm{GPa} \cdot \AA^{3}$ respectively. Such trend may be coupled to the formation of $\langle 0,0,12,0\rangle$ FI, since many studies reported the ease of FI formation at $\mathrm{Cu}$-rich compositions [120]. The kinetic and mechanical properties of atoms with highest shear stress ( $\gamma$-defects) are mainly explored, as discussed in following sections.

\subsubsection{Influences of atomic shear stress on local dynamics}

In many atomistic simulations, annealing processes at a constant temperature have been carried out to probe the dynamic heterogeneity of MGs [124]. For instance, Peng et al. [106] proposed the strong correlations between Voronoi indices and MSDs in $\mathrm{Cu}-\mathrm{Zr}$ glasses by modeling annealing processes. The results proved that the Voronoi index has significant influence on local kinetics and glass forming ability (GFA) of MGs. To study the influences of atomic level shear stress, we modeled the annealing processes of $\mathrm{Cu}-\mathrm{Zr}$ MGs at $800 \mathrm{~K}$ and collected the atomic coordinates. The annealing temperature selected here is slightly above the reported glass transition temperatures of $\mathrm{Cu}-\mathrm{Zr}$ MGs [73], to ensure that the atomic mobility is relatively high at such temperature. In this section, the $25 \%$ atoms with the highest von Mises shear stress are regarded as $\gamma$-defects. To monitor the correlations between the atomic shear stress and local kinetics, the movement of atoms was tracked during the annealing processes.

MSD is usually considered as an indicator to probe the local kinetics variations in amorphous alloys, since it unambiguously reveals how fast atoms are transported in supercooled liquids. In our simulations, The non-Gaussian parameter (NGP) was firstly 
computed to acquire the "maximum non-Gaussian time", which denotes the time at which the NGP reaches the maximum. The "maximum non-Gaussian time" is typically adopted to study the dynamic heterogeneity in supercooled liquids. Such parameter represents the time when the distribution of atomic motion becomes the most heterogeneous, which corresponds to the time spot right before the $\alpha$-relaxation. The NGP can be computed as

$$
\alpha_{2}(t)=\frac{3\left\langle\left|r_{i}(t)-r_{i}(0)\right|^{4}\right\rangle}{5\left\langle\left|r_{i}(t)-r_{i}(0)\right|^{2}\right\rangle^{2}}-1
$$

In this study, the time interval to study the dynamic heterogeneity was chosen to be 20 ps (maximum non-Gaussian time). The local dynamics was then evaluated by monitoring the atomic displacement for this time interval. 500 simulation runs were performed and averaged, with the same initial configuration but different distribution of initial momenta of atoms. By averaging the MSDs, the effect of initial momenta distribution is eliminated, and atomic mobility can be directly related with the von Mises shear stress. The correlation between local dynamics and $\gamma$-defects is shown in Figures 3.4 (a) to (c). In the figures, $\mathrm{Cu}$ and $\mathrm{Zr}$ atoms were firstly sorted by their displacement from low to high. Then the atoms were divided into 10 groups, each containing $10 \%$ of the total $\mathrm{Cu}$ or $\mathrm{Zr}$ atoms. The fraction of $\gamma$-defects was then computed within each group, as illustrated by the histogram. The left and right ends respectively represent the slowest and fastest groups. The fraction indicates whether $\gamma$-defects could be correlated with the distribution of local kinetics. 


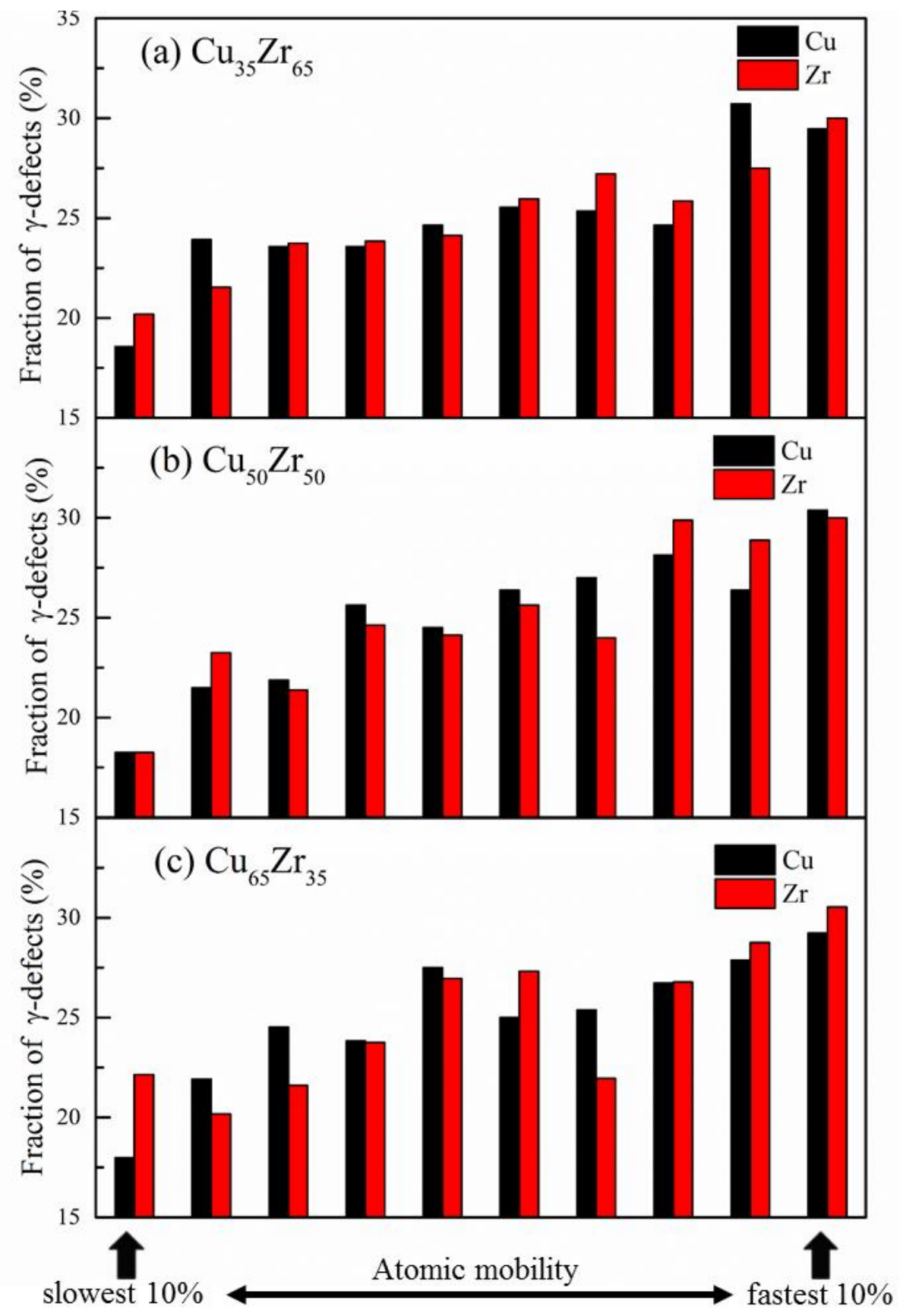

Figure 3.4 Correlations between $\gamma$-defects and atomic mobility in $\mathrm{Cu}_{x} \mathrm{Zr}_{100-x}$ glass forming liquids. $\mathrm{Cu}$ and $\mathrm{Zr}$ atoms are sorted by their displacement from low to high. Atoms are then divided into 10 groups, each containing $10 \%$ of the total $\mathrm{Cu}$ or $\mathrm{Zr}$ atoms. The left and right end represent the $10 \%$ atoms with the lowest and highest mobility, respectively. The histogram shows the fraction of $\gamma$-defects in each group. 
As shown in Figures 3.4 (a) to (c), the lower the atomic mobility, the lower the concentration of $\gamma$-defects. In all compositions, it can be seen that $\gamma$-defects tend to exhibit faster dynamics during the annealing processes, and hence can be regarded as liquid-like atoms in glass forming liquids. Such finding indicates the universal correlation between atomic shear stress and local kinetics in $\mathrm{Cu}-\mathrm{Zr}$ supercooled liquids. During the annealing processes, $\gamma$-defects are easier to be activated, showing faster dynamics than those low-stress atoms. Such finding can be explained by the local activations of atoms for structural relaxations. As proposed by Spaepen et al.[48], activations or rearrangements of atoms in supercooled liquids are accompanied with the overcoming of energy barriers. At low temperature, atoms are mostly encaged by their surrounding neighbors, due to the insurmountable energy barriers (high viscosity) in glasses. With external stimuli (e.g., thermal perturbations or applied loads), high-stress atoms are prone to be activated and transformed into low-stress (stable) states. This may explain why unfavorable atoms ( $\gamma$-defects in this study) generally exhibit faster dynamics during the annealing processes. The fluctuations of atomic level stress account for the inhomogeneous distribution of local dynamics in glass forming liquids.

Compared to the Voronoi index, atomic-level shear stress is proved to be a systemindependent parameter to characterize the structural defects in glass forming liquids, especially suitable for those icosahedra-poor glasses (e.g., $\mathrm{Cu}_{35} \mathrm{Zr}_{65} \mathrm{MG}$ ). Based on the results, we found that atomic shear stress distribution fundamentally determines the local dynamic properties of MGs.

\subsubsection{Influence of atomic shear stress on local mechanical responses}

The Achilles heel of BMGs is their low-temperature brittleness, which is commonly considered to stem from the formation of localized shear bands. A comprehensive 
understanding of shear localization mechanism is therefore helpful for researchers to develop BMG formers with improved plasticity. For instance, FI are believed to exhibit high stability and shear resistance in $\mathrm{Cu}_{65} \mathrm{Zr}_{35}$ glass, while loosely packed clusters tend to undergo plastic deformation under external loads [120]. This section examines the general dependence of von Mises shear stress on local mechanical responses of $\mathrm{Cu}-\mathrm{Zr}$ glasses. 

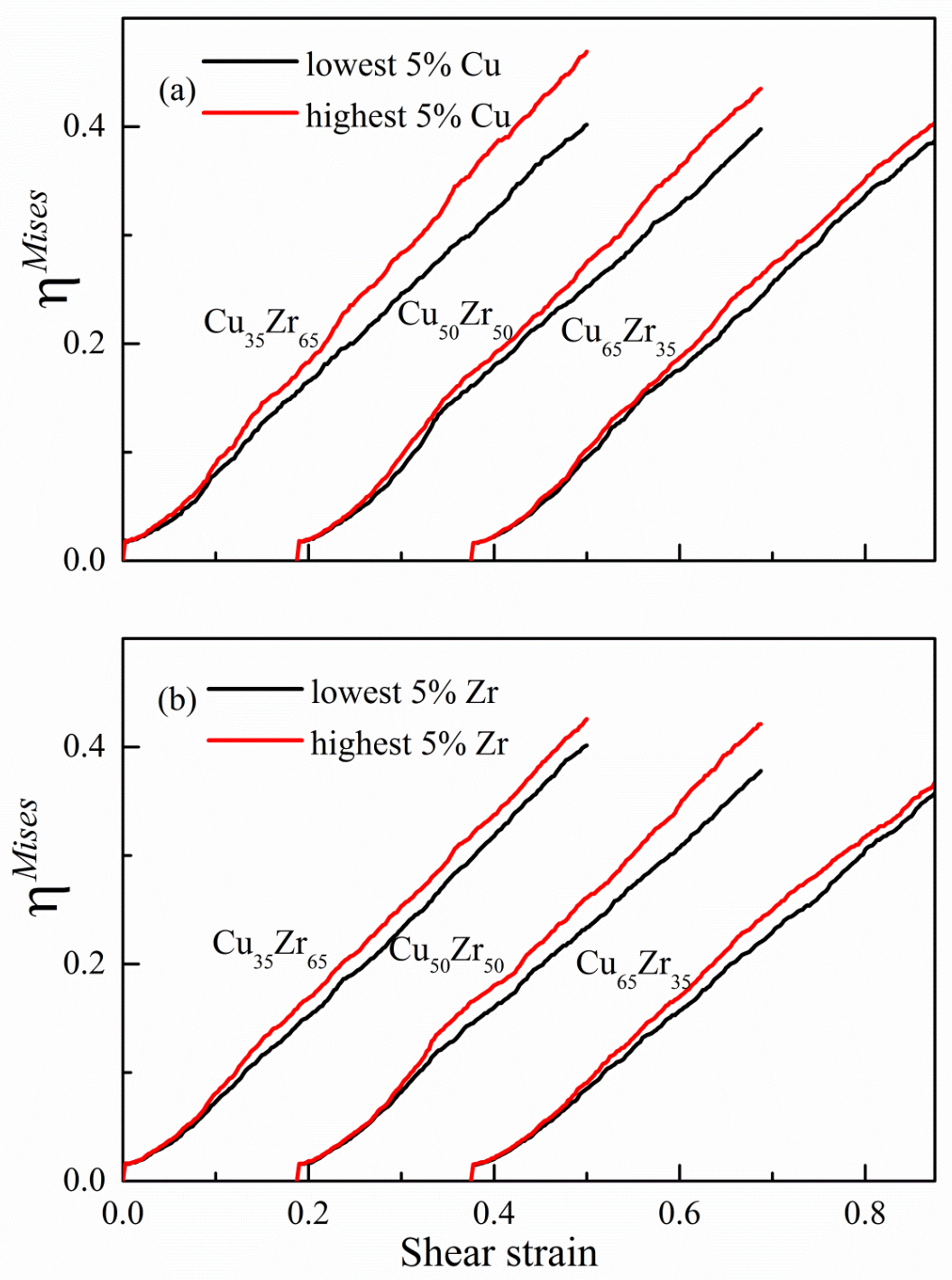

Figure 3.5 The average atomic shear strain evolutions of $\gamma$-defects and solid-like atoms for (a) $\mathrm{Cu}$ and (b) $\mathrm{Zr}$ atoms during shear deformation in three different compositions. Individual curves are shifted horizontally for clarity.

Like Falk and Langer's $D_{\min }^{2}$ [129], atomic local shear strain $\eta_{i}^{\text {Mises }}$ can be extracted to characterize the local plastic transformation at the atomic level. Atomic coordinates were collected during the simulated shearing processes to monitor the $\eta_{i}^{\text {Mises }}$ for each 
atom. We sorted out atoms of the same species according to the magnitude of the von Mises shear stress (from low to high). According to Egami's definition, atoms with excessive shear stresses are regarded as $\gamma$-defects in glasses, whereas atoms with low shear stress are stable and solid-like. To identify the local mechanical variations in MGs, the average local shear strain $\eta^{\text {Mises }}$ of $\gamma$-defects (atoms with highest 5\% shear stress) was compared with that of solid-like atoms (atoms with lowest 5\% shear stress). Only 5\% atoms with the highest and lowest von Mises shear stress were chosen to clearly show the mechanical difference between $\gamma$-defects and solid-like entities. One should notice that the characterization of $\gamma$-defects in this section is more strict than previous one.

Figure 3.5 illustrates the comparison between the average shear strain of $\gamma$-defects and the values of solid-like atoms in different compositions. Apparently, $\gamma$-defects exhibit larger shear strain than solid-like atoms in all compositions, suggesting that $\gamma$-defects generally serve as fertile sites to initiate shear events, while the solid-like atoms are mechanically stable and shear resistant. The shear banding localization can therefore be correlated with the fluctuations of atomic shear stress in glasses. In addition, we also modeled the pure shearing for $\mathrm{Ni}_{80} \mathrm{P}_{20} \mathrm{MG}$ (not shown here, since this work is regarding $\mathrm{Cu}-\mathrm{Zr}$ MGs) to detect the instability of $\gamma$-defects in other MGs. The result similarly suggests that $\gamma$-defects exhibit higher mechanical instability than solid-like entities. Moreover, many previous studies reported that the yield strength of $\mathrm{Cu}-\mathrm{Zr}$ MGs also increases with increasing $\mathrm{Cu}$ content (maximum around $\mathrm{Cu}_{65} \mathrm{Zr}_{35}$ composition) [12]. As noted in section 3.3.2, it is found that the average shear stress of $\mathrm{Cu}$ atoms decreases as $\mathrm{Cu}$ fraction increases. Since atomic shear stress is intimately related with local stiffness, such trend may explain the higher overall strength of $\mathrm{Cu}$-rich glasses from another aspect. The possible reason of shear stress reduction (from $\mathrm{Zr}$-rich to $\mathrm{Cu}-$ rich) may be 
attributed to the enrichment of densely packed clusters (pentagonal polyhedra), which is discussed in many studies [120].
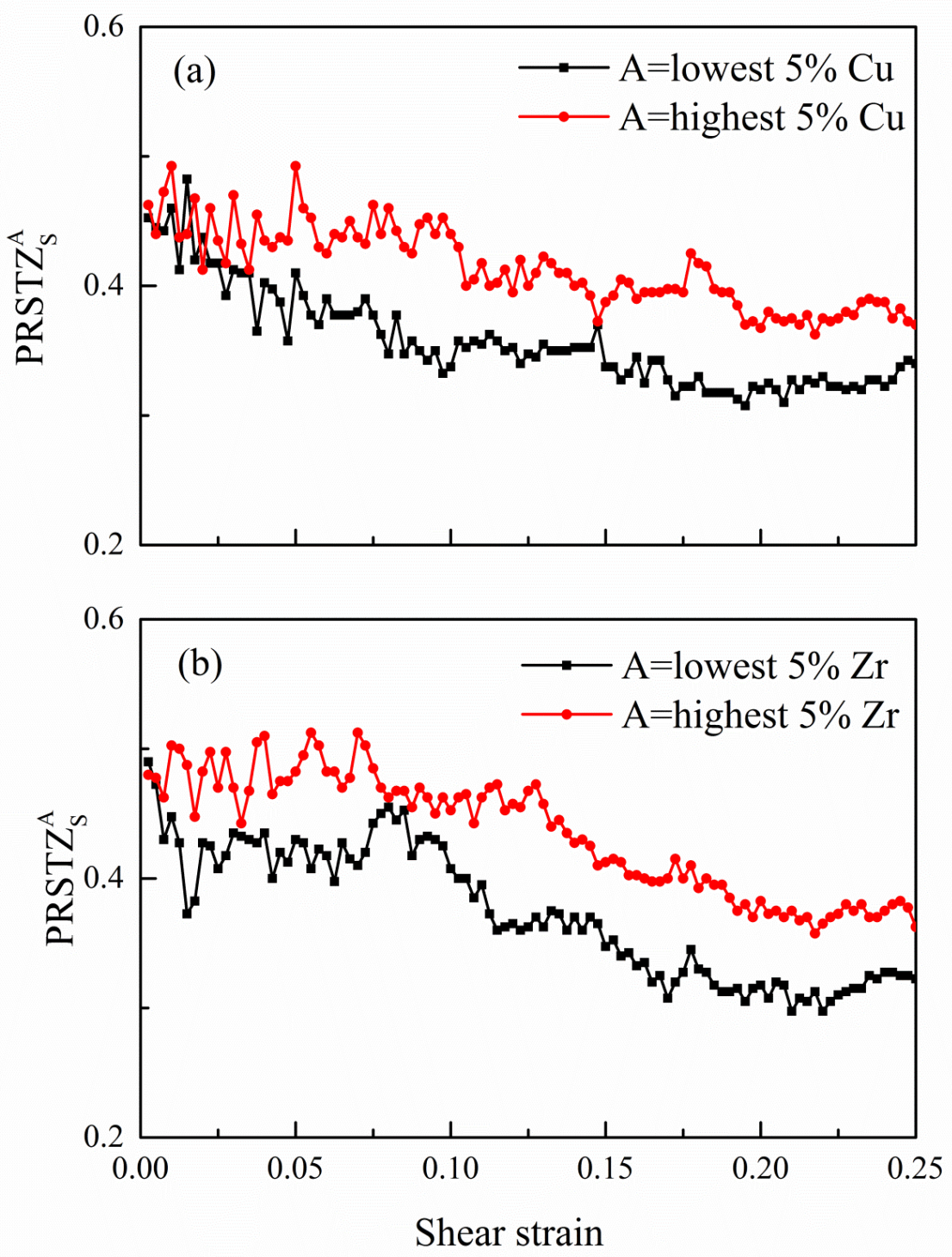

Figure 3.6 The PRSTZ evolutions of $\gamma$-defects and solid-like atoms for (a) $\mathrm{Cu}$ and (b) $\mathrm{Zr}$ atoms during shear deformation in $\mathrm{Cu}_{50} \mathrm{Zr}_{50} \mathrm{MG}$.

As mentioned above, $\gamma$-defects are prone to undergo localized shear events in MGs. To give more information on shear localization, we followed recent studies $[70,132]$ to 
compute the quantitative participation ratio of shear transformation zones ( $\mathrm{PRSTZ}_{S}^{A}$ ) to examine the propensity of defective and stable atoms for strain localization. PRSTZ $\mathrm{P}_{S}^{A}$ represents the fraction of atoms in group $\mathrm{A}$ that possess $\eta_{i}^{\text {Mises }}$ larger than the average shear strain of group $\mathrm{S}$. In this work, group $\mathrm{S}$ represents all $\mathrm{Cu} / \mathrm{Zr}$ atoms, and group $\mathrm{A}$ is respectively defined as $\gamma$-defects and solid-like atoms. If the local shear strain of an atom in group $\mathrm{A}$ is higher than the average shear strain of group $\mathrm{S}$, then this atom is considered to have already participated in plastic deformation, and vice versa. Therefore, $\operatorname{PRSTZ}_{S}^{A}$ can reflect the tendency of atoms in group A to participate in plastic deformation. The $\mathrm{PRSTZ}_{S}^{A}$ of $\gamma$-defects and solid-like atoms in $\mathrm{Cu}_{50} \mathrm{Zr}_{50}$ glass are compared in Figure 3.6. The PRSTZs comparisons of other two compositions are also extracted and exhibit similar features with $\mathrm{Cu}_{50} \mathrm{Zr}_{50}$ glass (not shown here). Clearly, $\gamma$-defects exhibit larger $\operatorname{PRSTZ}_{S}^{A}$ than solid-like atoms, implying that $\gamma$-defects are more preferential to participate in STZs than elastostatic regions.

Rather than Voronoi polyhedra, which only refers to the nearest-neighbor atom arrangements, atomic level stress can provide additional information regarding the medium-range level, since the force field of MD simulations is beyond the first coordination distance. In addition, such concept is generally applicable to identify the structural defects in most glasses and supercooled liquids. Thus this parameter serves as another representative indicator to study the structural fluctuations in MGs. This simulation work proves that local shear properties of MGs are sensitively dependent on the atomic shear stress. Furthermore, it is expected that the atomic level stress may be coupled to the Voronoi polyhedra in some systems, since both low-stress atoms and full icosahedra are shear resistant in $\mathrm{Cu}_{65} \mathrm{Zr}_{35} \mathrm{MG}$ [133]. For instance, Cheng et al. [134] suggested that the shear modulus of $\mathrm{Cu}-\mathrm{Zr}$ MGs is dependent on the atomic level stress, 
implying the intrinsic connections between local fivefold symmetry and atomic shear stress, which may provide further understanding of the structural heterogeneity in MGs. The relationship between atomic level stress and Voronoi index is discussed in the next chapter.

\subsection{Summary}

In conclusion, atomic-level stress fluctuations are closely related with the local kinetic and mechanical responses in modeled $\mathrm{Cu}_{\mathrm{x}} \mathrm{Zr}_{100-\mathrm{x}}$ MGs. Other than Voronoi index, atomic-level stress is proven to be a generic parameter to identify structural defects in MGs. In addition, $\gamma$-defects with high atomic shear stress exhibit faster dynamics than solid-like atoms. The investigations on MSD evolutions suggest that the local dynamics is significantly affected by the von Mises shear stress. Furthermore, by modeling shear deformation processes, it is proposed here that that $\gamma$-defects are also fertile sites for local inelastic deformation. 


\section{CHAPTER 4 SOLID-LIKE AND LIQUID-LIKE NETWORKS IN}

\section{ZR-CU-AL METALLIC GLASS}

Based on MD simulations, this chapter discusses the medium-range superclusters observed in $\mathrm{Zr}_{46} \mathrm{Cu}_{46} \mathrm{Al}_{8}$ MGs. Voronoi tessellation and nearest-neighbor correlation index were adopted to demonstrate the relationships between medium-range networks and local fivefold symmetry. The samples consist of superclusters of atoms with high degree of local fivefold symmetry, and superclusters of atoms with low degree of local fivefold symmetry. In addition, strong avoidance is presented between these two types of atoms. Such spatial heterogeneity is directly linked with the intrinsic mechanical heterogeneity of MGs.

\subsection{Introduction}

MGs are conventionally considered as metastable materials with intrinsic heterogeneity $[12,135,136]$. In recent decades, numerous studies have been carried out to elucidate the structural and mechanical heterogeneity of MGs at microscopic level. One longstanding issue in this field is the characterization of structural order (solid-like) and defects (liquid-like). Typically, liquid-like sites in MGs are expected to exhibit lower stiffness and serve as fertile sites for local shear transformation, which leads to the catastrophic failure at low temperature [137]. In contrast, solid-like regions are relative stable and resistant to external stimuli (thermal perturbations, stress etc). Experimental evidence has been reported regarding the viscoelastic heterogeneity observed in the asdeposited MG film, and the characteristic length $(2.5 \mathrm{~nm})$ seems to be related with the medium-range structure of glasses [138]. 
By means of computer simulations, various structural parameters (e.g., free volume, atomic-level stress, Voronoi clusters) have been proposed to describe the discrepancies between solid-like and liquid-like atoms [48, 66, 139-141]. For instance, the predominance and stability of atoms with high degree of local fivefold symmetry (HLFFS) have been confirmed in many glassy systems [64, 68, 72, 122, 142, 143]. Particularly, icosahedral short-range ordering has aroused extensive attention in the structural motifs of MGs [70, 86, 121, 144]. In some cases, MRO is characterized by several superclusters (or networks) formed by the percolation of icosahedral SRO [145, 146]. Peng et al. [106, 123] and Li et al. [147] conducted MD simulations to explore the spatial correlations and dynamical features of different Voronoi polyhedra in $\mathrm{Cu}-\mathrm{Zr}$ MGs. Their findings include: (i) FI clusters tend to build networks by connecting with one another; (ii) $\mathrm{Cu}$-centered FI also tend to be the nearest neighbors of other HLFFS clusters (such as $\mathrm{Zr}$-centered $\langle 0,1,10,5\rangle$ ), and (iii) HLFFS clusters generally exhibit slower kinetics during annealing process. Cheng et al. [80]investigated the links between local structure and shear properties in $\mathrm{Cu}-\mathrm{Zr}$ and $\mathrm{Cu}-\mathrm{Zr}-\mathrm{Al}$ MGs. The FI backbones and low-population clusters in the glassy samples exhibit different resistance towards shearing. Hwang et al. [148] also proposed the correlations between medium-range structural fluctuations and Voronoi index by implementing RMC simulations. Following previous studies, the MRO of $\mathrm{Cu}-\mathrm{Zr}$ MGs is primarily attributed to the interconnection of FI, and such backbones exhibit high stability under external disturbances. On the other hand, low-population polyhedra are assumed to be unfavorable or liquid-like entities in glasses [149]. Nevertheless, the characteristics of those low-population clusters remain a long-standing mystery for researchers, hampering our understanding of the medium-range structure and structure-property relationship in MGs. 
Another indicator to identify the defective sites in MGs is the atomic level stress theory proposed by Egami et al. [66]. This concept attributes the topological instability of structural defects to the excessive stresses on atoms. Based on Egami's criterion, atoms with higher stress and strain energy are considered to be unfavorable and liquid-like. Cheng et al. [118] carried out atomistic simulations to examine the intrinsic links between the local topology and atomic strain energy in $\mathrm{Cu}_{64} \mathrm{Zr}_{36}$ and $\mathrm{Pd}_{82} \mathrm{Si}_{18}$ MGs. Particularly, the bifurcation of $\mathrm{CN}$ and atomic volume is attributed to the fluctuations of atomic level pressure. As aforementioned in chapter 3, atomic level stress is also linked with the local dynamic and mechanical properties of atoms in $\mathrm{Cu}-\mathrm{Zr}$ MGs. Atomic level stress seems to be a good indicator to characterize the structural order and defects on the short-range scale. To a certain extent, Voronoi index and atomic level stress theory provide similar explanation of the solid-like and liquid-like atoms in glasses. Therefore it would be interesting and necessary to examine the relationships between atomic-level stress and Voronoi index in MGs.

In this chapter, MD simulations were implemented to get an insight into the atomic landscape of $\mathrm{Zr}_{46} \mathrm{Cu}_{46} \mathrm{Al}_{8} \mathrm{MG}$, which is a typical model used to study the characteristics of BMGs. Such structural model can reveal the spatial arrangements of atoms (structural heterogeneity) on the medium-range scale, although there exist inevitable discrepancies between simulated and real-world MGs. The medium-range structure in MGs is directly linked with the local fivefold symmetry (Voronoi index). Solid and liquid-like networks in the MGs are thus defined according to such correlations. Additionally, the mechanical differences between solid-like and liquid-like regions are studied by modeling a shear deformation process. Furthermore, this chapter correlates the structural inhomogeneity to the atomic level stress distribution in glasses. Such correlations may give valuable 
explanation of microscopic mechanisms underlying the structural and mechanical heterogeneity in MGs.

\subsection{Simulation methods}

\subsubsection{Computational models}

LAMMPS [128, 150] software was adopted to investigate the characteristics of solid and liquid-like networks in glassy metals. Classical MD simulation was adopted to obtain a 54,000-atom configuration of $\mathrm{Zr}_{46} \mathrm{Cu}_{46} \mathrm{Al}_{8} \mathrm{MG}$, with interatomic interactions determined by EAM potentials. PBCs were applied on three axial directions. The initial positions of $\mathrm{Zr}, \mathrm{Cu}$ and $\mathrm{Al}$ atoms were randomly arranged in a bcc supercell. The system initial temperature was kept at $2000 \mathrm{~K}$ for 2,3 and 4 ns respectively. NPT quenching (zero pressure) from the melts $(2000 \mathrm{~K})$ to $50 \mathrm{~K}$ at a cooling rate of $10^{11} \mathrm{~K} / \mathrm{s}$ was then performed to obtain the amorphous samples. Averaged results of these $3 \mathrm{MG}$ models were investigated to explore the solid and liquid-like regions in $\mathrm{Zr}_{46} \mathrm{Cu}_{46} \mathrm{Al}_{8} \mathrm{MG}$. In addition, von Mises shear strain $\eta_{i}^{\text {Mises }}$ was monitored to unveil the intrinsic relationships between solid-like/liquid-like sites and local shear resistance, by employing the open visualization tool (OVITO) software [151]. To detect the structure-property correlations, the sample obtained at the cooling rate of $10^{11} \mathrm{~K} / \mathrm{s}$ was deformed along the $x z$ direction by using supercell tilting at $50 \mathrm{~K}$. Moreover, the average atomic shear stress on various polyhedral types was computed to demonstrate the suitability of atomic-level stress theory regarding the short and medium-range structure of MGs.

\subsubsection{Calculation of nearest-neighbor correlation index}

On the short-range scale, the microstructures of MGs are representatively demonstrated by the Voronoi tessellation method [64]. Following previous simulation studies [106], the spatial distributions of various Voronoi clusters on the medium-range scale can be 
revealed by monitoring the nearest-neighbor correlation index $C_{i j}$ between central atoms of polyhedral types $i$ and $j$. According to Ref. [147], the number of the nearest neighbors of types $i$ and $j$ is denoted as $m_{i j}$, and $P_{\text {total }}$ represents the total number of nearest-neighbor pairs in the whole system. Then we denote the probability of polyhedral types $i$ and $j$ being nearest neighbors as $p_{i j}=m_{i j} / P_{\text {total }}$. On the other hand, if Vornoi clusters are spatially uncorrelated (randomly distributed), then the probability of polyhedral types $i$ and $j$ being nearest neighbors is merely determined by their fractions, and this parameter is given by

$$
p_{i j}^{0}=\left\{\begin{array}{l}
\frac{2 n_{i} n_{j}}{N(N-1)}(i \neq j) \\
\frac{n_{i}\left(n_{j}-1\right)}{N(N-1)}(i=j)
\end{array}\right.
$$

where $n_{i}\left(n_{j}\right)$ represents the number of atoms of index $i(j)$, and $N$ is the total number of atoms in the system. Then the nearest-neighbor correlation index is determined by

$$
C_{i j}=\frac{p_{i j}}{p_{i j}^{0}}-1
$$

Accordingly, $C_{i j}$ represents the spatial correlations between the central atoms of different polyhedra types. A random distribution of polyhedra types $i$ and $j$ gives a zero value of $C_{i j}$. Positive values of $C_{i j}$ indicate strong correlations (or connectivity) between polyhedra $i$ and $j$ (the central atoms of polyhedra types $i$ and $j$ are likely to be nearest neighbors), while negative values indicate anticorrelation (there is strong avoidance between the central atoms of polyhedra types $i$ and $j$ ). 


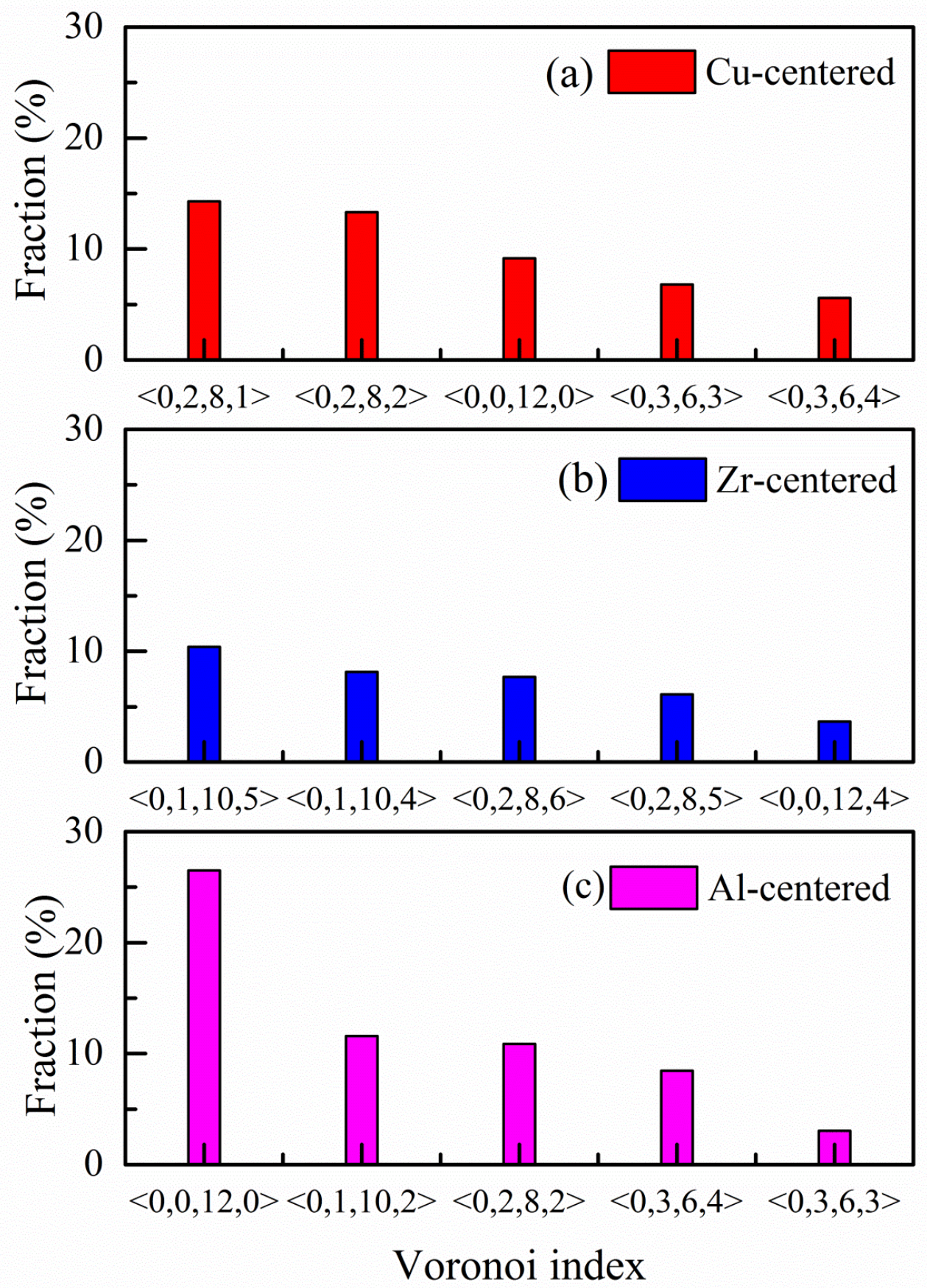

Figure 4.1 Fractions of the most populated Voronoi polyhedra in $\mathrm{Zr}_{46} \mathrm{Cu}_{46} \mathrm{Al}_{8} \mathrm{MG}$. 


\subsection{Results and discussion}

\subsubsection{Solid-like and liquid-like networks on the medium-range scale}

To examine the microstructures of the simulated glasses, atomic coordinates are firstly analyzed by employing radical Voronoi tessellation method. The Voronoi index $\left\langle n_{3}, n_{4}, n_{5}, n_{6}\right\rangle$ is extracted to describe the topological arrangements of atoms on the short-range scale. Figure 4.1 shows the fractions of the dominant coordination polyhedra in $\mathrm{Zr}_{46} \mathrm{Cu}_{46} \mathrm{Al}_{8} \mathrm{MG}$ (obtained at a cooling rate of $10^{11} \mathrm{~K} / \mathrm{s}$ ). The results present the prevalence of HLFFS atoms in the glass, such as $\mathrm{Cu}$ or Al-centered $<0,0,12,0>\mathrm{FI}$ and Zr-centered $\langle 0,1,10,5\rangle$ etc. Such finding shows good agreement with other simulation studies [71]. In general, HLFFS atoms are believed to exhibit higher stability and stiffness, and their interconnections give rise to the MRO or solid-like backbones in MGs $[152,153]$. On the other hand, there is still a pressing demand to interpret the structural and mechanical features of low-population clusters, such as atoms with low degree of local fivefold symmetry (LLFFS).

By computing the nearst-neighbor correlation index $C_{i j}$, Figure 4.2 illustrates the spatial correlations of various Voronoi polyhedral. Clusters with different number of pentagonal faces (from high to low) are systematically investigated in the diagram, in order to explain the dissimilar structural features of HLFFS and LLFFS atoms. The correlation strengths for different clusters can be represented by the color schemes in such matrix. For instance, a strong avoidance between two polyhedral types is marked by a dark blue element, while a preference for interpenetrating networks is colored in red. Additionally, the correlation map is separated into nine regions demarcated by the thick black lines, representing the correlations of different pairs $(\mathrm{Cu}-\mathrm{Cu}, \mathrm{Cu}-\mathrm{Zr}, \mathrm{Cu}-\mathrm{Al}, \mathrm{Zr}-\mathrm{Zr}, \mathrm{Zr}-\mathrm{Al}$ and $\mathrm{Al}-\mathrm{Al}$ ). Since the ratio of $\mathrm{Al}$ element is quite low in the $\mathrm{Zr}_{46} \mathrm{Cu}_{46} \mathrm{Al}_{8}$ system, the correlation map of Al-Al pair mainly illustrates the chemical affinity of Al-Al bonds. 


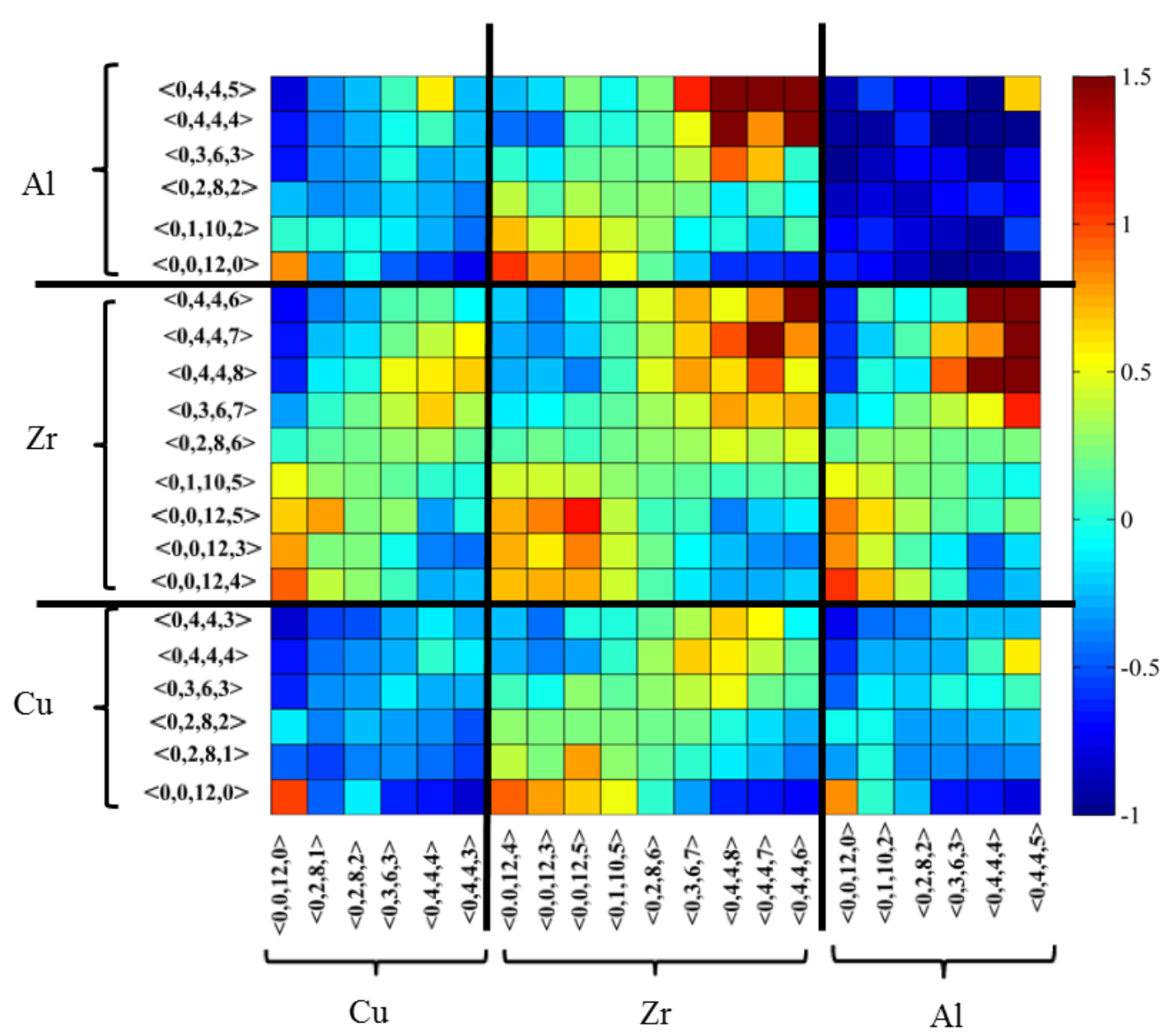

Figure 4.2 Correlation matrix of different coordination polyhedra in $\mathrm{Zr}_{46} \mathrm{Cu}_{46} \mathrm{Al}_{8} \mathrm{MG}$.

The colors represent the correlation strength (see text for details).

As presented in the figure, connections between $\mathrm{Al}$ and $\mathrm{Al}$ atoms are rarely found in the system, indicating the strong avoidance of Al-Al pair. In addition, the correlation strengths between two types of Al-centered clusters may be misleading and hence are neglected in this work. An exception is the correlation index between Al-centered <0, 0 , 12, 0$\rangle$ and Al-centered $\langle 0,0,12,0\rangle$, as the Al-centered FI has relatively large populations in the samples. Although numerous studies suggested the preference of FI to form interpenetrated networks, it is observed in the figure that Al-centered FI tends to keep away from one another. Such observation is validated by the RDFs from 
experimental and MD results, as the first peak of partial Al-Al RDF disappears in $\mathrm{Zr}-\mathrm{Cu}-$ Al MGs [71].

For the other five pairs, the lower left and upper right corners generally exhibit positive values of $C_{i j}$, while the lower right and upper left corners exhibit negative values of $C_{i j}$. Such findings suggest that HLFFS atoms likely to build up percolated networks (MRO), which is typically believed to be the stable backbones in MGs. In addition, similar networks (or superclusters) formed by the interconnection of LLFFS atoms are also observed in this work, which have rarely reported in previous experiments and simulations. On the other hand, strong avoidance is observed between HLFFS and LLFFS atoms, indicating the significant discrepancies between these two types of atoms, which may implicit the structural and mechanical heterogeneity in MGs. In other words, LLFFS atoms are not isolated but aggregated into networks or superclusters. The correlation length of such networks is several nanometers, showing good agreement with the characteristic length of mechanical heterogeneity reported in MGs (1 to $10 \mathrm{~nm}$ ) [138, 154-158]. For instance, $\mathrm{Cu}$-centered $\langle 0,0,12,0\rangle$ are likely to percolate with $\mathrm{Cu}-$ centered $\langle 0,0,12,0\rangle$, Al-centered $\langle 0,0,12,0\rangle, \mathrm{Zr}$-centered $\langle 0,0,12,3\rangle,\langle 0,0,12,4\rangle$ and $\langle 0,0,12,5\rangle$. However, $\mathrm{Cu}$-centered $\langle 0,0,12,0\rangle$ shows strong avoidance with LLFFS atoms, such as $\mathrm{Cu}$ or $\mathrm{Al}$-centered $\langle 0,4,4,4\rangle$ and $\mathrm{Zr}$-centered $\langle 0,4,4,8\rangle$. Moreover, $\mathrm{Cu}$-centered $<0,4,4,4>$ tends to connect with other LLFFS atoms. 21 polyhedral types are selected in this work to investigate the spatial correlations of different polyhedra. In addition to these 21 polyhedral types, the correlation indices between other HLFFS and LLFFS atoms (not shown here) also present similar features. Owing to the distinct discrepancies between HLFFS and LLFFS atoms, and the similar length scales of the medium-range networks and the mechanical heterogeneity, HLFFS and LLFFS networks are expected to be solid-like and liquid-like regions. Interestingly, 
our findings are remarkably consistent with a recent published work by $\mathrm{Hu}$ et al. [159]. Figure 4.3 denotes the correlations between the local fivefold symmetry and the spatial distributions of atoms in $\mathrm{Cu}_{46} \mathrm{Zr}_{46} \mathrm{Al}_{8}$ and $\mathrm{Mg}_{65} \mathrm{Cu}_{25} \mathrm{Y}_{10}$ glass-forming liquids. At the medium-range level, the degree of local fivefold symmetry also significantly determines the spatial distributions of different Voronoi clusters.
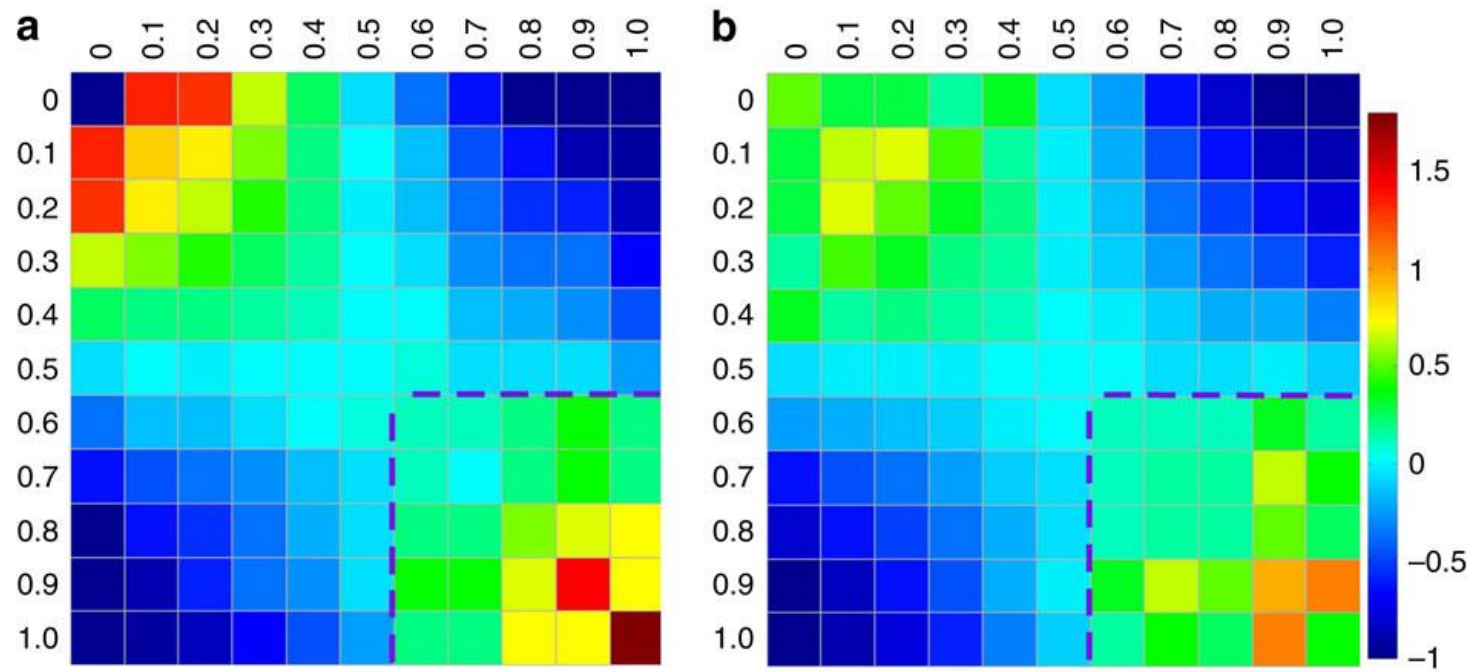

Figure 4.3 Correlation matrix of atoms with difference degree of local fivefold symmetry in (a) $\mathrm{Cu}_{46} \mathrm{Zr}_{46} \mathrm{Al}_{8}$ and (b) $\mathrm{Mg}_{65} \mathrm{Cu}_{25} \mathrm{Y}_{10}$ glass-forming liquids at $T=1.2 T_{g}$ [159]. (The correlation maps exhibit similar features at different temperatures).

The correlation matrix suggests the inhomogeneous distribution of HLFFS and LLFFS atoms in the $\mathrm{Zr}_{46} \mathrm{Cu}_{46} \mathrm{Al}_{8}$ MG. To obtain a straightforward insight into the structural heterogeneity, the distribution and configurations of HLFFS and LLFFS networks (or superclusters) are shown in Figures 4.4(a) and (b). There is not a clear criterion about how many pentagonal faces should correspond to a HLFFS or LLFFS polyhedron. However for simplicity of explanation, in the following we characterize atoms with twelve pentagonal faces as HLFFS particles (such as $\mathrm{Cu}$ and $\mathrm{Al}$-centered $\langle 0,0,12,0\rangle$, Zr-centered $\langle 0,0,12,3\rangle,\langle 0,0,12,3\rangle,\langle 0,0,12,4\rangle$ ), while atoms with no more than four pentagonal faces as LLFFS particles (such as Al-centered $\langle 0,4,4,5\rangle, \mathrm{Cu}$-centered 
$<1,3,4,4\rangle$, Zr-centered $\langle 0,4,4,7\rangle)$. There is not a clear criterion about how many pentagonal faces should correspond to a HLFFS or LLFFS polyhedron. However for simplicity of explanation, in the following we characterize atoms with twelve pentagonal faces as HLFFS atoms (such as $\mathrm{Cu}$ and Al-centered $\langle 0,0,12,0\rangle, \mathrm{Zr}$-centered $\langle 0,0,12,3\rangle,\langle 0,0,12,3\rangle,\langle 0,0,12,4\rangle)$, while atoms with no more than four pentagonal faces as LLFFS atoms (such as Al-centered $\langle 0,4,4,5\rangle, \mathrm{Cu}$-centered $<1,3,4,4\rangle$, Zr-centered $\langle 0,4,4,7\rangle$ ). Other atoms with 5 to 11 pentagonal faces are hence referred to as intermediate states.

The networked HLFFS atoms (green spheres) are clearly depicted in Figure 4.4(a), representing the MRO or solid-like backbones in MGs. LLFFS atoms (red spheres) also display spatial heterogeneity and build interpenetrated networks. Nevertheless, the correlations between HLFFS and LLFFS atoms are found to be relatively weak. Figure 4.4(b) displays the configurations of one HLFFS and one LLFFS supercluster, indicating the medium-range structures in this system. Accordingly, the HLFFS and LLFFS networks provide revealing information regarding the structural heterogeneity on the medium-range level, and these networks may fundamentally determine the dynamical and mechanical heterogeneity in MGs. Specifically, the LLFFS superclusters may serve as defective sites in relation with the overall properties of MGs.

Although the medium-range structures in MGs are intimately linked with the local fivefold symmetry (Voronoi indices), here we stress that chemical effects also play an important role in determining the medium-range networks. For instance, although FI are likely to build percolated networks, the anticorrelation between Al-centered FI themselves is observed and mainly caused by the strong chemical avoidance of Al-Al pair. However, such relationship is beyond the scope of this work. 
(a)

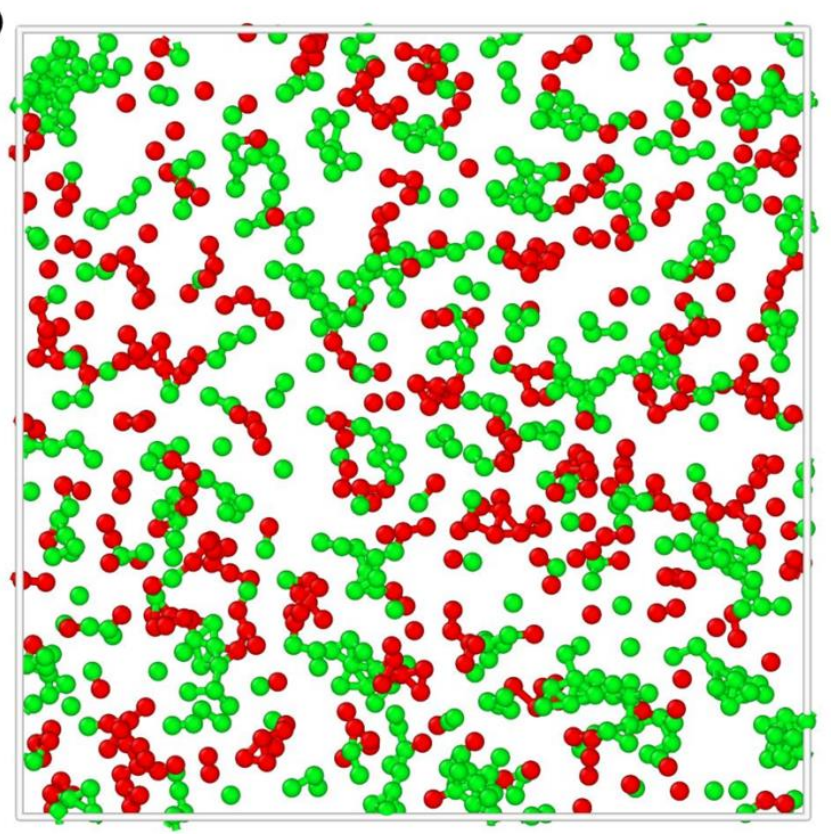

(b)
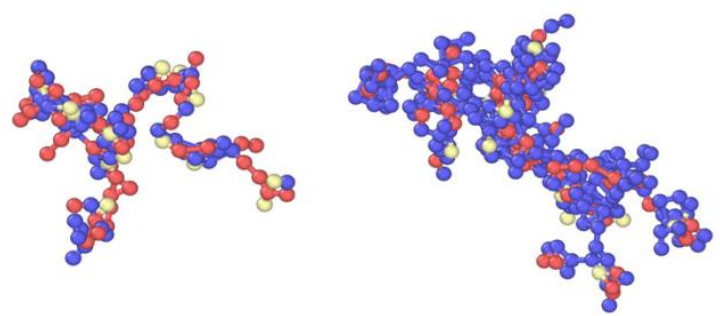

Figure 4.4 (a) Spatial distributions and connections of HLFFS and LLFFS atoms in the $\mathrm{Zr}_{46} \mathrm{Cu}_{46} \mathrm{Al}_{8} \mathrm{MG}$ within a thickness of $6 \AA$. Only HLFFS and LLFFS atoms are displayed for clarity. For the spatial heterogeneity in glass, HLFFS atoms (colored green) are percolated and form networks, and LLFFS atoms (colored red) have the similar tendency. The connectivity between HLFFS and LLFFS atoms is weak. (b) 3D configurations of two networks respectively formed by HLFFS and LLFFS atoms. The $\mathrm{Cu}, \mathrm{Zr}$ and $\mathrm{Al}$ atoms are colored purple, red and yellow, respectively. 


\subsubsection{Influences of atomic level stress on local topology}

The structural heterogeneity of MGs can be correlated with the atomic level stress and its distribution. Previous studies reported the influence of atomic level pressure on atomic sizes, CNs and short-range order $[66,118]$. In our study, the degree of fivefold symmetry in MGs is observed to be related with atomic shear stress. Additionally, the stress fluctuations are found to extend on the medium-range scale, indicating that the short-to-medium range order is significantly influenced by the local stress (energy) of atoms.

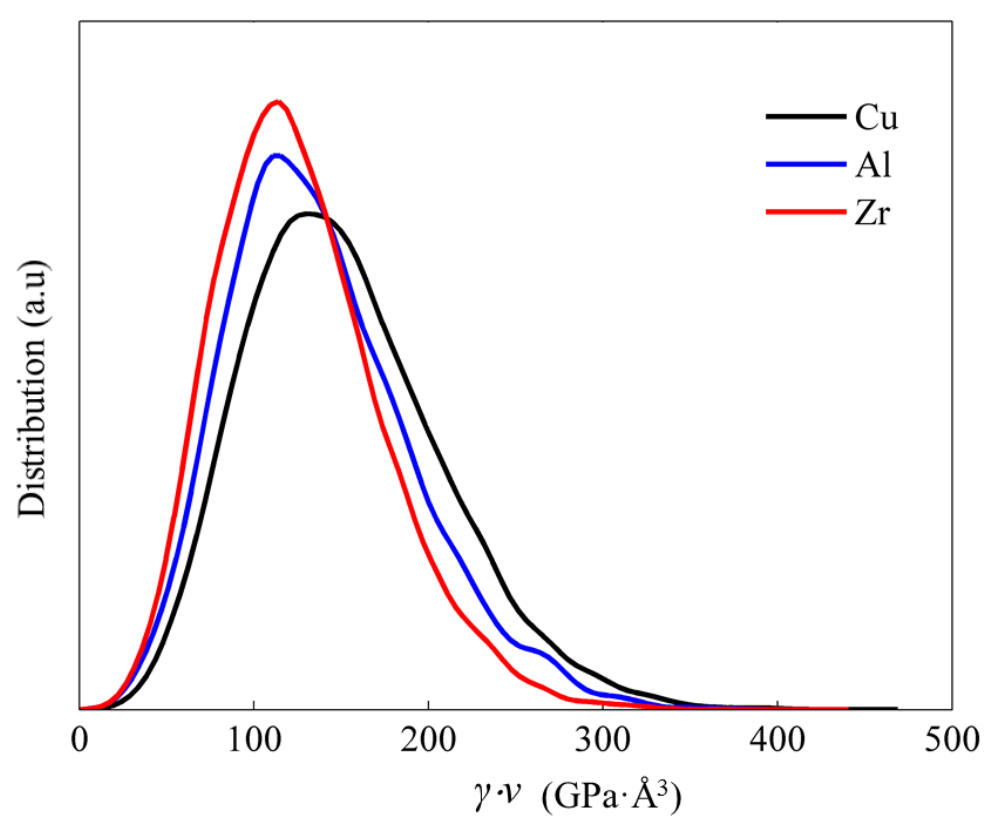

Figure 4.5 Distribution of atomic shear stress multiplied by atomic volume in $\mathrm{Zr}_{46} \mathrm{Cu}_{46} \mathrm{Al}_{8}$ MG. $\sigma_{i} \cdot v_{i}$ could be directly extracted from the MD simulation; to further calculate the atomic level shear stress, the atomic volume for each atom should be defined (or use the average atomic volume).

The atomic level stress for each atom can be derived following the original work of Ref.. This work will focus on the shear component, that is, the von Mises shear stress. Figure 4.5 illustrates the atomic shear stress distribution of three different elements in our 
model, which is non-Gaussian like. According to Egami et al. [66], atoms with excessive shear stress are likely to be plastically deformed and are thus defined as $\gamma$-defects. Interestingly, we found a close correlation between atomic shear stress and coordination types by calculating the average shear stress on different polyhedra, as shown in Table 4.1. It is observed that HLFFS atoms (like FI) typically have smaller shear stress, while LLFFS atoms possess larger shear stress. This suggests that the local topology in MGs is strongly related to the atomic level stress on each atom. However, atomic level stress is only an average parameter and unable to clearly reflect local structural and chemical distortions. A quantitative theory is still required to explain the structural inhomogeneity in MGs.

Table 4.1 The average atomic shear stress (multiplied by atomic volume) for different Voronoi polyhedra in $\mathrm{Zr}_{46} \mathrm{Cu}_{46} \mathrm{Al}_{8} \mathrm{MG}$.

\begin{tabular}{cccccc}
\hline \hline Cu-centered & $\begin{array}{c}\text { Atomic } \\
\text { shear stress } \\
\left(\mathrm{GPa} \cdot \AA^{3}\right)\end{array}$ & Zr-centered & $\begin{array}{c}\text { Atomic } \\
\text { shear stress } \\
\left(\mathrm{GPa} \cdot \AA^{3}\right)\end{array}$ & Al-centered & $\begin{array}{c}\text { Atomic } \\
\text { shear stress } \\
\left(\mathrm{GPa} \cdot \AA^{3}\right)\end{array}$ \\
\hline$<0,0,12,0>$ & 113.83 & $<0,0,12,4>$ & 146.91 & $<0,0,12,0>$ & 128.21 \\
$<0,2,8,2>$ & 129.06 & $<0,1,10,5>$ & 148.13 & $<0,2,8,2>$ & 145.18 \\
$<0,3,6,3>$ & 127.03 & $<0,2,8,6>$ & 150.00 & $<0,3,6,3>$ & 142.92 \\
$<0,4,4,4>$ & 128.26 & $<0,3,6,7>$ & 152.49 & $<0,4,4,4>$ & 150.98 \\
& $<0,4,4,8>$ & 163.92 & & \\
\hline \hline
\end{tabular}

In addition, to study the relationship between atomic shear stress and spatial distribution of atoms, we sorted atoms of the same species according to the magnitude of the atomic shear stress (from low to high). All the atoms sorted are binned into five groups, with each group containing $20 \%$ of the total atoms and having larger atomic shear stress than the previous group. Specifically, we computed the correlation index $C_{i j}$ for those atoms with highest or lowest shear stress, as shown in Table 4.2. The total average values of $C_{i j}$ indicate the overall spatial correlations for different pairs, and the values represent the 
chemical affinity of different pairs. Specifically, we found that the highest-highest and lowest-lowest pairs possess larger $C_{i j}$ than the total average value, indicating that atoms with similar shear stress are likely to agglomerate together. The smaller $C_{i j}$ of highestlowest pair suggests the avoidance between high-stress and low-stress atoms. It is therefore concluded that atoms with similar shear stress are likely to be interconnected, forming the medium-range networks in MGs.

Table 4.2 Spatial correlation index $C_{i j}$ of atoms with highest/lowest atomic shear stress.

\begin{tabular}{ccccc}
\hline \hline & \multicolumn{4}{c}{ Spatial correlation index $\left(C_{i j}\right)$} \\
\cline { 2 - 5 } & Total average & $\begin{array}{c}\text { lowest-lowest } \\
\text { pair }\end{array}$ & $\begin{array}{c}\text { lowest-highest } \\
\text { pair }\end{array}$ & $\begin{array}{c}\text { highest-highest } \\
\text { pair }\end{array}$ \\
\hline $\mathrm{Cu}-\mathrm{Cu}$ & -0.38 & -0.21 & -0.41 & -0.41 \\
$\mathrm{Zr}-\mathrm{Zr}$ & 0.20 & 0.23 & 0.14 & 0.30 \\
$\mathrm{Cu}-\mathrm{Zr}$ & 0.11 & 0.18 & 0.02 & 0.18 \\
$\mathrm{Cu}-\mathrm{Al}$ & -0.23 & -0.19 & -0.24 & -0.16 \\
$\mathrm{Zr}-\mathrm{Al}$ & 0.18 & 0.18 & 0.08 & 0.40 \\
$\mathrm{Al}-\mathrm{Al}$ & -0.74 & -0.68 & -0.80 & -0.72 \\
\hline \hline
\end{tabular}

Furthermore, the spatial heterogeneity in MGs is influenced by each component of the stress tensor. Figures 4.6(a) and (b) respectively illustrate how the spatial distributions of atoms are affected by $\sigma_{i}^{x x}$ and $\tau_{i}^{x y}$ in the system (the influence of $\sigma_{i}^{y y}$ and $\sigma_{i}^{z z}$ should be similar to $\sigma_{i}^{x x}$ and that of $\tau_{i}^{x z}$ and $\tau_{i}^{y z}$ should be similar to $\tau_{i}^{x y}$ ). Only atoms with excessive positive stresses (colored red) and atoms with excessive negative values (colored blue) are displayed in these two figures for clarity. Our results reveal that atoms with similar $\sigma_{i}^{x x}$ or $\tau_{i}^{x y}$ tend to percolate among themselves, and the connections are found to reach the medium-range scale $(\mathrm{nm})$. Accordingly, all the stress components (three normal stresses and three shear stresses) should contribute to the spatial heterogeneity founded in MGs, involving the short-to-medium range structure of the simulated glass. Based on above investigations, we can therefore conclude that the short- 
to-medium range structure mainly reflect the stress distribution (which also implies the local energetic stability) in MGs.
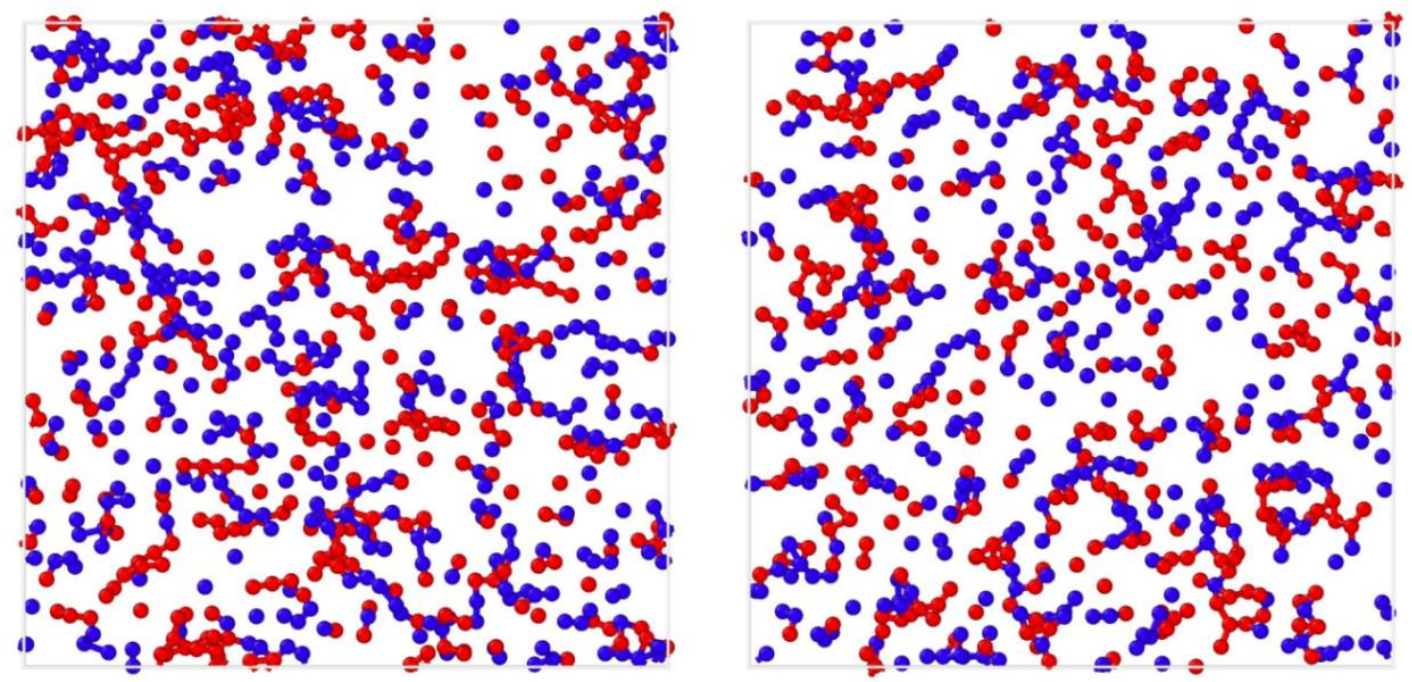

Figure 4.6 Spatial distribution of $\mathrm{Cu}$ atoms with excessive positive/negative atomic stress in terms of (a) $\sigma_{i}^{x x}$ and (b) $\tau_{i}^{x y}$ within a thickness of $6 \AA$, to illustrate the dependence of atomic level stress on spatial heterogeneity in MGs. Only atoms with excessive stress are displayed for clarity. Atoms with excessive positive stress (colored red) are heterogeneously distributed and tend to form percolated networks, and atoms with excessive negative stress (colored blue) have the similar trend, indicating atoms with similar stress are likely to be percolated.

During the rapid cooling process of MGs, naturally some atoms can find relatively "comfortable" environment (corresponding to low-stress states), while some are "unhappy" and unstable (corresponding to high-stress states). Those low-stress (stable) particles cluster together and form the solid-like matrix, whereas high-stress (unstable) particles similarly build up liquid-like superclusters. This study reveals the existence of the medium-range networks formed by LLFFS atoms, as well as the mechanisms 
underlying the structural heterogeneity in MGs. Thus, local stress or energy fluctuations account for the structural heterogeneity found in amorphous systems.

\subsubsection{Mechanical discrepancies between solid-like and liquid-like regions}

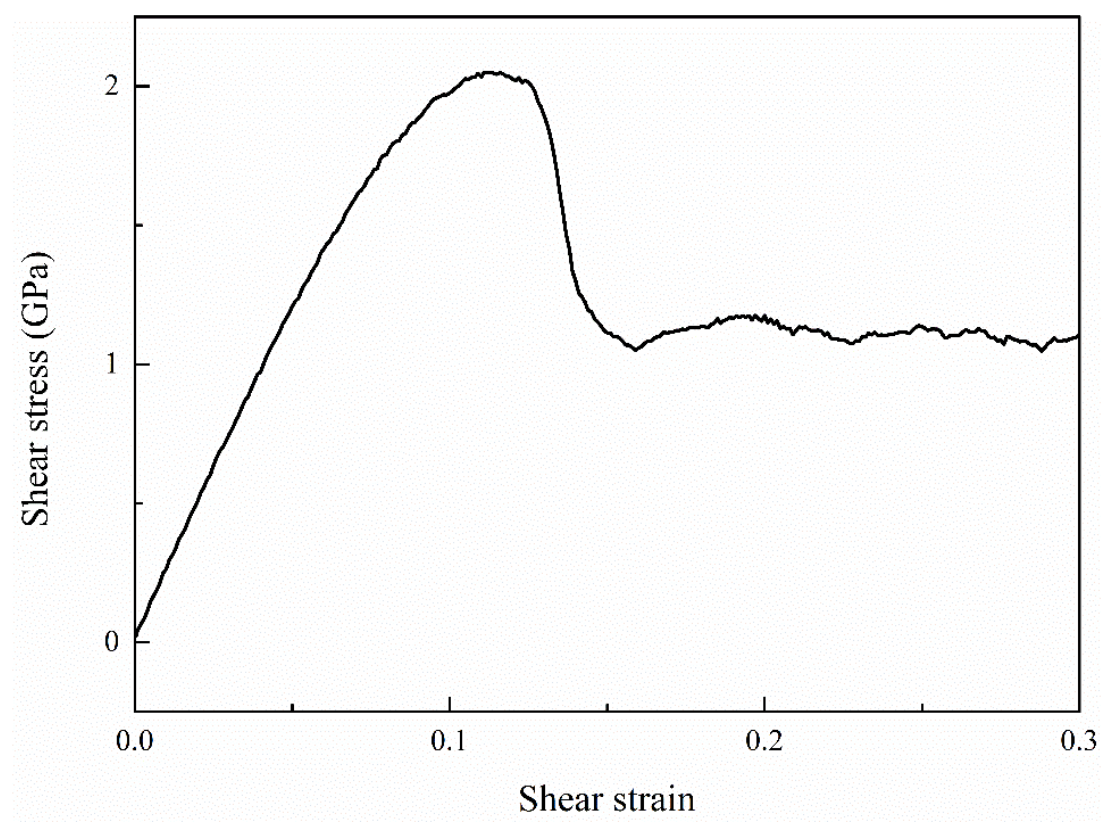

Figure 4.7 Overall stress-strain curve of $\mathrm{Zr}_{46} \mathrm{Cu}_{46} \mathrm{Al}_{8} \mathrm{MG}$ under pure shear loading.

Figure 4.7 shows the overall shear stress-strain curve of $\mathrm{Zr}_{46} \mathrm{Cu}_{46} \mathrm{Al}_{8} \mathrm{MG}$. In the plastic regime $(\varepsilon>7 \%)$, liquid-like atoms commence to be plastically deformed, because structural defects are expected to exhibit instabilities under shear deformation. With increasing stress, more and more liquid-like atoms are activated to connect with each other, leading to the irreversible macroscopic strain. As we mentioned in previous sections, the networks formed by HLFFS and LLFFS atoms are predicted as solid and liquid-like regions respectively. To validate this assumption, their mechanical performances during shear deformation are examined by tracking the von Mises shear strain $\eta_{i}^{\text {Mises }}$ of each atom. Similarly, atoms with twelve pentagonal faces are defined as HLFFS particles, while atoms with no more than four pentagonal faces are LLFFS particles. Figure 4.7 shows the average shear strain $\eta^{\text {Mises }}$ for different groups of atoms. 
At a strain larger than 7\%, the HLFFS atoms exhibit smaller $\eta^{\text {Mises }}$ than that for all atoms, whereas LLFFS atoms exhibit larger $\eta^{\text {Mises }}$ than the average value. This finding suggests the mechanical instability of LLFFS regions, and their high propensity for plastic deformation under external loads. Additionally, the HLFFS networks are relatively stable and exhibit high resistance to shear transformations. 

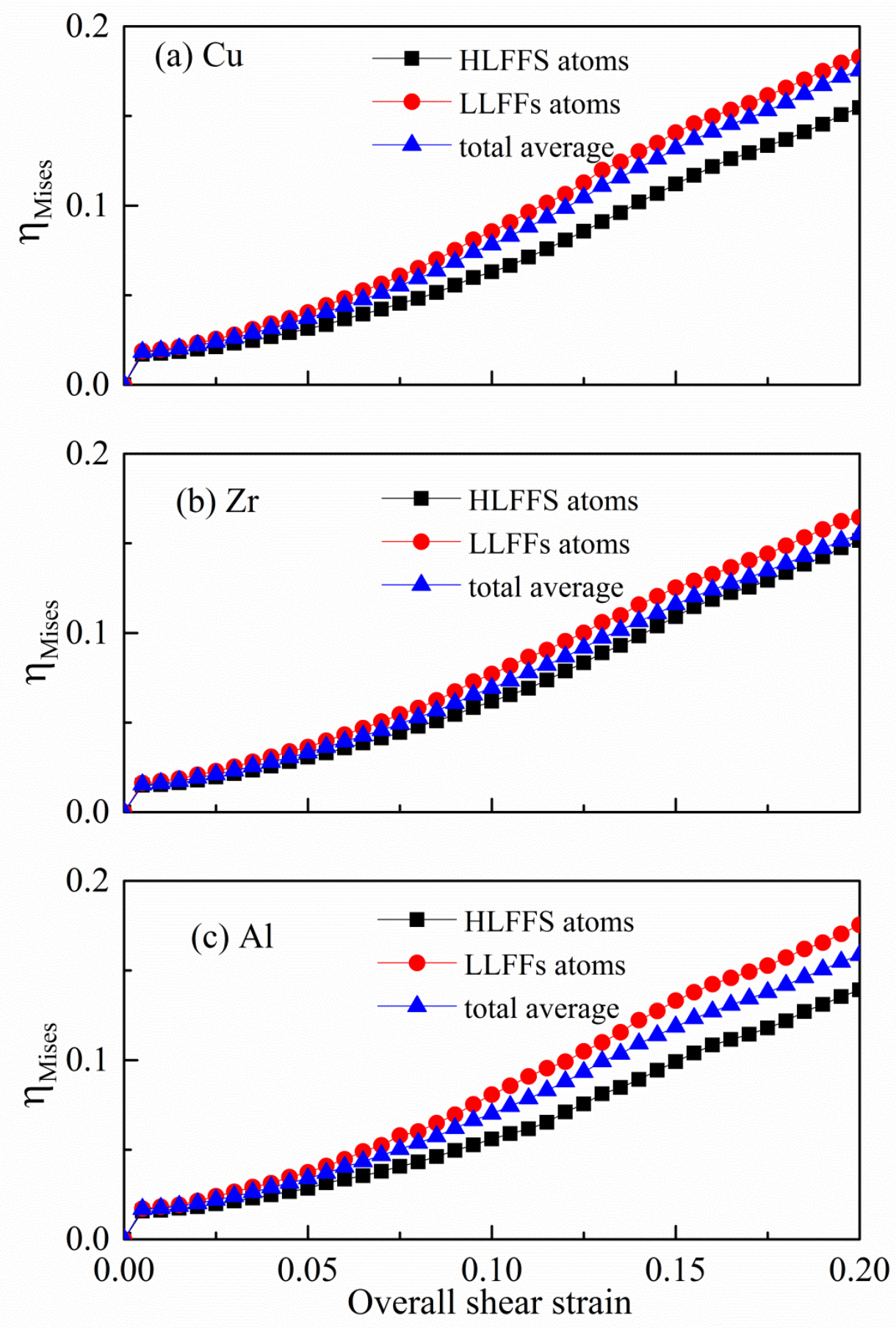

Figure 4.8 The average atomic shear strain evolution of HLFFS and LLFFS atoms for (a) $\mathrm{Cu}$, (b) $\mathrm{Zr}$ and (c) Al versus overall shear strain during the pure shear deformation of $\mathrm{Zr}_{46} \mathrm{Cu}_{46} \mathrm{Al}_{8} \mathrm{MG}$. 


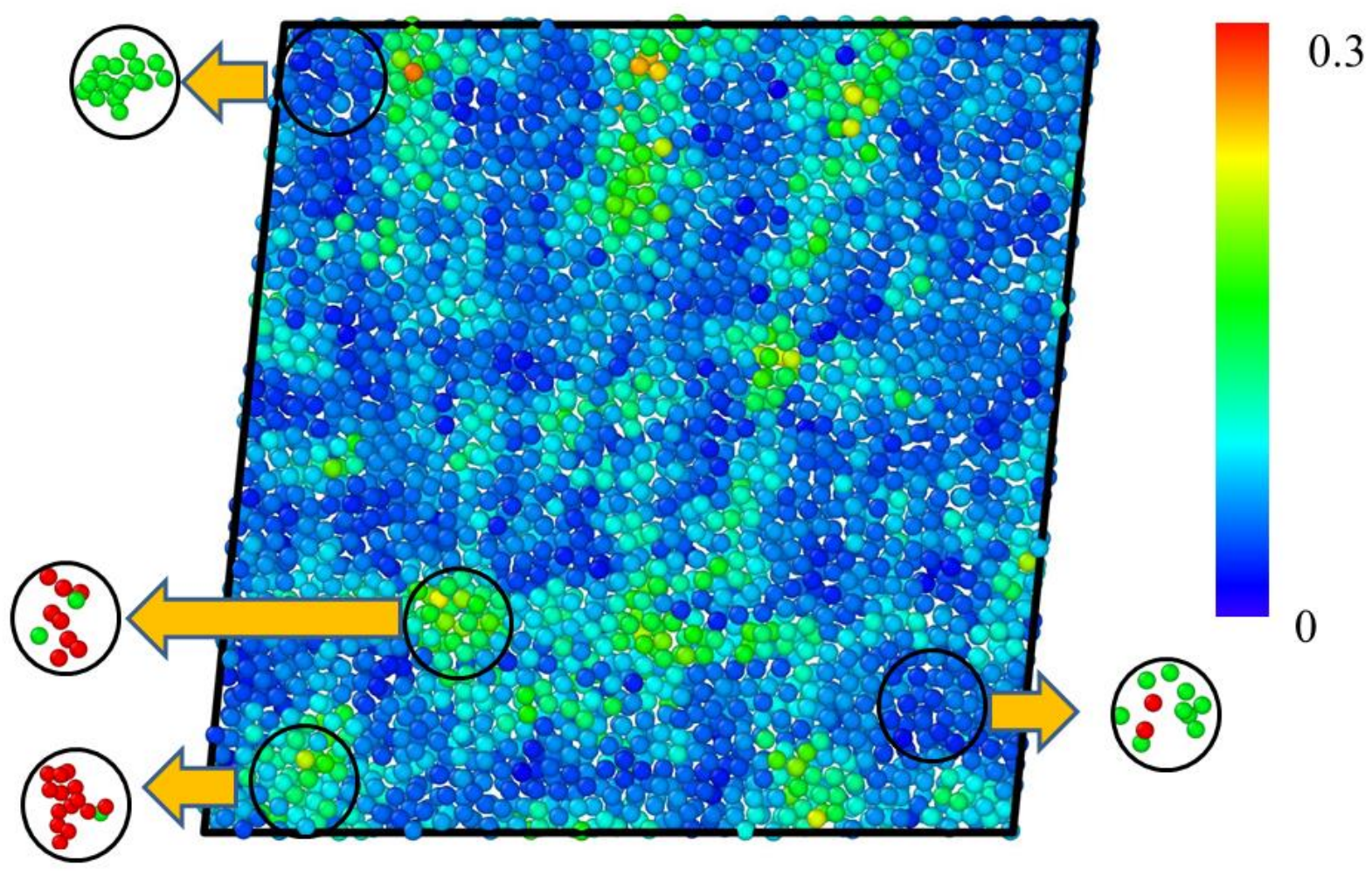

Figure 4.9 Correlations between local plastic deformations and HLFFS and LLFFS atoms. This figure displays the cross-section view of a deformed $\mathrm{Zr}_{46} \mathrm{Cu}_{46} \mathrm{Al}_{8}$ sample, at shear strain of $10 \%$. The colors are mapped to the various levels of von Mises shear strains. The dark blue regions are elastically deformed while the green ones have participated in plastic deformations. Two elastic and two plastic regions are circled out and their local structures are illustrated in the insets. For clarity, only HLFFS and LLFFS atoms are shown in the insets. For the elastic regions, HLFFS atoms (colored green) are dominant and few LLFFS atoms (colored red) are observed. While in the plastically deformed regions, LLFFS atoms are abundant and HLFFS atoms are rarely found. 
To study how HLFFS networks resist local plastic deformations and how LLFFS networks serve as fertile sites for shear transformations, a specific configuration of our deformed model is shown in Figure 4.8. Regions with high fraction of HLFFS atoms are elastically deformed (indicated by small shear strain), while regions with high fraction of LLFFS atoms are likely to participate in shear transformations (indicated by large shear strain). Therefore, our results suggest that the HLFFS and LLFFS networks should be the solid- and liquid-like regions in this model, especially in terms of the mechanical heterogeneity of MGs. The plastic flow is initiated mainly by the flow of liquid-like networks formed by LLFFS atoms, which may account for the shear transformation zones (STZs) and shear localization phenomenon in MGs.

\subsection{Summary}

In summary, we monitored the networks formed by HLFFS and LLFFS atoms in $\mathrm{Zr}_{46} \mathrm{Cu}_{46} \mathrm{Al}_{8} \mathrm{MG}$. Our analysis provides an insight into the structural and mechanical heterogeneity of MGs. In addition to the solid-like backbones formed by HLFFS atoms, there are also networks formed by LLFFS atoms, which represent the liquid-like (unstable) regions in glassy metals. Our quantitative analysis in terms of atomic level stress reveals the correlations between atomic shear stress and local fivefold symmetry (regarding the short-range structure in MGs). Additionally, the spatial connections of atoms (medium-range structure) are significantly affected by the atomic level stress. These findings provide the microscopic origins of structural heterogeneity in MGs. The mechanical heterogeneity of MGs was investigated by simulating a pure shear loading process. It is revealed that LLFFS atoms are likely to initiate plastic flow, while HLFFS entities are resistant to plastic deformation. Such observation validates our assumption that the HLFFS and LLFFS networks respectively represent the solid-like and liquid-like regions in this model. 
CHAPTER 5 CHEMICAL SHORT RANGE ORDER AND ITS

CORRELATIONS

WITH

DYNAMIC/MECHANICAL

\section{HETEROGENEITIES IN ZR-CU-AG METALLIC GLASS}

This chapter examines the formation of chemical short range order and its influences on the dynamic and mechanical heterogeneities in $\mathrm{Zr}_{45} \mathrm{Cu}_{45} \mathrm{Ag}_{10}$ alloys. MG samples were prepared under different cooling histories via classical MD simulations. The temperature dependence of Warren-Cowley parameter reveals the rapid growth of chemical short range order during the supercooling process. In addition, mean square displacement and von Mises shear strain were extracted to respectively investigate the dynamic and mechanical heterogeneities in these samples.

\subsection{Introduction}

The unique attributes of MGs, e.g. high yielding strength, good corrosion resistance, low-temperature brittleness, are commonly believed to stem from the topological and chemical fluctuations at the microscopic level $[12,160]$. Over the past decades, the TSRO and its effects on the macroscopic and microscopic characteristics of MGs have been extensively explored by experiments and simulations. As aforementioned, various theories and parameters, such as free volume, atomic-level stress, $\mathrm{CN}$, Voronoi index, have been proposed to unveil the characteristics of TSRO in MGs $[49,66,72,80,118$, 161]. For instance, the high-frequency dynamic micropillar tests by Ye et al. [156] revealed that the free volume zones account for the local inelastic deformation in MGs. Li et al. [162] also claimed the similar conclusions by implementing atomistic simulations. In addition, numerous simulations suggested the prevalence and stability of 
local fivefold symmetry in MGs. An experimental observation of local atomic order has also been achieved by conducting nanobeam electron diffraction tests [163].

On the other hand, there are only a few studies devoted to monitor how chemical makeup affects the local properties in MGs. By using atomistic simulations, solute-centered clusters were reported as the representative motifs in various glass-forming systems [64]. The results suggested the existence of CSRO in amorphous alloys, which is termed as the "solute-solute avoidance" phenomena. In some cases, CSRO parameters were extracted to examine the temperature and composition dependence of CSRO in MGs [164-166]. In addition, the relaxation dynamics of $\mathrm{Cu}-\mathrm{Zr}-\mathrm{Ag}$ MGs has been examined by Fujita et al. [167]. The displacement measurements confirmed that atoms in Ag-rich regions exhibit faster dynamics than those in Ag-poor regions, which may explain the improved glass forming ability (GFA) with $\mathrm{Ag}$ addition to $\mathrm{Cu}-\mathrm{Zr}$ alloys. Moreover, plasticity enhancement has been discovered in many phase-separated multicomponent MGs, which typically has positive enthalpy of mixing between two alloying elements [160]. Chen et al. [168], Sung et al. [169] and Park et al. [170] reported the plastic limit up to $16.3 \%, 8 \%$ and $4.2 \%$ in as-quenched $\mathrm{Cu}_{47.2} \mathrm{Zr}_{46.5} \mathrm{Al}_{5.5} \mathrm{Nb}_{0.8}, \mathrm{Cu}_{43} \mathrm{Zr}_{43} \mathrm{Al}_{7} \mathrm{Ag}_{7}$ and $\mathrm{Cu}_{47} \mathrm{Ti}_{33} \mathrm{Zr}_{7} \mathrm{Nb}_{4} \mathrm{Ni}_{8} \mathrm{Si}_{1}$ BMGs, respectively. In $\mathrm{Cu}_{43} \mathrm{Zr}_{43} \mathrm{Al}_{7} \mathrm{Ag}_{7}$ glass, the $\mathrm{Cu}$-enriched and Ag-enriched zones are experimentally detected and expected to exhibit different mechanical properties [171]. Accordingly, chemical inhomogeneity may be regarded as a possible reason for the ductility improvement in those phase-separated alloys. Therefore, an atomistic simulation study is necessary for understanding the microscopic characteristics of the intrinsic chemical fluctuations in multicomponent MGs.

As phase separation has been observed in $\mathrm{Ag}-\mathrm{Cu}-\mathrm{Zr}$ systems [172], this work aims to figure out the underlying relationships between chemical, dynamic and mechanical inhomogeneity in $\mathrm{Zr}_{45} \mathrm{Cu}_{45} \mathrm{Ag}_{10}$ glasses. By using classical MD simulations, amorphous 
$\mathrm{Zr}_{45} \mathrm{Cu}_{45} \mathrm{Ag}_{10}$ alloys were prepared under different cooling rates. On the basis of these simulations, CSRO parameters, atomic potential energy, MSDs and von Mises shear strain were extracted. The results demonstrate that the formation of CSRO is significantly attributed to the improved energetic stability during supercooling. Additionally, the local dynamic and mechanical properties of MGs are strongly dependent on the chemical heterogeneities.

\subsection{Simulation methods}

\subsubsection{Computational models}

An open code software for classic MD simulations, LAMMPS, was adopted to study the CSRO and mechanical performances of MGs [128]. Our simulation model was $\mathrm{Zr}_{45} \mathrm{Cu}_{45} \mathrm{Ag}_{10}$ made of 16,000 atoms. The EAM potential was employed to determine the interatomic interactions in the systems. The initial positions of $\mathrm{Zr}, \mathrm{Cu}$ and $\mathrm{Ag}$ atoms were randomly allocated in a bcc supercell according to their proportions. Periodic boundary conditions (PBCs) were employed along $x, y$ and $z$ directions to avoid edge effects. The MD timestep was set as 1 fs to eliminate discretization errors. To obtain a fully melted system, the model was firstly equilibrated at $2000 \mathrm{~K}$ for $2 \mathrm{~ns}$. To obtain $\mathrm{Zr}_{45} \mathrm{Cu}_{45} \mathrm{Ag}_{10} \mathrm{MGs}$, isothermal isobaric (NPT) cooling from the melts to $50 \mathrm{~K}$ was then conducted under different quench rates of $10^{10}, 10^{11}$ and $10^{12} \mathrm{~K} / \mathrm{s}$. After quenching, the systems were kept at $50 \mathrm{~K}$ for another $2 \mathrm{~ns}$ for equilibration. The dynamic properties of the models were analyzed by simulating annealing processes (20 runs) at constant temperature $(800 \mathrm{~K})$. The configurations and atom coordinates were collected and averaged over the simulation duration (100 ps) for structural and dynamic analyses. By performing a pure shear loading along $x z$ direction, the shear properties of the samples were examined. The strain rate was set as $10^{8} \mathrm{~s}^{-1}$. The temperature was kept at $50 \mathrm{~K}$ during the shear deformation, in order to highlight the material responses under 
mechanical activations rather than thermal perturbations. As mentioned previously, RDF, CN, MSD and von Mises shear strain were extracted to study the characteristics of chemical fluctuations in these samples.

\subsubsection{Chemical short range order parameter}

The CSRO parameter can be calculated by analyzing the chemical make-up of the nearest neighbor atoms, and comparing it with the composition of the alloy. This reflects the deviation between the local chemistry and the expectation of a random solution. The Warren-Cowley parameter is conventionally adopted to quantify the CSROand is defined as [173]

$$
\alpha_{i j}=1-\frac{Z_{i j}}{\chi_{j} Z_{i}}
$$

where $Z_{i j}$ is the partial coordination number $(\mathrm{CN})$ of $j$ atoms around $i$ atoms, $Z_{i}$ represents the total $\mathrm{CN}$ around $i$, and $\chi_{j}$ is the fraction of $j$. Accordingly, a negative value of $\alpha_{i j}$ indicates that the chemical bonds are favored, while a positive value means that the bonds are unfavorable. Finally, $\alpha_{i j}=0$ represents random solution.

\subsection{Results and discussion}

\subsubsection{Glass formation process}

Figure 5.1 shows the glass formation of a simulated $\mathrm{Zr}_{45} \mathrm{Cu}_{45} \mathrm{Ag}_{10} \mathrm{MG}$, quenched under zero pressure and cooling rates of $10^{11} \mathrm{~K} / \mathrm{s}$. Typically, the glass transitions of MG can be characterized by the presence of continuity in volume $(V)$, potential energy $(E)$ or enthalpy $(H)$ change as a function of temperature $(T)$. During the cooling process, the glass transition takes place around the glass transition temperature $\left(T_{g}\right)$. The figure illustrates that the glass transition temperature for $\mathrm{Zr}_{45} \mathrm{Cu}_{45} \mathrm{Ag}_{10} \mathrm{MG}$ is around $690 \mathrm{~K}$, 
which exhibits remarkable consistency with the experimental data of $683 \mathrm{~K}$ reported by Zhang et al. [174]. The 3D configuration of the simulated glass is also shown in the inset of Figure 5.1.

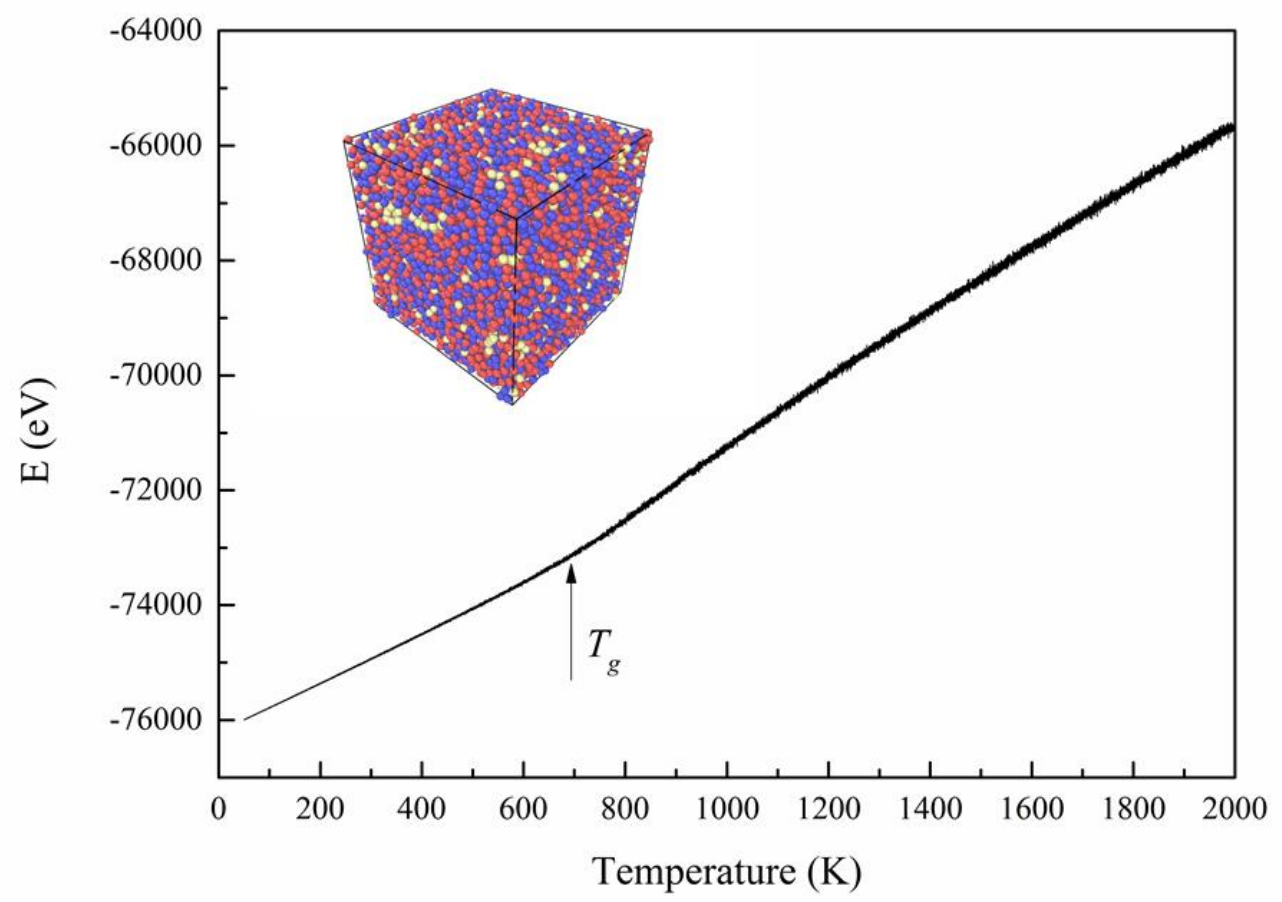

Figure 5.1 Temperature dependence of potential energy in $\mathrm{Zr}_{45} \mathrm{Cu}_{45} \mathrm{Ag}_{10}$ alloy during cooling from $2000 \mathrm{~K}$ to $50 \mathrm{~K}$. 3D configuration of the $\mathrm{Zr}_{45} \mathrm{Cu}_{45} \mathrm{Ag}_{10} \mathrm{MG}$ sample is shown in the inset. $\mathrm{Zr}, \mathrm{Cu}$ and $\mathrm{Ag}$ atoms are colored red, purple and yellow respectively. 


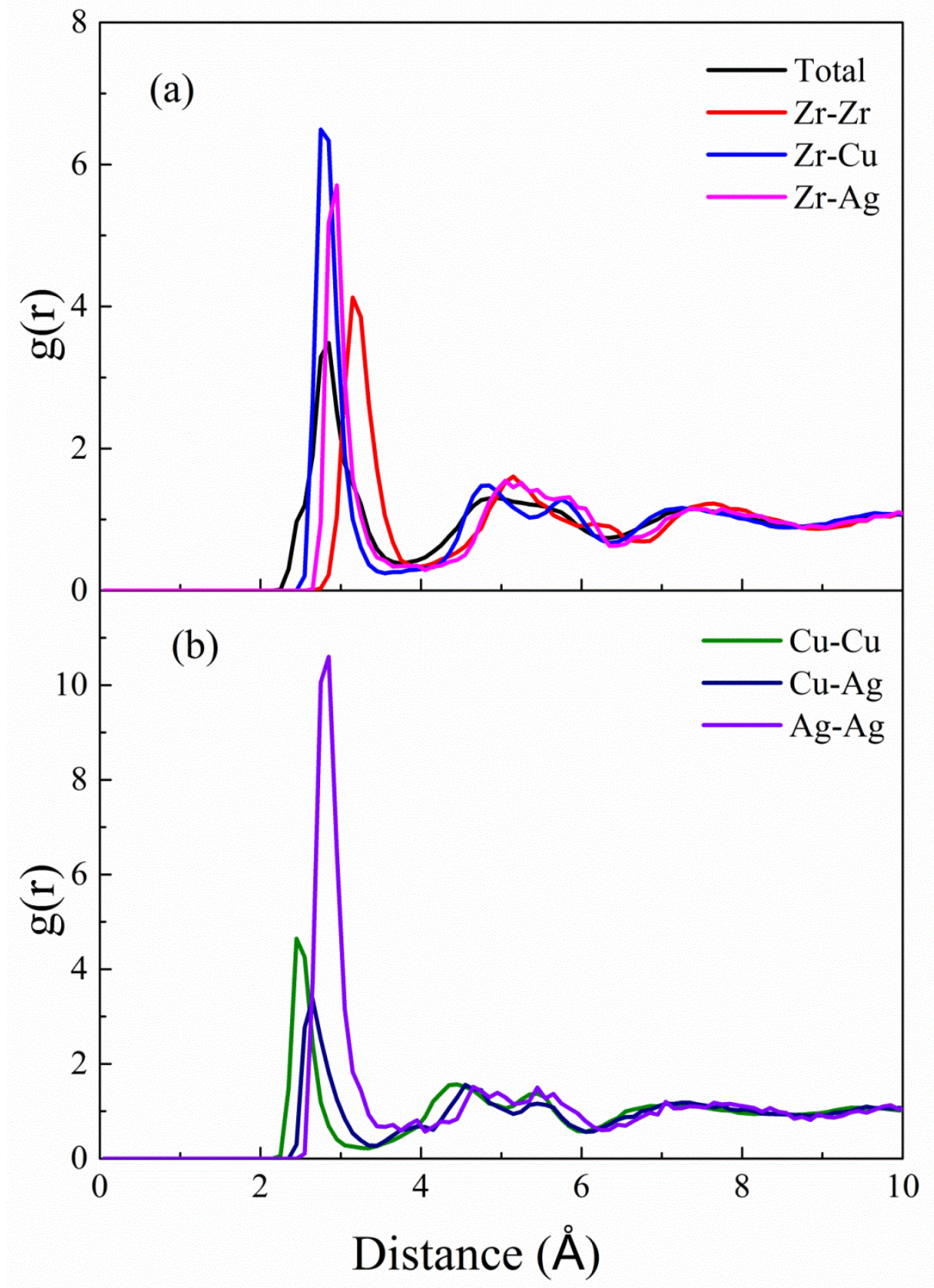

Figure 5.2 Total and partial RDFs of $\mathrm{Zr}_{45} \mathrm{Cu}_{45} \mathrm{Ag}_{10} \mathrm{MG}$.

Figures 5.2 (a) and (b) show the total and partial RDFs for the amorphous sample at 50 $\mathrm{K}$. Although the Ag content is quite low, we observe a pronounced RDF of Ag-Ag pair from such figure. This observation indicates the strong affinity for Ag-Ag pairs in the $\mathrm{Zr}-\mathrm{Cu}-\mathrm{Ag} \mathrm{MGs}$, which shows agreement with previous studies [167]. Moreover, the 
PRDFs were generated to extract the average interatomic distances of different pairs, which were listed in Table 5.1. The general agreement of these values with the Goldschmidt radii $\left(R_{Z r}=1.60 \AA, R_{C u}=1.28 \AA, R_{A g}=1.44 \AA\right)$ reveals the metallic nature of the ternary MGs. Following previous studies [12], the first minima of the PRDFs were chosen to determine the first coordination shell and obtain the partial and total $\mathrm{CNs}$. Based on the figure, the cutoff values of $\mathrm{Zr}-\mathrm{Zr}, \mathrm{Zr}-\mathrm{Cu}, \mathrm{Zr}-\mathrm{Ag}, \mathrm{Cu}-\mathrm{Cu}, \mathrm{Cu}-\mathrm{Ag}$ and Ag-Ag pairs are $4.15 \AA, 3.75 \AA, 3.95 \AA, 3.45 \AA, 3.45 \AA$ and $3.55 \AA$, respectively. The average partial and total CNs for different species are listed in Table 5.2.

Table 5.1 The interatomic distances $(\AA)$ for different pairs in $\mathrm{Zr}_{45} \mathrm{Cu}_{45} \mathrm{Ag}_{10} \mathrm{MG}$.

\begin{tabular}{ccccccc}
\hline \hline $\begin{array}{c}\text { Interatomic } \\
\text { distances }(\AA)\end{array}$ & $R_{\mathrm{Zr}-\mathrm{Zr}}$ & $R_{\mathrm{Zr}-\mathrm{Cu}}$ & $R_{\mathrm{Zr}-\mathrm{Ag}}$ & $R_{\mathrm{Cu}-\mathrm{Cu}}$ & $R_{\mathrm{Cu}-\mathrm{Ag}}$ & $R_{\mathrm{Ag}-\mathrm{Ag}}$ \\
\hline MD & 3.16 & 2.84 & 2.96 & 2.46 & 2.65 & 2.85 \\
Goldschmidt & 3.20 & 2.88 & 3.04 & 2.48 & 2.72 & 2.88 \\
\hline \hline
\end{tabular}

Table 5.2 Partial and total coordination number for different elements in $\mathrm{Zr}_{45} \mathrm{Cu}_{45} \mathrm{Ag}_{10}$ MG.

\begin{tabular}{cccccc}
\hline \hline $\mathrm{Z}_{\mathrm{Zr}-\mathrm{Cu}}$ & 6.99 & $\mathrm{Z}_{\mathrm{Cu}-\mathrm{Cu}}$ & 4.19 & $\mathrm{Z}_{\mathrm{Ag}-\mathrm{Cu}}$ & 4.47 \\
$\mathrm{Z}_{\mathrm{Zr}-\mathrm{Zr}}$ & 7.30 & $\mathrm{Z}_{\mathrm{Cu}-\mathrm{Zr}}$ & 6.99 & $\mathrm{Z}_{\mathrm{Ag}-\mathrm{Zr}}$ & 6.57 \\
$\mathrm{Z}_{\mathrm{Zr}-\mathrm{Ag}}$ & 1.46 & $\mathrm{Z}_{\mathrm{Cu}-\mathrm{Ag}}$ & 0.99 & $\mathrm{Z}_{\mathrm{Ag}-\mathrm{Ag}}$ & 2.72 \\
$\mathrm{Z}_{\mathrm{Zr}}$ & 15.74 & $\mathrm{Z}_{\mathrm{Cu}}$ & 12.17 & $\mathrm{Z}_{\mathrm{Ag}}$ & 13.76 \\
\hline \hline
\end{tabular}

\subsubsection{Formation of chemical short range order}

Naturally, there exist significant discrepancies between real-world glasses and their ideal states. Such frustration gives rise to the stable (solid-like) and defective (liquid-like) zones reported in MGs, which can be linked with their topological and chemical heterogeneity. Usually, the topological heterogeneity can be monitored by examining some topology parameters, such as free volume, atomic level stress and Voronoi index. 
On the other hand, knowledge on the CSRO of MGs has not been well established, despite recent investigation by Fujita and colleagues in that direction [167]. Therefore in this work, we will focus on the chemical inhomogeneity and its effects in the amorphous $\mathrm{Zr}-\mathrm{Cu}-\mathrm{Ag}$ alloys.

Table 5.3 Comparison of Warren-Cowley parameters at $2000 \mathrm{~K}$ and $50 \mathrm{~K}$ in $\mathrm{Zr}_{45} \mathrm{Cu}_{45} \mathrm{Ag}_{10} \mathrm{MGs}$ obtained at different cooling rates.

\begin{tabular}{lcccc}
\hline \hline & $2000 \mathrm{~K}$ & \multicolumn{3}{c}{$50 \mathrm{~K}$} \\
\cline { 3 - 5 } & & $10^{10} \mathrm{~K} / \mathrm{s}$ & $10^{11} \mathrm{~K} / \mathrm{s}$ & $10^{12} \mathrm{~K} / \mathrm{s}$ \\
\hline$\alpha_{\mathrm{Ag}-\mathrm{Ag}}$ & -0.287 & -1.225 & -0.994 & -0.713 \\
$\alpha_{\mathrm{Ag}-\mathrm{Cu}}$ & 0.103 & 0.314 & 0.273 & 0.234 \\
$\alpha_{\mathrm{Ag}-\mathrm{Zr}}$ & -0.039 & -0.042 & -0.052 & -0.075 \\
$\alpha_{\mathrm{Cu}-\mathrm{Ag}}$ & -0.006 & 0.210 & 0.164 & 0.122 \\
$\alpha_{\mathrm{Cu}-\mathrm{Cu}}$ & 0.155 & 0.249 & 0.255 & 0.254 \\
$\alpha_{\mathrm{Cu}-\mathrm{Zr}}$ & -0.154 & -0.296 & -0.291 & -0.281 \\
$\alpha_{\mathrm{Zr}-\mathrm{Ag}}$ & 0.046 & 0.088 & 0.081 & 0.060 \\
$\alpha_{\mathrm{Zr}-\mathrm{Cu}}$ & 0.055 & 0.015 & 0.019 & 0.023 \\
$\alpha_{\mathrm{Zr}-\mathrm{Zr}}$ & -0.065 & -0.034 & -0.037 & -0.036 \\
\hline \hline
\end{tabular}

The Warren-Cowley parameters for different pairs were tracked to quantify the formation of CSRO during the supercooling of the sample (take the sample obtained at $10^{12} \mathrm{~K} / \mathrm{s}$ for example), as listed in Table 5.3. The chemical inhomogeneity typically exists in both liquid and glass states. For instance, the CSRO values of Ag-Ag pairs at $2000 \mathrm{~K}$ deviates a lot from zero (-0.287), indicating that the chemical preference of such pair can still be observed in liquid states. Then the chemical inhomogeneity grows rapidly as the model is cooled. Comparing the Warren-Cowley parameters at $2000 \mathrm{~K}$ with the values at $50 \mathrm{~K}$, one can readily figure out the chemical preference of different bonds in the amorphous alloys. Interestingly, the CSRO parameters for $\mathrm{Zr}-\mathrm{Zr}$, $\mathrm{Zr}-\mathrm{Ag}$ and $\mathrm{Zr}-\mathrm{Cu}$ remain almost unchanged (close to zero) before and after cooling. This finding indicates that the neighboring environments have little impacts on the stability of central $\mathrm{Zr}$ atoms, which is in good agreement with the experimental observations by $\mathrm{Oh}$ et al. 
[171]. Therefore in this work, we will focus on the CSRO distribution of $\mathrm{Cu}$ and $\mathrm{Ag}$ atoms. For central $\mathrm{Cu}$ and $\mathrm{Ag}$ atoms, it can be observed that the CSRO parameters of $\mathrm{Cu}-\mathrm{Zr}$ and $\mathrm{Ag}-\mathrm{Ag}$ pairs become smaller (negative) as the alloy is quenched. The CSRO values of $\mathrm{Ag}-\mathrm{Cu}, \mathrm{Cu}-\mathrm{Ag}$ and $\mathrm{Cu}-\mathrm{Cu}$ become larger (positive), whereas the $\mathrm{Ag}-\mathrm{Zr}$ pair remains similar (close to zero). These findings suggest that the $\mathrm{Cu}-\mathrm{Zr}$ and $\mathrm{Ag}-\mathrm{Ag}$ bonds tend to chemically stabilize the central $\mathrm{Cu}$ and $\mathrm{Ag}$ atoms, respectively. On the other hand, the $\mathrm{Cu}-\mathrm{Ag}$ and $\mathrm{Cu}-\mathrm{Cu}$ bonds hamper the local stability of central $\mathrm{Cu}$ atoms. The $\mathrm{Ag}-\mathrm{Cu}$ interactions also play a similar role on central Ag atoms. Furthermore, the Ag- $\mathrm{Zr}$ bonds correspond to the intermediate states for central Ag atoms. The CSRO evolution during the cooling process is illustrated in Figure 5.3. As seen in the figure, the CSRO becomes prominent in the supercooled regime, which is similar to the development of TSRO [73]. According to the figure, the chemical preference and avoidance become prominent throughout the supercooled liquid region, leading to the heterogeneous distribution of atoms in space. Similar to the topological heterogeneity, the chemical heterogeneity may result in the variation of local dynamic and mechanical properties, which is discussed in the following sections.

Moreover, the degree of CSRO is found to be highly affected by the cooling history of MGs. With decreasing cooling rates, one can see that the favored pairs become more prominent, while those unstable bonds tend to be suppressed. For instance, the WarrenCowley parameter of $\mathrm{Ag}-\mathrm{Ag}$ pairs decreases with reduced quench rates, implicating the remarkable growth of stable $\mathrm{Ag}-\mathrm{Ag}$ bonds. In contrast, the $\mathrm{CSRO}$ value of $\mathrm{Ag}-\mathrm{Cu}$ pairs rises with decreasing cooling rates, demonstrating the growing avoidance between central $\mathrm{Ag}$ and neighboring $\mathrm{Cu}$ atoms. Such tendency is also comparable to the TSRO formation, as previous simulations reported the change of icosahedral SRO with different cooling histories [71]. 


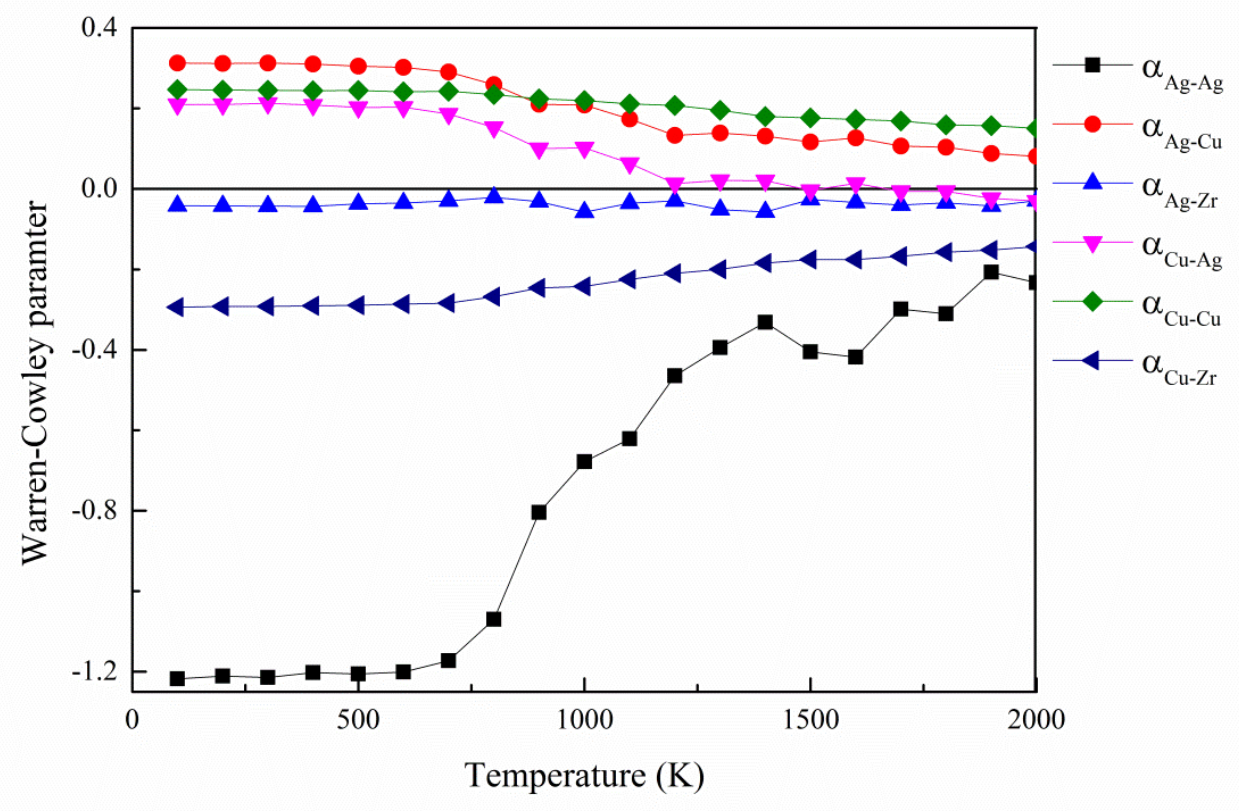

Figure 5.3 Temperature dependence of Warren-Cowley parameters for different pairs during the cooling process of $\mathrm{Zr}_{45} \mathrm{Cu}_{45} \mathrm{Ag}_{10}$ alloy. $\mathrm{Zr}-(\mathrm{Cu} / \mathrm{Zr} / \mathrm{Ag})$ pairs are omitted for clarity.

Table 5.4 Average potential energies of central $\mathrm{Cu}$ and $\mathrm{Ag}$ atoms with different neighboring compositions at $50 \mathrm{~K}$.

\begin{tabular}{cccccc}
\hline \hline \multicolumn{7}{c}{ Central Cu atoms } \\
\hline $\mathrm{Z}_{\mathrm{Cu}-\mathrm{Cu}}$ & $E(\mathrm{eV} /$ atom $)$ & $\mathrm{Z}_{\mathrm{Cu}-\mathrm{Zr}}$ & $E(\mathrm{eV} /$ atom $)$ & $\mathrm{Z}_{\mathrm{Cu}-\mathrm{Ag}}$ & $E(\mathrm{eV} /$ atom $)$ \\
\hline 2 & -3.761 & 4 & -3.596 & 0 & -3.724 \\
3 & -3.742 & 5 & -3.640 & 1 & -3.722 \\
4 & -3.721 & 6 & -3.678 & 2 & -3.720 \\
5 & -3.702 & 7 & -3.721 & 3 & -3.704 \\
6 & -3.673 & 8 & -3.764 & 4 & -3.692 \\
7 & -3.651 & 9 & -3.801 & 5 & -3.676 \\
\hline \multicolumn{7}{c}{} & \multicolumn{7}{c}{ Central $\mathrm{Ag}$ atoms } & \\
\hline $\mathrm{Z}_{\mathrm{Ag}-\mathrm{Cu}}$ & $E(\mathrm{eV} /$ atom $)$ & $\mathrm{Z}_{\mathrm{Ag}-\mathrm{Zr}}$ & $E(\mathrm{eV} /$ atom $)$ & $\mathrm{Z}_{\mathrm{Ag}-\mathrm{Ag}}$ & $E(\mathrm{eV} / \mathrm{atom})$ \\
\hline 3 & -2.896 & 3 & -2.855 & 0 & -2.792 \\
4 & -2.871 & 4 & -2.852 & 1 & -2.824 \\
5 & -2.840 & 5 & -2.855 & 2 & -2.850 \\
6 & -2.825 & 6 & -2.847 & 3 & -2.877 \\
7 & -2.814 & 7 & -2.857 & 4 & -2.891 \\
8 & -2.796 & 8 & -2.877 & 5 & -2.914 \\
\hline \hline
\end{tabular}


To study the microscopic origin of the CSRO formation, we correlated the energetic stability and local chemical compositions by monitoring the potential energy on each atom. The value of atomic potential energy could reveal the local energetic stability of atoms. Accordingly, atoms with low potential energy should correspond to stable conditions, while atoms with high potential energy are unfavorable. In this work, atoms of different species were categorized into various groups according to their nearestneighbor environments (or partial $\mathrm{CN}$ ). The partial $\mathrm{CN}$ clearly describes the neighboring chemical environment around a center atom. For instance, if a center $\mathrm{Cu}$ atom has 12 neighboring atoms (including $7 \mathrm{Zr}, 4 \mathrm{Cu}$ and $1 \mathrm{Ag}$ atoms), then the partial $\mathrm{CNs}$ for such atom should be $\mathrm{Z}_{\mathrm{Cu}-\mathrm{Zr}}=7, \mathrm{Z}_{\mathrm{Cu}-\mathrm{Cu}}=4$ and $\mathrm{Z}_{\mathrm{Cu}-\mathrm{Ag}}=1$. By averaging the potential energies on different groups of atoms, we can explain why some atomic pairs are favored, while others tend to be eliminated during the glass formation process. The correlations between potential energy and chemical composition are listed in Table 5.4. Impressively, those favored pairs agree well with the energetic stability of atoms. For example, $\mathrm{Cu}$ atoms with more neighboring $\mathrm{Zr}$ atoms possess lower potential energy, explaining why $\mathrm{Cu}-\mathrm{Zr}$ bonds can chemically stabilize the central $\mathrm{Cu}$ atoms in the system. In comparison, the $\mathrm{Cu}-\mathrm{Ag}$ and $\mathrm{Cu}-\mathrm{Cu}$ interactions destabilize the central $\mathrm{Cu}$ atoms, as these two pairs follow opposite trends of the $\mathrm{Cu}-\mathrm{Zr}$ bonds. Likewise, the energetic states of $\mathrm{Ag}$ atoms also represent the chemical affinity of different pairs. Therefore, the CSRO development in MGs mainly results from the tendency of atoms to form glasses with higher stability.

Due to the strong preference of $\mathrm{Ag}-\mathrm{Ag}$ pairs and avoidance of $\mathrm{Cu}-\mathrm{Ag}$ pairs, $\mathrm{Cu}$ and $\mathrm{Ag}$ atoms are inhomogeneously distributed in this model. Such nanoscale phase separation is commonly observed in some multicomponent MGs $[158,160]$. In accordance with previous work [167], this system could be separated into $\mathrm{Cu}$ - and Ag-rich regions. These 
two regimes are expected to exhibit different dynamic and mechanical properties, as investigated in the following two sections.

\subsubsection{Relationships between chemical and dynamic heterogeneities}

To examine the correlations between chemical and dynamic heterogeneity, we carried out annealing processes at a constant temperature for the models. To ensure that the atomic mobility is relatively high during the relaxation, the annealing temperature selected $(800 \mathrm{~K})$ is a little bit above the glass transition temperature $(690 \mathrm{~K})$. In this work, we will focus on the dynamic and mechanical discrepancies between $\mathrm{Cu}$ - and $\mathrm{Ag}$ rich regimes. Similar to previous section, atoms are grouped according to their partial $\mathrm{CN}$, which determines the neighboring environment for each atom. According to the CSRO formation discussed in previous section, it can be expected that $\mathrm{Cu}$-rich regions are dominated by $\mathrm{Zr}-\mathrm{Cu}$ pairs, while Ag-rich regions are dominated by $\mathrm{Ag}-\mathrm{Ag}$ and $\mathrm{Zr}$ Ag pairs. 


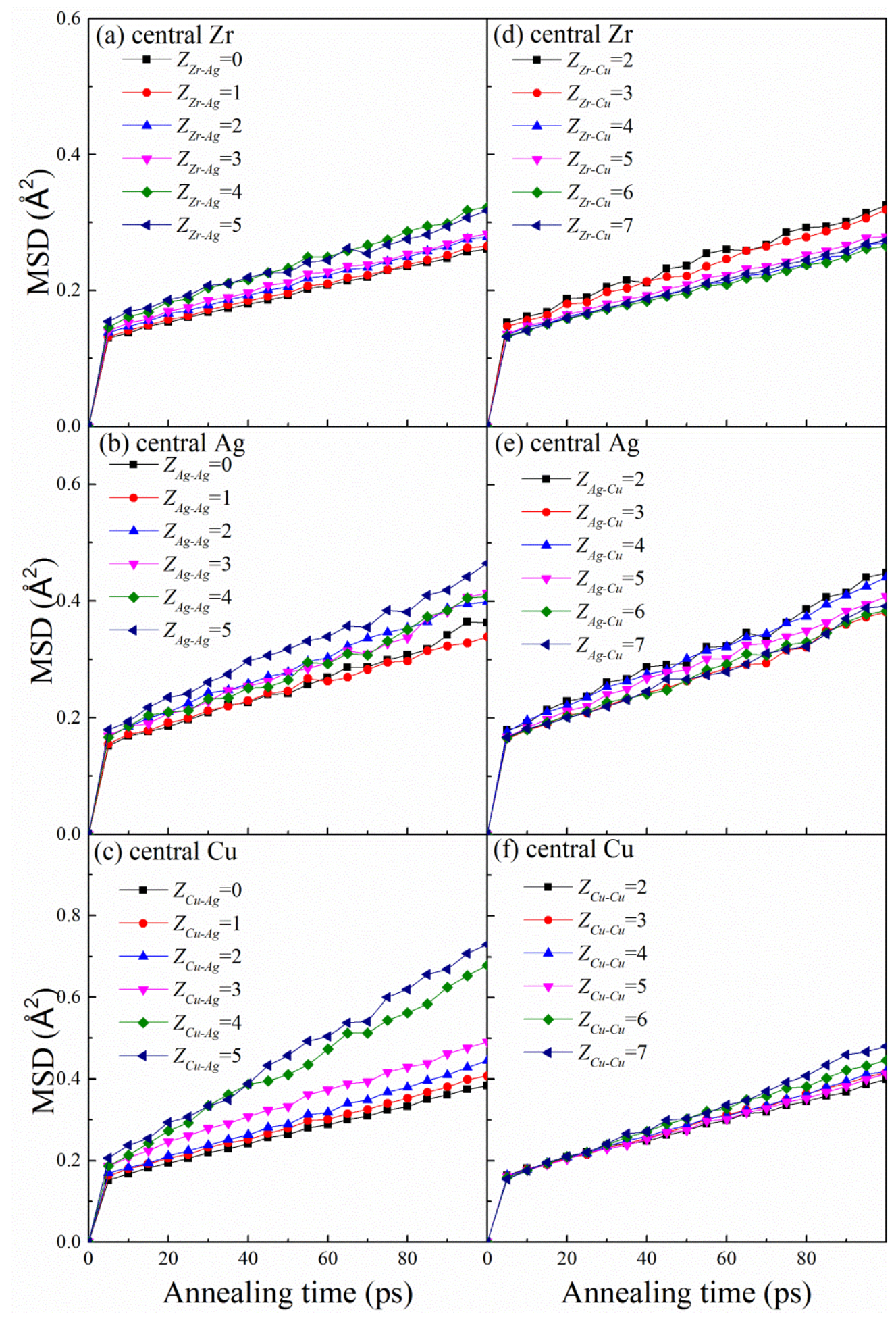

Figure 5.4 The dependence of atomic mobility ( $\mathrm{Zr}, \mathrm{Cu}$ and $\mathrm{Ag}$ atoms) on neighboring chemical compositions, showing the relationship between chemical and dynamic heterogeneities. Atoms in Ag-rich or $\mathrm{Cu}$-poor regions are mostly observed to exhibit faster dynamics. 
MSD has been extensively used to quantify the local dynamic properties of atoms, since it directly reveals the atomic mobility in supercooled liquids [161]. The non-Gaussian parameter [73] was firstly calculated to obtain the "maximum non-Gaussian time". This time is commonly adopted to investigate the dynamic heterogeneity in supercooled liquids. Accordingly, the time interval adopted in our simulations was 100 ps. Twenty simulation runs (annealing at $800 \mathrm{~K}$ ) were then implemented and averaged, with the same initial configuration but different distribution of initial momenta of atoms. The MSD evolutions of atoms with different chemical environment are plotted in Figures 5.4(a) to (f). $\mathrm{Zr}, \mathrm{Cu}$ and $\mathrm{Ag}$ atoms are categorized according to their partial $\mathrm{CN}$ with respect to $\mathrm{Ag}$ (Figures 5.4(a) to (c)) and $\mathrm{Cu}$ (Figures 5.4(e) to (f)) atoms. As seen in these figures, atoms in Ag-rich regimes generally exhibit fast dynamics, while $\mathrm{Zr}$ atoms in $\mathrm{Cu}$-rich regions exhibit slow dynamics. Using Figures 5.4(a) and (d) for example, $\mathrm{Zr}$ atoms in $\mathrm{Cu}$-rich and $\mathrm{Ag}$-poor environments are found to exhibit lower MSD during the annealing process. These findings are consistent with the work by Fujita et al. [167]. A possible explanation is that the local activation energies vary with the chemical heterogeneities in $\mathrm{Zr}-\mathrm{Cu}-\mathrm{Ag}$ MGs [175]. Therefore, atoms in Ag-rich regions are easier to be activated under thermal perturbations, showing faster dynamics than those in $\mathrm{Cu}$ rich regions. On the other hand, according to Figures 5.4(e) and (f), the central Ag (or $\mathrm{Cu}$ ) atoms in $\mathrm{Cu}$-rich environments exhibit similar dynamics with those in $\mathrm{Cu}$-poor regions. Such phenomenon may originate from the energetic instability of $\mathrm{Ag}-\mathrm{Cu}$ and $\mathrm{Cu}-\mathrm{Cu}$ pairs, which is mentioned in the above section. Based on the MSD analyses, the intrinsic correlations between the chemical and dynamic heterogeneities have been detected in the $\mathrm{Zr}_{45} \mathrm{Cu}_{45} \mathrm{Ag}_{10}$ glasses. 


\subsubsection{Relationships between chemical and mechanical heterogeneities}

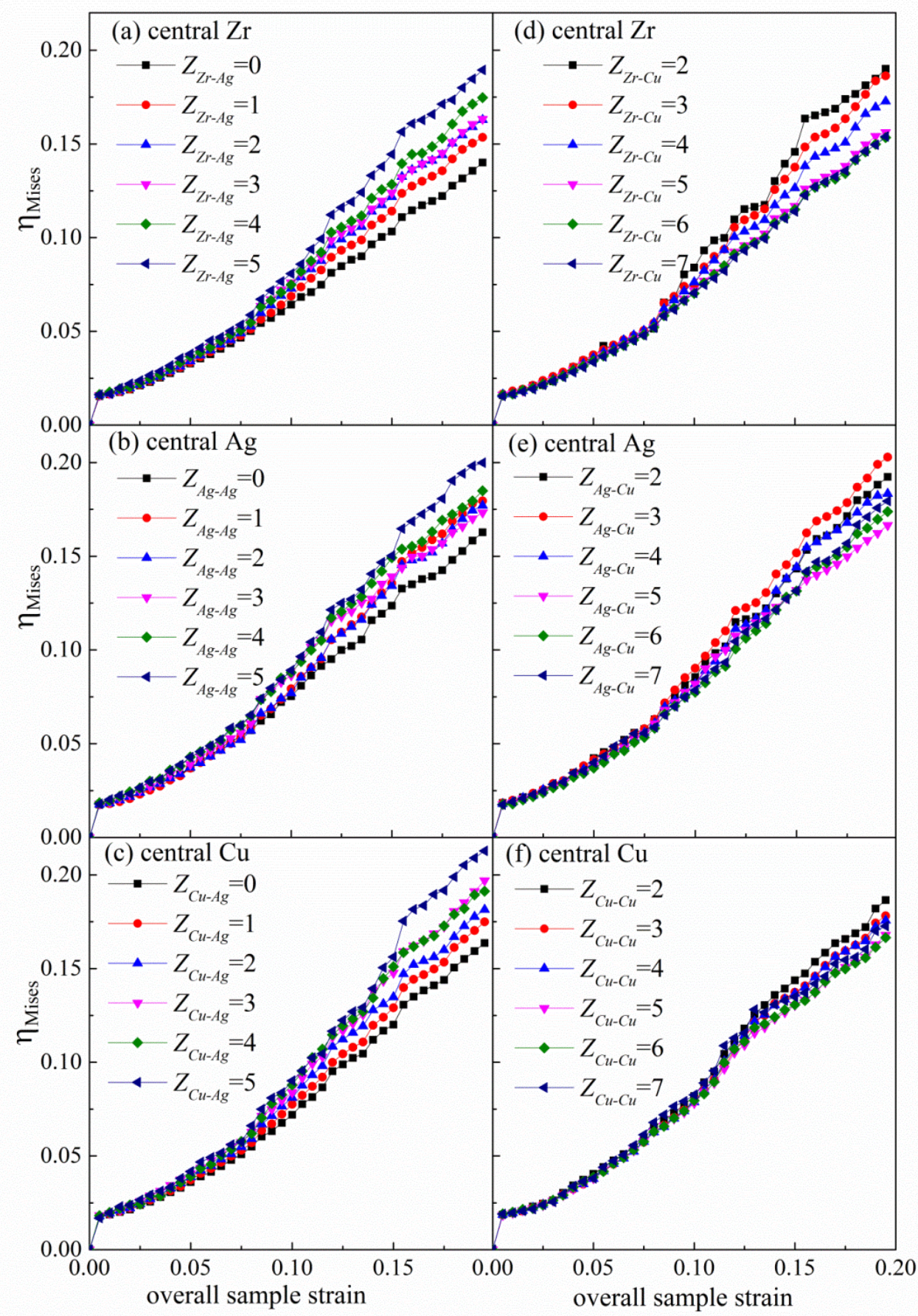

Figure 5.5 The dependence of local shear resistance $(\mathrm{Zr}, \mathrm{Cu}$ and $\mathrm{Ag}$ atoms) on neighboring chemical compositions, showing the relationship between chemical and mechanical heterogeneities. Similarly, atoms in Ag-rich or $\mathrm{Cu}$-poor regions exhibit lower resistance to shear deformations. 
Quantification of von Mises shear strain can reflect the variation of local stiffness in MGs. Typically, atoms exhibit higher von Mises shear strain are considered as fertile sites for shear transformation events. By computing the von Mises shear strain, the strong connections between chemical and mechanical heterogeneity have also been found, as shown in Figures 5.5 (a) to (f). Interestingly, atoms with more neighboring $\mathrm{Cu}$ and less Ag particles exhibit smaller shear strain during the pure shear loading process. We thus can conclude that atoms in Ag-rich regions are likely to be activated for plastic deformation, whereas atoms in $\mathrm{Cu}$-rich regions show elastic behaviors (solid-like). Similarly, the mechanical heterogeneity in $\mathrm{Cu}-\mathrm{Zr}-\mathrm{Ag}$ MGs may result from the locally varying activation energies, which is induced by the nanoscale phase separation of $\mathrm{Cu}$ and Ag-enriched regimes. Based on these results, we figured out the correlation between chemical, dynamic and mechanical heterogeneities in the $\mathrm{Zr}_{45} \mathrm{Cu}_{45} \mathrm{Ag}_{10}$ glasses. Atoms in Ag-rich regions are easier to be activated by external stimuli (thermal perturbations or applied stresses). Therefore, our findings may explain the micromechanisms underlying the physical and mechanical properties of realistic phase-separated BMGs.

\subsection{Summary}

In summary, we investigated the CSRO formation and its influences on local dynamic/mechanical properties in $\mathrm{Zr}_{45} \mathrm{Cu}_{45} \mathrm{Ag}_{10} \mathrm{MGs}$ by using classical MD simulations. Our analyses of Warren-Cowley parameters reveal that rapid growth of CSRO in the supercooled region. In addition, the reduction of effective cooling rates can significantly prompt the CSRO in MGs. By calculating the atomic potential energy, it is found that the formation of CSRO is directly linked with the local energetic stability. Furthermore, the correlations between chemical, dynamic and mechanical heterogeneities were captured by simulating annealing and shear loading processes. The $\mathrm{Cu}$-rich regions 
exhibit slower dynamics and higher shear resistance under external stimuli, while Agrich regions are likely to be activated. 


\section{CHAPTER 6 CONCLUSIONS AND FUTURE WORK}

This chapter summarizes the simulation results in this $\mathrm{PhD}$ work. In addition, it highlights the main contribution of this research work. Last but not least, the future work that can be undertaken towards the extension of this $\mathrm{PhD}$ work is presented.

\subsection{Conclusions}

The $\mathrm{PhD}$ research work presents an investigation of $\mathrm{MD}$ simulation on the nanostructures and structure-property relationship of MGs.

The structural defects play a key role in controlling the physical and mechanical behaviors of materials. For amorphous alloys, various parameters (e.g., free volume, Voronoi index) have been extensively employed to study the structural defects and structure-property relationships. Unfortunately, these two parameters are limited to particular types of glass-forming systems. Therefore in chapter 3, atomic level stress is used as a system-independent criterion to define and investigate the structural defects in $\mathrm{Cu}-\mathrm{Zr}$ MGs. By analyzing samples with different compositions, the atomic-level stress fluctuations are closely linked with the local kinetic and mechanical responses in modeled $\mathrm{Cu}_{\mathrm{x}} \mathrm{Zr}_{100-\mathrm{x}}$ MGs. Other than Voronoi index, atomic-level stress is proven to be a generic parameter to identify structural defects in different glasses. In addition, $\gamma$-defects with excessive shear stress exhibit faster dynamics than solid-like atoms. By performing shear deformation processes, it is also proposed that that $\gamma$-defects are fertile sites for local inelastic deformation.

In recent decades, researchers have carried out numerous simulations devoted to the characterization of TSRO in MGs. Nevertheless, a clear understanding of the MRO is still necessary to understand the mechanical heterogeneity of amorphous alloys. Chapter 
4 examines the relationships between local fivefold symmetry and medium-range structures in $\mathrm{Zr}_{46} \mathrm{Cu}_{46} \mathrm{Al}_{8}$ MGs. Based on the Voronoi tessellation techniques, the results provide insights into the structural and mechanical heterogeneity of MGs. In addition to the solid-like backbones formed by HLFFS atoms, there also exist networks formed by LLFFS atoms, representing the liquid-like (unstable) regions in this sample. Our calculation of atomic level stress reveals the correlations between atomic shear stress and local fivefold symmetry. Additionally, the spatial connections of atoms (mediumrange structures) are significantly affected by the atomic level stress. These findings indicate the microscopic origins of structural heterogeneity in MGs. The mechanical heterogeneity of MGs was investigated by performing a pure shear loading process. It is found that LLFFS atoms tend to initiate plastic flow, while HLFFS entities are resistant to plastic deformation. Such observation validates our assumption that the HLFFS and LLFFS networks respectively represent the solid- and liquid-like regions in $\mathrm{Zr}_{46} \mathrm{Cu}_{46} \mathrm{Al}_{8}$ glasses.

Similar to the TSRO, CSRO is another key factor determining the dynamic and mechanical features of MGs. For instance, the plasticity enhancement of various MGs is proposed to stem from the phase separation phenomena. Chapter 5 studies the CSRO formation and its links to dynamic/mechanical heterogeneities in $\mathrm{Zr}_{45} \mathrm{Cu}_{45} \mathrm{Ag}_{10}$ MGs. The Warren-Cowley parameter evolution reveals the rapid growth of CSRO in the supercooled region. Similar to the TSRO development reported in previous studies, the CSRO exhibits cooling history dependence. The CSRO development is directly attributed to the local energetic stability. Additionally, the correlations between chemical, dynamic and mechanical heterogeneities were captured by simulating annealing and shear loading processes. The $\mathrm{Cu}$-enriched regions are highly resistant to external stimuli and Ag-enriched regions in contrast are likely to be activated. 


\subsection{Main contributions arising from this thesis}

The work in this thesis mainly addresses the microstructure and structure-property relationships in MGs. The main contributions arising from this thesis are listed as follows:

(1) Validating the generic applicability of atomic-level stress in identifying the structural defects in amorphous alloys. Atoms with excessive stress exhibit dynamic and mechanical instability under external stimuli.

(2) Demonstrating the correlations between local fivefold symmetry and medium-range structures in $\mathrm{Zr}-\mathrm{Cu}-\mathrm{Al}$ glass. Similar to the solid-like backbones formed by HLFFS atoms, there also exist networks formed by LLFFS atoms.

(3) Examining the dependence of local fivefold symmetry on atomic-level shear stress. It is found that HLFFS atoms typically possess smaller atomic shear stress, which may explain the mechanical discrepancies between HLFFS and LLFFS atoms. The HLFFS and LLFFS respectively exhibit solid- and liquid-like behaviors under shear deformations.

(4) Investigating the rapid growth of CSRO in supercooled region. Similar to the TSRO, the CSRO development also significantly depends on the cooling histories. The CSRO development is directly linked with the energetic stability.

(5) The effects of CSRO on the dynamic and mechanical heterogeneities in $\mathrm{Zr}-\mathrm{Cu}-\mathrm{Ag}$ MGs. It is concluded that Ag-enriched regions exhibit instability during annealing and deformation processes.

\subsection{Future work}

The work in this thesis addresses the nanostructures and structure-property relationships in MGs. Although significant progress has been made to examine the structure-dynamic 
and structure-mechanical correlations in amorphous alloys, there still remains interesting and challenging issues for further explorations.

\subsubsection{Crystal-like order in multicomponent glasses}

As aforementioned, there exist two types of medium-range networks in $\mathrm{Zr}_{46} \mathrm{Cu}_{46} \mathrm{Al}_{8}$ glasses. The HLFFS and LLFFS networks respectively serve as solid-like and liquid-like regions under shear deformation. Previous RMC simulations revealed the crystal- and icosahedral-like MRO in $\mathrm{Zr}-\mathrm{Cu}-\mathrm{Al}$ MGs [126, 148]. Following their studies, atoms with different degree of fivefold symmetry are grouped into three categories: crystal-, icosahedral-like and mixed polyhedra. Similarly, both crystal- and icosahedral-like atoms tend to agglomerate at medium range scale, forming nanometer-scale superclusters. Interestingly, the crystal-like superclusters exhibit orderliness and rotational symmetry in those annealed models, which may represent the commencement of nucleation and crystallization in glasses. Additionally, the fraction of crystal-like atoms increases upon annealing in $\mathrm{Zr}_{50} \mathrm{Cu}_{35} \mathrm{Al}_{15}$ glass, which exhibits an opposite trend in $\mathrm{Zr}_{50} \mathrm{Cu}_{45} \mathrm{Al}_{5} \mathrm{MG}$. Due to the rotational symmetry founded in crystal-like MRO, one may expect the mechanical stability of crystal-like superclusters in $\mathrm{Zr}-\mathrm{Cu}-\mathrm{Al} \mathrm{MGs}$. On the other hand, our simulation results suggest the low stiffness of HLFFS networks, which seems to be controversial with the RMC models. Accordingly, a comprehensive investigation on the crystal-like order is necessary for understanding its structural and mechanical features. Further investigations may involve various issues including: (1) the structural and mechanical comparisons between HLFFS networks and crystal-like MRO; (2) the dynamic and mechanical heterogeneities in relation with crystal-like and icosahedral-like MRO; (3) the development of crystal-like MRO (or crystallization) in MGs upon annealing. 


\subsubsection{Various factors affecting the mechanical behaviors of metallic glass matrix nanocomposites}

The tailorable mechanical properties of MGMCs, strength, hardness, plasticity and toughness, render them as attractive materials for widespread engineering applications. With the introduction of either micro- or nano-sized crystallites, significant ductility is observed for various MGMCs [100, 176-178]. The improved ductility of MGMCs is mainly attributed to the initiation of multiple STZs at glass-crystal interfaces and the suppression of shear band propagation [2]. The plasticity improvement and work hardening of MGMCs containing micro-sized precipitates is typically ascribed to the martensitic transformation (MT) of ductile dendrites [179-181]. With a high volume fraction of ductile phases, both STZ initiations at the interface and blocking of shear band extension are responsible for the improved ductility [182]. Compared to microsized precipitates, nanoscale reinforcements are nearly perfect crystals without internal defects. Hence the deformation mechanisms of MGMCs containing nanoprecipitates are supposed to be quite different. The primary reason for the improved ductility is believed to stem from the activation of multiple STZs at glass-crystal interfaces, which is caused by the elastoplastic mismatch between nanocrystals and glass matrix [183, 184]. Nevertheless, the underlying mechanisms of the STZ activations have not been fully understood. A clear understanding of the mechanical properties requires further exploration on the deformation behaviors of MGMCs with nanoprecipitates. In particular, structural factors (crystal orientations, size, distribution, volume fraction and etc.) affecting the shear band initiation and propagation will be discussed and studied in our further studies. For instance, the mechanical behaviors of nanocrystals are apparently orientation dependent. Accordingly, the shear band initiation of nanocomposites could be closely correlated with the crystal orientation. The focus of our 
future work is on the shear band initiations and propagations in MGMCs containing nanocrystals. 


\section{REFERENCES}

[1] W. Klement, R. Willens, P. Duwez, Non-crystalline structure in solidified goldsilicon alloys, (1960).

[2] C.A. Schuh, T.C. Hufnagel, U. Ramamurty, Mechanical behavior of amorphous alloys, Acta Materialia, 55 (2007) 4067-4109.

[3] J. Eckert, J. Das, S. Pauly, C. Duhamel, Mechanical properties of bulk metallic glasses and composites, Journal of materials research, 22 (2007) 285-301.

[4] P. Chaudhari, D. Turnbull, Structure and properties of metallic glasses, Science, 199 (1978) 11-21.

[5] H. Chen, Glassy metals, Reports on Progress in Physics, 43 (1980) 353.

[6] A.L. Greer, Metallic glasses, Science, 267 (1995) 1947.

[7] W.L. Johnson, Bulk glass-forming metallic alloys: Science and technology, MRS bulletin, 24 (1999) 42-56.

[8] A. Inoue, Stabilization of metallic supercooled liquid and bulk amorphous alloys, Acta materialia, 48 (2000) 279-306.

[9] A. Inoue, A. Takeuchi, Recent progress in bulk glassy, nanoquasicrystalline and nanocrystalline alloys, Materials Science and Engineering: A, 375 (2004) 16-30.

[10] D.M. Herlach, R. Cochrane, I. Egry, H. Fecht, A. Greer, Containerless processing in the study of metallic melts and their solidification, International Materials Reviews, 38 (1993) 273-347.

[11] M. Chen, Mechanical behavior of metallic glasses: microscopic understanding of strength and ductility, Annu. Rev. Mater. Res., 38 (2008) 445-469.

[12] Y.Q. Cheng, E. Ma, Atomic-level structure and structure-property relationship in metallic glasses, Progress in Materials Science, 56 (2011) 379-473. 
[13] W.-H. Wang, C. Dong, C. Shek, Bulk metallic glasses, Materials Science and Engineering: R: Reports, 44 (2004) 45-89.

[14] H. Chen, S. Ferris, E. Gyorgy, H. Leamy, R. Sherwood, Field heat treatment of ferromagnetic metallic glasses, Applied Physics Letters, 26 (1975) 405-406.

[15] D. Turnbull, Under what conditions can a glass be formed?, Contemporary physics, 10 (1969) 473-488.

[16] H. Chen, D. Turnbull, Evidence of a glass-liquid transition in a gold-germaniumsilicon alloy, The Journal of Chemical Physics, 48 (1968) 2560-2571.

[17] A. Inoue, C. Fan, J. Saida, T. Zhang, High-strength Zr-based bulk amorphous alloys containing nanocrystalline and nanoquasicrystalline particles, Science and Technology of Advanced Materials, 1 (2000) 73-86.

[18] H. Chen, Thermodynamic considerations on the formation and stability of metallic glasses, Acta Metallurgica, 22 (1974) 1505-1511.

[19] A. Drehman, A. Greer, D. Turnbull, Bulk formation of a metallic glass: $\mathrm{Pd}_{40} \mathrm{Ni}_{40} \mathrm{P}_{20}$, Applied Physics Letters, 41 (1982) 716-717.

[20] A. Inoue, T. Zhang, T. Masumoto, Al-La-Ni amorphous alloys with a wide supercooled liquid region, Materials Transactions, JIM, 30 (1989) 965-972.

[21] A. Inoue, T. Zhang, Thermal stability and glass-forming ability of amorphous NdAl-TM $(\mathrm{TM}=\mathrm{Fe}, \mathrm{Co}, \mathrm{Ni}$ or $\mathrm{Cu})$ alloys, Materials Science and Engineering: A, 226 (1997) 393-396.

[22] Y. Zhang, H. Tan, Y. Li, Bulk glass formation of $12 \mathrm{~mm}$ rod in $\mathrm{La}-\mathrm{Cu}-\mathrm{Ni}-\mathrm{Al}$ alloys, Materials Science and Engineering: A, 375 (2004) 436-439.

[23] A. Inoue, T. Zhang, N. Nishiyama, K. Ohba, T. Masumoto, Preparation of $16 \mathrm{~mm}$ diameter rod of amorphous $\mathrm{Zr}_{65} \mathrm{Al}_{7.5} \mathrm{Ni}_{10} \mathrm{Cu}_{17.5}$ alloy, Materials Transactions, JIM, 34 (1993) 1234-1237. 
[24] A. Inoue, N. Nishiyama, H. Kimura, Preparation and thermal stability of bulk amorphous $\mathrm{Pd}_{40} \mathrm{Cu}_{30} \mathrm{Ni}_{10} \mathrm{P}_{20}$ alloy cylinder of $72 \mathrm{~mm}$ in diameter, Materials Transactions, JIM, 38 (1997) 179-183.

[25] A. Peker, W.L. Johnson, A highly processable metallic glass: $\mathrm{Zr}_{41.2} \mathrm{Ti}_{13.8} \mathrm{Cu}_{12.5} \mathrm{Ni}_{10}$. ${ }_{0} \mathrm{Be}_{22.5}$, Applied Physics Letters, 63 (1993) 2342-2344.

[26] Y. Zeng, N. Nishiyama, T. Yamamoto, A. Inoue, Ni-rich bulk metallic glasses with high glass-forming ability and good metallic properties, Materials transactions, 50 (2009) 2441-2445.

[27] V. Ponnambalam, S.J. Poon, G.J. Shiflet, Fe-based bulk metallic glasses with diameter thickness larger than one centimeter, Journal of Materials Research, 19 (2004) $1320-1323$.

[28] D. Xu, G. Duan, W.L. Johnson, Unusual glass-forming ability of bulk amorphous alloys based on ordinary metal copper, Physical review letters, 92 (2004) 245504.

[29] F. Guo, H.-J. Wang, S.J. Poon, G.J. Shiflet, Ductile titanium-based glassy alloy ingots, Applied Physics Letters, 86 (2005) 1907.

[30] N. Nishiyama, K. Takenaka, H. Miura, N. Saidoh, Y. Zeng, A. Inoue, The world's biggest glassy alloy ever made, Intermetallics, 30 (2012) 19-24.

[31] J. Qiao, H. Jia, P.K. Liaw, Metallic glass matrix composites, Materials Science and Engineering: R: Reports, 100 (2016) 1-69.

[32] W. Gotze, L. Sjogren, Relaxation processes in supercooled liquids, Reports on progress in Physics, 55 (1992) 241.

[33] M.D. Ediger, C. Angell, S.R. Nagel, Supercooled liquids and glasses, The journal of physical chemistry, 100 (1996) 13200-13212.

[34] P.G. Debenedetti, F.H. Stillinger, Supercooled liquids and the glass transition, Nature, 410 (2001) 259-267. 
[35] A.L. Greer, Confusion by design, Nature: International weekly journal of science, 366 (1993) 303-304.

[36] A. Inoue, High strength bulk amorphous alloys with low critical cooling rates (overview), Materials Transactions, JIM, 36 (1995) 866-875.

[37] R. Busch, J. Schroers, W. Wang, Thermodynamics and kinetics of bulk metallic glass, Mrs Bulletin, 32 (2007) 620-623.

[38] C.A. Angell, Formation of glasses from liquids and biopolymers, Science, 267 (1995) 1924-1935.

[39] C.A. Angell, Spectroscopy simulation and scattering, and the medium range order problem in glass, Journal of Non-Crystalline Solids, 73 (1985) 1-17.

[40] J.J. Gilman, Mechanical behavior of metallic glasses, Journal of applied Physics, 46 (1975) 1625-1633.

[41] M. Ashby, A. Greer, Metallic glasses as structural materials, Scripta Materialia, 54 (2006) 321-326.

[42] A.R. Yavari, J. Lewandowski, J. Eckert, Mechanical properties of bulk metallic glasses, Mrs Bulletin, 32 (2007) 635-638.

[43] A. Greer, Y. Cheng, E. Ma, Shear bands in metallic glasses, Materials Science and Engineering: R: Reports, 74 (2013) 71-132.

[44] M.M. Trexler, N.N. Thadhani, Mechanical properties of bulk metallic glasses, Progress in Materials Science, 55 (2010) 759-839.

[45] A. Argon, Plastic deformation in metallic glasses, Acta metallurgica, 27 (1979) 4758.

[46] C.A. Schuh, A.C. Lund, Atomistic basis for the plastic yield criterion of metallic glass, Nature materials, 2 (2003) 449-452. 
[47] A. Lund, C. Schuh, The Mohr-Coulomb criterion from unit shear processes in metallic glass, Intermetallics, 12 (2004) 1159-1165.

[48] F. Spaepen, A microscopic mechanism for steady state inhomogeneous flow in metallic glasses, Acta metallurgica, 25 (1977) 407-415.

[49] M. Heggen, F. Spaepen, M. Feuerbacher, Creation and annihilation of free volume during homogeneous flow of a metallic glass, Journal of Applied Physics, 97 (2005) 33506-33506.

[50] W. Wang, Elastic moduli and behaviors of metallic glasses, Journal of noncrystalline solids, 351 (2005) 1481-1485.

[51] J. Wang, W. Wang, H. Yu, H. Bai, Correlations between elastic moduli and molar volume in metallic glasses, Applied Physics Letters, 94 (2009) 121904.

[52] T.H. Courtney, Mechanical behavior of materials, Waveland Press, 2005.

[53] A. Granato, The specific heat of simple liquids, Journal of non-crystalline solids, 307 (2002) 376-386.

[54] D. Safarik, R. Schwarz, Elastic constants of amorphous and single-crystal $\mathrm{Pd}_{40} \mathrm{Cu}_{40} \mathrm{P}_{20}$, Acta Materialia, 55 (2007) 5736-5746.

[55] S. Khonik, A. Granato, D. Joncich, A. Pompe, V. Khonik, Evidence of distributed interstitialcy-like relaxation of the shear modulus due to structural relaxation of metallic glasses, Physical review letters, 100 (2008) 065501.

[56] Y. Suzuki, T. Egami, Shear deformation of glassy metals: Breakdown of Cauchy relationship and anelasticity, Journal of Non-Crystalline Solids, 75 (1985) 361-366.

[57] G. Knuyt, L. De Schepper, L. Stals, Calculation of elastic constants for an amorphous metal and the influence of relaxation, Journal of Physics F: Metal Physics, 16 (1986) 1989. 
[58] R. Conner, H. Choi-Yim, W. Johnson, Mechanical properties of $\mathrm{Zr}_{57} \mathrm{Nb}_{5} \mathrm{Al}_{10} \mathrm{Cu}_{15.4} \mathrm{Ni}_{12.6}$ metallic glass matrix particulate composites, Journal of Materials Research, 14 (1999) 3292-3297.

[59] A. Inoue, B. Shen, C. Chang, Fe-and Co-based bulk glassy alloys with ultrahigh strength of over 4000MPa, Intermetallics, 14 (2006) 936-944.

[60] L. Xing, T. Hufnagel, K. Ramesh, Plastic deformation of bulk amorphous alloys, in: MRS Proceedings, Cambridge Univ Press, 2000, pp. L11. 17.

[61] C. Schuh, T. Nieh, A nanoindentation study of serrated flow in bulk metallic glasses, Acta Materialia, 51 (2003) 87-99.

[62] C. Pampillo, H. Chen, Comprehensive plastic deformation of a bulk metallic glass, Materials Science and Engineering, 13 (1974) 181-188.

[63] T. Mukai, T. Nieh, Y. Kawamura, A. Inoue, K. Higashi, Effect of strain rate on compressive behavior of a $\mathrm{Pd}_{40} \mathrm{Ni}_{40} \mathrm{P}_{20}$ bulk metallic glass, Intermetallics, 10 (2002) 1071-1077.

[64] H.W. Sheng, W.K. Luo, F.M. Alamgir, J.M. Bai, E. Ma, Atomic packing and shortto-medium-range order in metallic glasses, Nature, 439 (2006) 419-425.

[65] M. Fdez-Gubieda, I. Orue, F. Plazaola, J. Barandiarán, Evidence of strong shortrange order in $\left(\mathrm{Fe}_{0.2} \mathrm{Co}_{0.8}\right)_{75} \mathrm{Si}_{\mathrm{x}} \mathrm{B}_{25-\mathrm{x}}$ amorphous alloys from EXAFS spectroscopy, Physical Review B, 53 (1996) 620.

[66] T. Egami, Atomic level stresses, Progress in Materials Science, 56 (2011) 637-653.

[67] D.B. Miracle, A structural model for metallic glasses, Nature materials, 3 (2004) 697-702.

[68] C.C. Wang, C.H. Wong, Short-to-medium range order of $\mathrm{Al}-\mathrm{Mg}$ metallic glasses studied by molecular dynamics simulations, Journal of alloys and compounds, 509 (2011) 10222-10229. 
[69] H. Sheng, Y. Cheng, P. Lee, S. Shastri, E. Ma, Atomic packing in multicomponent aluminum-based metallic glasses, Acta Materialia, 56 (2008) 6264-6272.

[70] C.C. Wang, C.H. Wong, Different icosahedra in metallic glasses: Stability and response to shear transformation, Scripta Materialia, 66 (2012) 610-613.

[71] Y.Q. Cheng, E. Ma, H.W. Sheng, Atomic level structure in multicomponent bulk metallic glass, Physical review letters, 102 (2009) 245501.

[72] J. Ding, Y.Q. Cheng, E. Ma, Full icosahedra dominate local order in $\mathrm{Cu}<\mathrm{sub}>$ $64</$ sub $>\mathrm{Zr}<\mathrm{sub}>34</$ sub $>$ metallic glass and supercooled liquid, Acta Materialia, 69 (2014) 343-354.

[73] Y.Q. Cheng, H.W. Sheng, E. Ma, Relationship between structure, dynamics, and mechanical properties in metallic glass-forming alloys, Physical Review B, 78 (2008) 014207.

[74] W. Kob, C. Donati, S.J. Plimpton, P.H. Poole, S.C. Glotzer, Dynamical heterogeneities in a supercooled Lennard-Jones liquid, Physical review letters, 79 (1997) 2827.

[75] C. Donati, S.C. Glotzer, P.H. Poole, W. Kob, S.J. Plimpton, Spatial correlations of mobility and immobility in a glass-forming Lennard-Jones liquid, Physical Review E, 60 (1999) 3107.

[76] H. Sillescu, Heterogeneity at the glass transition: a review, Journal of NonCrystalline Solids, 243 (1999) 81-108.

[77] J.P. Doye, L. Meyer, Mapping the magic numbers in binary Lennard-Jones clusters, Physical review letters, 95 (2005) 063401.

[78] N. Jakse, A. Pasturel, Local order and dynamic properties of liquid and undercooled $\mathrm{Cu}_{\mathrm{x}} \mathrm{Zr}_{1-\mathrm{x}}$ alloys by ab initio molecular dynamics, Physical Review B, 78 (2008) 214204. 
[79] M. Mendelev, M. Kramer, R. Ott, D. Sordelet, Molecular dynamics simulation of diffusion in supercooled Cu-Zr alloys, Philosophical Magazine, 89 (2009) 109-126.

[80] Y.Q. Cheng, A.J. Cao, H.W. Sheng, E. Ma, Local order influences initiation of plastic flow in metallic glass: Effects of alloy composition and sample cooling history, Acta Materialia, 56 (2008) 5263-5275.

[81] M. Tsamados, A. Tanguy, C. Goldenberg, J.-L. Barrat, Local elasticity map and plasticity in a model Lennard-Jones glass, Physical Review E, 80 (2009) 026112.

[82] S. Mayr, Relaxation kinetics and mechanical stability of metallic glasses and supercooled melts, Physical Review B, 79 (2009) 060201.

[83] Y. Shi, M.L. Falk, Structural transformation and localization during simulated nanoindentation of a noncrystalline metal film, Applied Physics Letters, 86 (2005) 011914.

[84] Y. Shi, M.L. Falk, Atomic-scale simulations of strain localization in threedimensional model amorphous solids, Physical Review B, 73 (2006) 214201.

[85] C.E. Lekka, A. Ibenskas, A. Yavari, G. Evangelakis, Tensile deformation accommodation in microscopic metallic glasses via subnanocluster reconstructions, Applied Physics Letters, 91 (2007) 4103.

[86] M. Wakeda, Y. Shibutani, Icosahedral clustering with medium-range order and local elastic properties of amorphous metals, Acta Materialia, 58 (2010) 3963-3969.

[87] P. Schall, D.A. Weitz, F. Spaepen, Structural rearrangements that govern flow in colloidal glasses, Science, 318 (2007) 1895-1899.

[88] A. Cao, Y. Cheng, E. Ma, Structural processes that initiate shear localization in metallic glass, Acta Materialia, 57 (2009) 5146-5155. 
[89] Y. Cheng, A. Cao, E. Ma, Correlation between the elastic modulus and the intrinsic plastic behavior of metallic glasses: The roles of atomic configuration and alloy composition, Acta materialia, 57 (2009) 3253-3267.

[90] Y. Zhang, A. Greer, Thickness of shear bands in metallic glasses, Applied physics letters, 89 (2006) 1907.

[91] H. Choi-Yim, R. Conner, F. Szuecs, W. Johnson, Processing, microstructure and properties of ductile metal particulate reinforced $\mathrm{Zr}_{57} \mathrm{Nb}_{5} \mathrm{Al}_{10} \mathrm{Cu}_{15.4} \mathrm{Ni}_{12.6}$ bulk metallic glass composites, Acta Materialia, 50 (2002) 2737-2745.

[92] Z. Bian, R.J. Wang, W.H. Wang, T. Zhang, A. Inoue, Carbon - Nanotube Reinforced Zr - Based Bulk Metallic Glass Composites and Their Properties, Advanced Functional Materials, 14 (2004) 55-63.

[93] H. Choi-Yim, R. Busch, U. Köster, W. Johnson, Synthesis and characterization of particulate reinforced $\mathrm{Zr}_{57} \mathrm{Nb}_{5} \mathrm{Al}_{10} \mathrm{Cu}_{15.4} \mathrm{Ni}_{12.6}$ bulk metallic glass composites, Acta Materialia, 47 (1999) 2455-2462.

[94] C. Hays, C. Kim, W.L. Johnson, Microstructure controlled shear band pattern formation and enhanced plasticity of bulk metallic glasses containing in situ formed ductile phase dendrite dispersions, Physical Review Letters, 84 (2000) 2901.

[95] H. Fu, H. Zhang, H. Wang, Q. Zhang, Z. Hu, Synthesis and mechanical properties of $\mathrm{Cu}$-based bulk metallic glass composites containing in-situ TiC particles, Scripta materialia, 52 (2005) 669-673.

[96] Y.-K. Xu, H. Ma, J. Xu, E. Ma, Mg-based bulk metallic glass composites with plasticity and gigapascal strength, Acta Materialia, 53 (2005) 1857-1866.

[97] H. Fu, H. Wang, H. Zhang, Z. Hu, In situ TiB-reinforced Cu-based bulk metallic glass composites, Scripta materialia, 54 (2006) 1961-1966. 
[98] H. Li, G. Subhash, L.J. Kecskes, R.J. Dowding, Mechanical behavior of tungsten preform reinforced bulk metallic glass composites, Materials Science and Engineering: A, 403 (2005) 134-143.

[99] A. Leonhard, L. Xing, M. Heilmaier, A. Gebert, J. Eckert, L. Schultz, Effect of crystalline precipitations on the mechanical behavior of bulk glass forming Zr-based alloys, Nanostructured Materials, 10 (1998) 805-817.

[100] M. Calin, J. Eckert, L. Schultz, Improved mechanical behavior of Cu-Ti-based bulk metallic glass by in situ formation of nanoscale precipitates, Scripta Materialia, 48 (2003) 653-658.

[101] Z. Bian, H. Kato, C. Qin, W. Zhang, A. Inoue, Cu-Hf-Ti-Ag-Ta bulk metallic glass composites and their properties, Acta materialia, 53 (2005) 2037-2048.

[102] U. Kühn, J. Eckert, N. Mattern, L. Schultz, Microstructure and mechanical properties of slowly cooled $\mathrm{Zr}-\mathrm{Nb}-\mathrm{Cu}-\mathrm{Ni}-\mathrm{Al}$ composites with ductile bcc phase, Materials Science and Engineering: A, 375 (2004) 322-326.

[103] M. Lee, Y. Li, C. Schuh, Effect of a controlled volume fraction of dendritic phases on tensile and compressive ductility in La-based metallic glass matrix composites, Acta Materialia, 52 (2004) 4121-4131.

[104] G. Kumar, A. Desai, J. Schroers, Bulk metallic glass: the smaller the better, Advanced materials, 23 (2011) 461-476.

[105] M. Chen, A brief overview of bulk metallic glasses, NPG Asia Materials, 3 (2011) $82-90$.

[106] H.L. Peng, M.Z. Li, W.H. Wang, C.Z. Wang, K.M. Ho, Effect of local structures and atomic packing on glass forming ability in $\mathrm{Cu}_{\mathrm{x}} \mathrm{Zr}_{100-\mathrm{x}}$ metallic glasses, Applied Physics Letters, 96 (2010) 021901. 
[107] A. Lagogianni, G. Almyras, C.E. Lekka, D. Papageorgiou, G. Evangelakis, Structural characteristics of $\mathrm{Cu}_{\mathrm{x}} \mathrm{Zr}_{100-\mathrm{x}}$ metallic glasses by Molecular Dynamics Simulations, Journal of Alloys and Compounds, 483 (2009) 658-661.

[108] G. Almyras, C.E. Lekka, N. Mattern, G. Evangelakis, On the microstructure of the $\mathrm{Cu}_{65} \mathrm{Zr}_{35}$ and $\mathrm{Cu} 35 \mathrm{Zr} 65$ metallic glasses, Scripta Materialia, 62 (2010) 33-36.

[109] M.P. Allen, D.J. Tildesley, Computer simulation of liquids, Oxford university press, 1989.

[110] J. Haile, Molecular dynamics simulation: Elementary methods, Computers in Physics, 7 (1993) 625-625.

[111] H.C. Andersen, Molecular dynamics simulations at constant pressure and/or temperature, The Journal of chemical physics, 72 (1980) 2384-2393.

[112] W.G. Hoover, Canonical dynamics: equilibrium phase-space distributions, Physical Review A, 31 (1985) 1695.

[113] L. Verlet, Computer" experiments" on classical fluids. I. Thermodynamical properties of Lennard-Jones molecules, Physical review, 159 (1967) 98.

[114] C.W. Gear, Numerical initial value problems in ordinary differential equations, Prentice Hall PTR, 1971.

[115] M.S. Daw, M.I. Baskes, Embedded-atom method: Derivation and application to impurities, surfaces, and other defects in metals, Physical Review B, 29 (1984) 6443.

[116] Y. Shi, M.L. Falk, Stress-induced structural transformation and shear banding during simulated nanoindentation of a metallic glass, Acta materialia, 55 (2007) 43174324.

[117] D. Miracle, A. Concustell, Y. Zhang, A. Yavari, A. Greer, Shear bands in metallic glasses: Size effects on thermal profiles, Acta Materialia, 59 (2011) 2831-2840. 
[118] Y.Q. Cheng, J. Ding, E. Ma, Local Topology vs. Atomic-Level Stresses as a Measure of Disorder: Correlating Structural Indicators for Metallic Glasses, Materials Research Letters, 1 (2013) 3-12.

[119] H.W. Sheng, E. Ma, M.J. Kramer, Relating Dynamic Properties to Atomic Structure in Metallic Glasses, JOM, 64 (2012) 856-881.

[120] K.-W. Park, J.-i. Jang, M. Wakeda, Y. Shibutani, J.-C. Lee, Atomic packing density and its influence on the properties of $\mathrm{Cu}-\mathrm{Zr}$ amorphous alloys, Scripta Materialia, 57 (2007) 805-808.

[121] M. Lee, C.M. Lee, K.R. Lee, E. Ma, J.C. Lee, Networked interpenetrating connections of icosahedra: Effects on shear transformations in metallic glass, Acta Materialia, 59 (2011) 159-170.

[122] Y. Sun, J. Shen, Icosahedral ordering in $\mathrm{Cu}_{60} \mathrm{Zr}_{40}$ metallic glass: Molecular dynamics simulations, Journal of Non-Crystalline Solids, 355 (2009) 1557-1560.

[123] H. Peng, M. Li, W. Wang, Structural signature of plastic deformation in metallic glasses, Physical review letters, 106 (2011) 135503.

[124] L. Ward, D. Miracle, W. Windl, O.N. Senkov, K. Flores, Structural evolution and kinetics in $\mathrm{Cu}-\mathrm{Zr}$ metallic liquids from molecular dynamics simulations, Physical Review B, 88 (2013) 134205.

[125] O. Senkov, D. Miracle, E. Barney, A. Hannon, Y. Cheng, E. Ma, Local atomic structure of Ca-Mg-Zn metallic glasses, Physical Review B, 82 (2010) 104206.

[126] P. Zhang, J.J. Maldonis, M. Besser, M. Kramer, P.M. Voyles, Medium-range structure and glass forming ability in $\mathrm{Zr}-\mathrm{Cu}-\mathrm{Al}$ bulk metallic glasses, Acta Materialia, 109 (2016) 103-114.

[127] D. Srolovitz, V. Vitek, T. Egami, An atomistic study of deformation of amorphous metals, Acta Metallurgica, 31 (1983) 335-352. 
[128] S. Plimpton, P. Crozier, A. Thompson, LAMMPS-large-scale atomic/molecular massively parallel simulator, Sandia National Laboratories, 18 (2007).

[129] M. Falk, J. Langer, Dynamics of viscoplastic deformation in amorphous solids, Physical Review E, 57 (1998) 7192.

[130] F. Shimizu, S. Ogata, J. Li, Theory of shear banding in metallic glasses and molecular dynamics calculations, Materials transactions, 48 (2007) 2923-2927.

[131] N. Mattern, P. Jóvári, I. Kaban, S. Gruner, A. Elsner, V. Kokotin, H. Franz, B. Beuneu, J. Eckert, Short-range order of $\mathrm{Cu}-\mathrm{Zr}$ metallic glasses, Journal of Alloys and Compounds, 485 (2009) 163-169.

[132] Y. Shi, M.L. Falk, Strain localization and percolation of stable structure in amorphous solids, Physical review letters, 95 (2005) 095502.

[133] M. Wakeda, Y. Shibutani, S. Ogata, J. Park, Relationship between local geometrical factors and mechanical properties for $\mathrm{Cu}-\mathrm{Zr}$ amorphous alloys, Intermetallics, 15 (2007) 139-144.

[134] Y.Q. Cheng, E. Ma, Configurational dependence of elastic modulus of metallic glass, Physical Review B, 80 (2009) 064104.

[135] F. Albano, N. Lacevic, M.L. Falk, S.C. Glotzer, Relating metallic glass mechanical properties to liquid structure, Materials Science and Engineering: A, 375 (2004) 671-674.

[136] H. Ke, J. Zeng, C. Liu, Y. Yang, Structure Heterogeneity in Metallic Glass: Modeling and Experiment, Journal of Materials Science \& Technology, (2014).

[137] J. Li, X. Chen, F. Huang, Inhomogeneous deformation in bulk metallic glasses: FEM analysis, Materials Science and Engineering: A, 620 (2015) 333-351. 
[138] Y. Liu, D. Wang, K. Nakajima, W. Zhang, A. Hirata, T. Nishi, A. Inoue, M. Chen, Characterization of nanoscale mechanical heterogeneity in a metallic glass by dynamic force microscopy, Physical review letters, 106 (2011) 125504.

[139] Y.Q. Cheng, E. Ma, Indicators of internal structural states for metallic glasses: Local order, free volume, and configurational potential energy, Applied Physics Letters, 93 (2008) 051910.

[140] Y. Zhang, H. Hahn, Quantification of the free volume in $\mathrm{Zr}_{45.0} \mathrm{Cu}_{39.3} \mathrm{Al}_{7.0} \mathrm{Ag}_{8.7}$ bulk metallic glasses subjected to plastic deformation by calorimetric and dilatometric measurements, Journal of alloys and compounds, 488 (2009) 65-71.

[141] H. Shao, Y. Xu, B. Shi, C. Yu, H. Hahn, H. Gleiter, J. Li, High density of shear bands and enhanced free volume induced in $\mathrm{Zr}_{70} \mathrm{Cu}_{20} \mathrm{Ni}_{10}$ metallic glass by high-energy ball milling, Journal of Alloys and Compounds, 548 (2013) 77-81.

[142] J. Ding, Y.Q. Cheng, E. Ma, Charge-transfer-enhanced prism-type local order in amorphous $\mathrm{Mg}_{65} \mathrm{Cu}_{25} \mathrm{Y}_{10}$ : Short-to-medium-range structural evolution underlying liquid fragility and heat capacity, Acta Materialia, 61 (2013) 3130-3140.

[143] Z. Sha, Y. Zhang, Y. Feng, Y. Li, Molecular dynamics studies of short to medium range order in $\mathrm{Cu}_{64} \mathrm{Zr}_{36}$ metallic glass, Journal of Alloys and Compounds, 509 (2011) 8319-8322.

[144] C.C. Wang, C.H. Wong, Interpenetrating networks in $\mathrm{Zr}-\mathrm{Cu}-\mathrm{Al}$ and $\mathrm{Zr}-\mathrm{Cu}$ metallic glasses, Intermetallics, 22 (2012) 13-16.

[145] Z.-C. Xie, T.-H. Gao, X.-T. Guo, X.-M. Qin, Q. Xie, Network connectivity in icosahedral medium-range order of metallic glass: A molecular dynamics simulation, Journal of Non-Crystalline Solids, 406 (2014) 31-36.

[146] J. Zemp, M. Celino, B. Schönfeld, J. Löffler, Icosahedral superclusters in $\mathrm{Cu}_{64} \mathrm{Zr}_{36}$ metallic glass, Physical Review B, 90 (2014) 144108. 
[147] M.Z. Li, C.Z. Wang, S.G. Hao, M.J. Kramer, K.M. Ho, Structural heterogeneity and medium-range order in $\mathrm{Zr}_{\mathrm{x}} \mathrm{Cu}_{100-\mathrm{x}}$ metallic glasses, Physical Review B, 80 (2009) 184201.

[148] J. Hwang, Z. Melgarejo, Y. Kalay, I. Kalay, M. Kramer, D. Stone, P. Voyles, Nanoscale structure and structural relaxation in $\mathrm{Zr}_{50} \mathrm{Cu}_{45} \mathrm{Al}_{5}$ bulk metallic glass, Physical review letters, 108 (2012) 195505.

[149] J. Ding, S. Patinet, M.L. Falk, Y. Cheng, E. Ma, Soft spots and their structural signature in a metallic glass, Proceedings of the National Academy of Sciences, 111 (2014) 14052-14056.

[150] S. Plimpton, Fast parallel algorithms for short-range molecular dynamics, Journal of computational physics, 117 (1995) 1-19.

[151] A. Stukowski, Visualization and analysis of atomistic simulation data with OVITO-the Open Visualization Tool, Modelling and Simulation in Materials Science and Engineering, 18 (2010) 015012.

[152] M. Leocmach, H. Tanaka, Roles of icosahedral and crystal-like order in the hard spheres glass transition, Nature communications, 3 (2012) 974.

[153] Y. Zhang, C.Z. Wang, F. Zhang, M.I. Mendelev, M.J. Kramer, K.M. Ho, Strong correlations of dynamical and structural heterogeneities with localized soft modes in a Cu-Zr metallic glass, Applied Physics Letters, 105 (2014) 151910.

[154] H. Wagner, D. Bedorf, S. Küchemann, M. Schwabe, B. Zhang, W. Arnold, K. Samwer, Local elastic properties of a metallic glass, Nature materials, 10 (2011) 439442.

[155] M.J. Demkowicz, A.S. Argon, High-density liquidlike component facilitates plastic flow in a model amorphous silicon system, Physical review letters, 93 (2004) 025505. 
[156] J. Ye, J. Lu, C. Liu, Q. Wang, Y. Yang, Atomistic free-volume zones and inelastic deformation of metallic glasses, Nature materials, 9 (2010) 619-623.

[157] L. Huo, J. Ma, H. Ke, H. Bai, D. Zhao, W. Wang, The deformation units in metallic glasses revealed by stress-induced localized glass transition, Journal of Applied Physics, 111 (2012) 113522.

[158] T. Ichitsubo, E. Matsubara, T. Yamamoto, H. Chen, N. Nishiyama, J. Saida, K. Anazawa, Microstructure of fragile metallic glasses inferred from ultrasound-accelerated crystallization in Pd-based metallic glasses, Physical review letters, 95 (2005) 245501. [159] Y. Hu, F. Li, M. Li, H. Bai, W. Wang, Five-fold symmetry as indicator of dynamic arrest in metallic glass-forming liquids, Nature communications, 6 (2015).

[160] D. Kim, W. Kim, E. Park, N. Mattern, J. Eckert, Phase separation in metallic glasses, Progress in materials science, 58 (2013) 1103-1172.

[161] C. Tang, C.H. Wong, Effect of atomic-level stresses on local dynamic and mechanical properties in $\mathrm{Cu}_{\mathrm{x}} \mathrm{Zr}_{100-\mathrm{x}}$ metallic glasses: A molecular dynamics study, Intermetallics, 58 (2015) 50-55.

[162] Q.K. Li, M. Li, Free volume evolution in metallic glasses subjected to mechanical deformation, Materials transactions, 48 (2007) 1816-1821.

[163] A. Hirata, P. Guan, T. Fujita, Y. Hirotsu, A. Inoue, A.R. Yavari, T. Sakurai, M. Chen, Direct observation of local atomic order in a metallic glass, Nature materials, 10 (2011) 28-33.

[164] A. Zhu, G.J. Shiflet, D.B. Miracle, Glass forming ranges of Al-rare earth metal alloys: thermodynamic and kinetic analysis, Scripta materialia, 50 (2004) 987-991.

[165] J. Ding, Y.-Q. Cheng, H. Sheng, E. Ma, Short-range structural signature of excess specific heat and fragility of metallic-glass-forming supercooled liquids, Physical Review B, 85 (2012) 060201. 
[166] S. Zhao, J. Li, B. Liu, Chemical and topological short-range orders in the ternary $\mathrm{Ni}-\mathrm{Zr}-\mathrm{Al}$ metallic glasses studied by Monte Carlo simulations, Journal of Physics: Condensed Matter, 25 (2013) 095005.

[167] T. Fujita, P.F. Guan, H.W. Sheng, A. Inoue, T. Sakurai, M.W. Chen, Coupling between chemical and dynamic heterogeneities in a multicomponent bulk metallic glass, Physical Review B, 81 (2010) 140204.

[168] S. Chen, H. Zhang, I. Todd, Phase-separation-enhanced plasticity in a

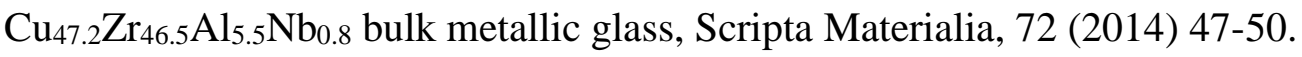

[169] D.-S. Sung, O.-J. Kwon, E. Fleury, K.-B. Kim, J.-C. Lee, D.-H. Kim, Y.-C. Kim, Enhancement of the glass forming ability of $\mathrm{Cu}-\mathrm{Zr}-\mathrm{Al}$ alloys by $\mathrm{Ag}$ addition, Metals and materials International, 10 (2004) 575-579.

[170] E. Park, D. Kim, T. Ohkubo, K. Hono, Enhancement of glass forming ability and plasticity by addition of $\mathrm{Nb}$ in $\mathrm{Cu}-\mathrm{Ti}-\mathrm{Zr}-\mathrm{Ni}-\mathrm{Si}$ bulk metallic glasses, Journal of noncrystalline solids, 351 (2005) 1232-1238.

[171] J. Oh, T. Ohkubo, Y. Kim, E. Fleury, K. Hono, Phase separation in $\mathrm{Cu}_{43} \mathrm{Zr}_{43} \mathrm{Al}_{7} \mathrm{Ag}_{7}$ bulk metallic glass, Scripta materialia, 53 (2005) 165-169.

[172] A. Kündig, M. Ohnuma, T. Ohkubo, T. Abe, K. Hono, Glass formation and phase separation in the Ag-Cu-Zr system, Scripta materialia, 55 (2006) 449-452.

[173] B.E. Warren, X-ray Diffraction, Courier Corporation, 1969.

[174] W. Zhang, F. Jia, Q.s. Zhang, A. Inoue, Effects of additional Ag on the thermal stability and glass-forming ability of $\mathrm{Cu}-\mathrm{Zr}$ binary glassy alloys, Materials Science and Engineering: A, 459 (2007) 330-336.

[175] K. Song, P. Gargarella, S. Pauly, G. Ma, U. Kühn, J. Eckert, Correlation between glass-forming ability, thermal stability, and crystallization kinetics of $\mathrm{Cu}-\mathrm{Zr}$ - $\mathrm{Ag}$ metallic glasses, Journal of Applied Physics, 112 (2012) 063503. 
[176] C.P. Kim, Y.S. Oh, S. Lee, N.J. Kim, Realization of high tensile ductility in a bulk metallic glass composite by the utilization of deformation-induced martensitic transformation, Scripta Materialia, 65 (2011) 304-307.

[177] Z. Liu, R. Li, G. Liu, K. Song, S. Pauly, T. Zhang, J. Eckert, Pronounced ductility in $\mathrm{CuZrAl}$ ternary bulk metallic glass composites with optimized microstructure through melt adjustment, AIP Advances, 2 (2012) 032176.

[178] J. Das, M.B. Tang, K.B. Kim, R. Theissmann, F. Baier, W.H. Wang, J. Eckert, "Work-hardenable" ductile bulk metallic glass, Physical Review Letters, 94 (2005) 205501.

[179] K. Song, S. Pauly, Y. Zhang, R. Li, S. Gorantla, N. Narayanan, U. Kühn, T. Gemming, J. Eckert, Triple yielding and deformation mechanisms in metastable $\mathrm{Cu}_{47.5} \mathrm{Zr}_{47.5} \mathrm{Al}_{5}$ composites, Acta Materialia, 60 (2012) 6000-6012.

[180] F.-F. Wu, K. Chan, S.-S. Jiang, S.-H. Chen, G. Wang, Bulk metallic glass composite with good tensile ductility, high strength and large elastic strain limit, Scientific reports, 4 (2014) 5302.

[181] S.H. Hong, J.T. Kim, H.J. Park, J.Y. Suh, K.R. Lim, Y.S. Na, J.M. Park, K.B. Kim, Work-hardening and plastic deformation behavior of Ti-based bulk metallic glass composites with bimodal sized B2 particles, Intermetallics, 62 (2015) 36-42.

[182] B.J. Kim, W.T. Kim, Researches on the Enhancement of Plasticity of Bulk Metallic Glass Alloys, Applied Microscopy, 45 (2015) 52-57.

[183] K. Albe, Y. Ritter, D. Şopu, Enhancing the plasticity of metallic glasses: Shear band formation, nanocomposites and nanoglasses investigated by molecular dynamics simulations, Mechanics of Materials, 67 (2013) 94-103. 
[184] Y. Wang, G. Hao, Y. Zhang, J. Lin, L. Song, J. Qiao, The role of the interface in a $\mathrm{Ti}$ - based metallic glass matrix composite with in situ dendrite reinforcement, Surface and Interface Analysis, 46 (2014) 293-296. 